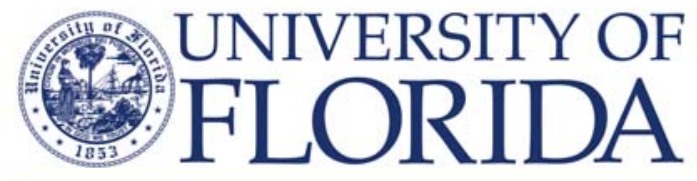

$\mathrm{OH}-94$

IFAS EXTENSION

\title{
Preemergence Herbicides for Use In Ornamentals ${ }^{1}$
}

\author{
Robert H. Stamps, Diane K. Rock and Jeffrey G. Norcini ${ }^{2}$
}

Preemergence herbicides, by definition, are herbicides that are applied prior to weed seed germination. Control of weeds using preemergence herbicides is most successful when the correct herbicide is applied in the correct manner to a weed-free growing medium prior to weed seed germination. In field or landscape situations, the soil should be freshly tilled, and large clods of soil broken up. The growing medium, whether soil or soilless, should be settled and firm at the time of herbicide application.

Herbicides should be applied uniformly to the target area and then immediately incorporated into the growing medium. Uniform herbicide coverage is dependent on good application technique and, for mechanized application, on well-maintained and calibrated equipment. Incorporation is generally employed to reduce herbicide losses from volatility and photodecomposition, but it also serves to activate some herbicides. It is commonly accomplished by applying water using overhead irrigation, often about 0.5 inch [1.2 cm], after the herbicide has been applied. Herbicides can also be mechanically incorporated by mixing the herbicide into the top layer of the growing medium, generally to a depth of 1-3 inches [2.5-7.5 cm]. Recommendations regarding the depth of mechanical incorporation and/or the amount of irrigation water to apply to activate/incorporate the herbicides should be followed carefully in order to minimize the possibility of crop injury.

1. This is document $\mathrm{OH}-94$, one of a series of the Department of Environmental Horticulture, Florida Cooperative Extension Service, Institute of Food and Agricultural Sciences, University of Florida. First published March 1993. Revised November 2005. Please visit the EDIS Web site at http://edis.ifas.ufl.edu.

2. Robert H. Stamps and Diane R. Rock, Professor and Research Assistant, respectively, Mid-Florida Research and Education Center-Apopka; and Jeffrey G. Norcini, Assoc. Professor, North Florida Research and Education Center-Quincy.

Mention of a commercial or proprietary product or chemical does not constitute a recommendation or warranty of the product by the authors or the University of Florida, Institute of Food and Agricultural Sciences, nor does it imply its approval to the exclusion of other products that may also be suitable. Products should be used according to label instructions and safety equipment required on the label and by federal or state law should be employed. Users should avoid the use of chemicals under conditions that could lead to ground water contamination. Pesticide registrations may change so it is the responsibility of the user to ascertain if a pesticide is registered by the appropriate state and federal agencies for an intended use.

The Institute of Food and Agricultural Sciences (IFAS) is an Equal Employment Opportunity - Affirmative Action

Employer authorized to provide research, educational information and other services only to individuals and institutions that function without regard to race, color, sex, age, disability or national origin. U.S. Department of Agriculture,

Cooperative Extension Service, University of Florida, IFAS, Florida A. \& M. University Cooperative Extension Program, and Boards of County Commissioners Cooperating. 
Many of the preemergence herbicides used in ornamentals are formulated as granular materials and should be applied evenly over the treated area. Avoid applications to moist foliage or to whorled or other foliage types that will accumulate and trap the granules. Application equipment used to broadcast these formulations should not grind the granules or increase the amount of dust; this may generate potentially dangerous conditions to nearby plants and people. Use materials that have been screened of fine particles, if available.

For liquid applications, spray pressure should be constant and adequate to maintain proper spray pattern for uniformity and ensure droplet penetration through the plant canopy. Excessive pressure can lead to drift and damage to non-target plants. Spray volume should be sufficient for thorough coverage. Herbicides should be thoroughly mixed (agitated) in spray tanks to obtain uniform results, and spray nozzle openings (orifices) should be checked regularly and replaced when wear becomes evident. Spray adjuvants that enhance coverage, penetration and/or persistence of herbicides can be added to some spray mixtures, especially if the mixtures are applied so that the crop foliage is not treated. Test spray solutions that contain any new adjuvant for phytotoxicity on a small part of your crop, just as you would test any new pesticide.

Chemigation is a special type of liquid application in which herbicides are applied using the irrigation equipment. It is the least labor-intensive method to apply herbicides; however, few herbicides are labeled for application in this manner (see Table 1). In addition, some types of irrigation systems (e.g., drip) do not apply water to all areas that need to be treated and/or do not have adequate uniformity for this use. Herbicide formulations applied through irrigation systems must not clog nozzle orifices. Florida and other states have laws that require that safety equipment must be provided if chemicals are to be injected into irrigation systems. Some of the equipment necessary to prevent contamination of the water supply includes check valves, vacuum breakers, low-pressure drains, shutoff valves, remote chemical storage tanks and interconnected power supplies to injector and irrigation pumps.

Liquid herbicides should be measured volumetrically, i.e., using measuring cups, graduated cylinders, etc. Since dry pesticides vary in density, it is not possible to give accurate volumetric conversions across brands and formulations so those formulations must be weighed.

It is a good practice to keep records of all pesticide applications, even of nonrestricted use pesticides - including EPA registration and product lot numbers. These records can be useful for planning future weed control measures. In addition, they can be invaluable if crop damage occurs.

Regardless of the herbicide or application method used, it is strongly recommended that the effects should be evaluated against untreated controls under your particular conditions before treating large areas. This is especially important when there is a statement on the label permitting use of the product on an ornamental not specifically listed on the label. Treat a limited area or number of plants and wait 2 to 3 weeks, or longer if the label recommends, for any phytotoxic effects to appear. Be aware that the larger the area treated, the more likely that phytotoxicity may occur, especially due to volatilization. Also, damage may not occur the first time a herbicide is applied, but may 
show up with repeated applications. Several formulations of a herbicide may be available with varying concentrations; consequently, recommendations on the manufacturer's label should be followed explicitly. These herbicides formulations are labeled for application in certain environments (outdoors, in shadehouses and/or in greenhouses) and specific locations (containers, field and/or landscape) and can only be applied in those specified environments and locations.

Although most preemergence herbicides labeled for use in ornamentals have relatively low acute mammalian toxicities, they are potentially dangerous if handled improperly and, therefore, the safety precautions on the label(s) must be followed. Read the entire label, including the small print, before buying or using the herbicide. Wear personal protective equipment (PPE) and comply with restricted entry intervals (REI) (see Table 1). Keep the telephone number and address of the nearest County Poison Control Center listed in a convenient location in case of an accidental poisoning. Have clean copies of herbicide labels and material safety data sheets (MSDS) available to be taken to the Poison Control Center or hospital in the event of an emergency. If a herbicide is labeled for use specifically in a particular state [for example, 24(c) Special Local Need labeling in Florida], obtain a copy of the supplemental label from your supplier when you purchase the product; otherwise, you will be using it illegally.

Many of these herbicides have low solubilities in water and are quite strongly held to binding sites in growing media and are, therefore, not readily leachable. However, the relatively long persistence (long degradation half-lives, $\mathrm{T}_{1 / 2}$ ) of some of these products creates the potential for leaching losses to still occur with potential effects on ground water. Relative leaching potential indexes (RLPI) for the listed products range from 3 (high potential to leach) to $>2,000$ (low potential for leaching, very immobile) and are given, along with relative run-off potential indexes (RRPI), in Table 1. RRPIs range from 1 (high potential to be lost in runoff) to 333 (lower run-off potential). For more information about pesticide leaching and run-off, read Managing Pesticides for Ornamentals Production and Water Quality Protection (University of Florida, Institute of Food and Agricultural Sciences, Cooperative Extension Service, Soil Science Department Circular 991, available at http://edis.ifas.ufl.edu/).

As an aid to help delay the selection of herbicide-resistant weeds, the herbicide group to which each product belongs is listed in Table 1. The groups are based according to primary sites/modes of action (Weed Technology 11:384-393 [1997]) and can be used to select herbicides that have differing sites/modes of action. Repeated use of a herbicide or herbicides having the same sites of action can, over time, cause development of weeds resistant to those herbicides.

Store herbicides in their original labeled containers, out of reach of children. All pesticides should be locked in secure facilities. When containers are empty, rinse with water at least three times and pour the rinse water into the spray tank. Dispose of empty containers promptly and safely (according to local, state and federal disposal laws).

In Table 2, weeds are listed alphabetically by scientific name and along with the preemergence herbicides that control $(\mathrm{C})$, suppress $(\mathrm{S})$ or do not control $(\mathrm{N})$ each weed. Table 
3 is similar except that the weeds are listed alphabetically by common name(s). The information in these tables is derived from product labels, and published and unpublished research results. A herbicide may provide variable weed control depending on soil, irrigation and other variables. Where the weed entry in the tables lists only a genus, the labels must be checked to see which species in that genus are controlled by each herbicide.

Table 4 lists the tolerances of ornamentals to preemergence herbicides, with the table subdivided into Trees and Shrubs, Groundcovers and Vining Plants, and Annuals and Perennials. The plants are listed alphabetically by scientific name. This information is derived from product labels. It is only presented as a guide and does not imply that a herbicide can be used in or around that ornamental species in all sites (nursery, landscape, greenhouse). In Table 4, Y indicates that the crop is on product label and $\mathrm{N}$ indicates that the product should not be used on the crop. Please note that not all plant species and cultivars listed on the herbicide labels are included in the table and some plants are listed in the table even though, at the current time, they are not listed on any of the herbicide labels. Hopefully, research and future label changes will help alter this situation for this latter group of plants.

To help in situations where the reader does not know the scientific name of the crop, Table 5 is included. It lists the common names of crops beside their scientific names; however, readers should be aware that the same common name may be used for more than one plant so the true identity of the crop should be ascertained before herbicides are applied.

When selecting a herbicide, both of the factors listed below must be determined to legally use a herbicide in or around an ornamental species; the information below is on the product label:

1. Determine the tolerances of specific species, varieties, or cultivars, if applicable,

AND

2. Determine which sites the herbicide can be used on, that is - nursery (container or field); landscape (commercial, public, or residential); enclosed greenhouse.

Please report any errors, omissions and/or updates to Bob Stamps

(rhs@ifas.ufl.edu).

Thank you. 
Table 1. Trade names, active ingredients, manufacturers/distributors, labeling for chemigation, restricted-entry intervals (REI), vapor pressure (volatility), relative leaching and relative run-off potential indexes (RLPI, RRPI), and herbicide group (mode of action) for some preemergence herbicides labeled for ornamental crops. Various fertilizer formulations containing preemergence herbicides are also available but are not listed in this table. Be aware that not all these products are labeled for use on containerized ornamentals or in all locations (greenhouse, landscape, nursery).

\begin{tabular}{|c|c|c|c|c|c|c|c|c|}
\hline Trade name & $\begin{array}{l}\text { Common name } \\
\text { (active ingredient) }\end{array}$ & $\begin{array}{l}\text { Manufacturer or } \\
\text { distributor }\end{array}$ & $\begin{array}{l}\text { Labeled for } \\
\text { chemigation }\end{array}$ & $\begin{array}{c}\text { REI } \\
\text { (hours) }\end{array}$ & $\begin{array}{l}\text { Vapor pressure } \\
(\mathrm{mPa})^{1}\end{array}$ & $\mathrm{RLPI}^{2}$ & $\mathrm{RRPI}^{2}$ & $\begin{array}{l}\text { Herbicide } \\
\text { group }^{3}\end{array}$ \\
\hline Barricade $^{\circledR} 4 \mathrm{FL}, 65 \mathrm{WG}$ & prodiamine & Syngenta & $\mathrm{NO}$ & 12 & 0.003 & 1,080 & 1 & 3 \\
\hline Barrier $^{\circledR} 4 \mathrm{G}$ & dichlobenil & PBI/Gordon & $n a^{4}$ & $\mathrm{nd}^{5}$ & 135.00 & 67 & 42 & 20 \\
\hline Bensumec $^{\mathrm{TM}} 4 \mathrm{LF}$ & bensulide & PBI/Gordon & $\mathrm{NO}$ & when dry & 0.10 & 83 & 8 & 8 \\
\hline BroadStar $^{\mathrm{TM}} 0.25 \mathrm{G}$ & flumioxazin & Valent USA & na & 12 & 0.32 & 130 & 130 & 14 \\
\hline Casoron $^{\circledR} 4 \mathrm{G}$ & dichlobenil & Uniroyal & na & 12 & 135.00 & 67 & 42 & 20 \\
\hline Cavalcade $^{\mathrm{TM}}$ 65WDG & prodiamine & Sipcam Agro USA & $\mathrm{NO}$ & 12 & 0.003 & 1,080 & 1 & 3 \\
\hline Corral $^{\text {TM }} 2.68 \mathrm{G}$ & pendimethalin & Scotts & na & 12 & 3.99 & 556 & 2 & 3 \\
\hline Dacthal $^{\circledR} 6 \mathrm{~F}, 75 \mathrm{WP}$ & DCPA & AMVAC & YES & 12 & 0.33 & 500 & 2 & 3 \\
\hline Devrinol $^{\circledast} 50 \mathrm{DF}$ & napropamide & United Phosphorus & $\mathrm{NO}$ & 12 & 0.53 & 100 & 20 & 15 \\
\hline Devrinol $^{\circledR} 2 \mathrm{G}$ & napropamide & United Phosphorus & na & 12 & 0.53 & 100 & 20 & 15 \\
\hline $\begin{array}{l}\text { Dimension }{ }^{\circledR} 1 \mathrm{EC}, 2 \mathrm{SC}, \\
40 \mathrm{WSP}\end{array}$ & dithiopyr & Dow AgroSciences & $\mathrm{NO}$ & 12 & 0.53 & 410 & 15 & 3 \\
\hline Eptam $^{\circledR} 7 \mathrm{E}$ & EPTC & Gowan & YES & 12 & 4,700 & 333 & 333 & 8 \\
\hline Galigan $^{\circledR} 2 \mathrm{EC}$ & oxyfluorfen & Makhteshim Agan & $\mathrm{NO}^{6}$ & 24 & 0.27 & $>2,000$ & 1 & 14 \\
\hline Gallery $^{\mathrm{TM}}$ 75DF & isoxaben & Dow AgroSciences & $\mathrm{NO}$ & 12 & 0.05 & 140 & 7 & 21 \\
\hline Goal $^{\circledR} 1.6 \mathrm{E}, 2 \mathrm{EC}$ & oxyfluorfen & Dow AgroSciences & $\mathrm{NO}^{6}$ & 24 & 0.27 & $>2,000$ & 1 & 14 \\
\hline Hurdle $^{\circledR} 3.8$ ACS & pendimethalin & Olympic Horticultural & $\mathrm{NO}$ & 24 & 3.99 & 556 & 2 & 3 \\
\hline Image $^{\circledR} 70 \mathrm{DG}$ & imazaquin & BASF & $\mathrm{NO}$ & 12 & 0.003 & 3 & 3 & 2 \\
\hline $\mathrm{Kerb}^{\circledR} 50 \mathrm{WP}^{7}$ & pronamide & Dow AgroSciences & $\mathrm{NO}$ & 24 & 11.31 & 133 & 20 & 15 \\
\hline Lasar $^{\text {TM }} 3 \mathrm{G}$ & oxyfluorfen + oxadiazon & Verdicon & na & 24 & $0.27+0.10$ & 533 & 1 & $14+14$ \\
\hline Lasso $^{\circledR} 4 \mathrm{EC}^{8}$ & alachlor & Monsanto & $\mathrm{NO}$ & 12 & 2.93 & 113 & 113 & 15 \\
\hline $\mathrm{OH} 2^{\circledR} 3 \mathrm{G}$ & oxyfluorfen + pendimethalin & Scotts & na & 24 & $0.27+3.99$ & 556 & 1 & $14+3$ \\
\hline OO-Herbicide ${ }^{\mathrm{TM}}$ 3G & oxyfluorfen + oxadiazon & Regal Chemical & na & 24 & $0.27+0.10$ & 533 & 1 & $14+14$ \\
\hline OxiFlo 2EC & oxyfluorfen & FarmSaver.com & $\mathrm{NO}^{6}$ & 24 & 0.27 & $>2,000$ & 1 & 14 \\
\hline
\end{tabular}

Preemergence Herbicides for Use in Ornamentals 
Table 1. Trade names, active ingredients, manufacturers/distributors, labeling for chemigation, restricted-entry intervals (REI), vapor pressure (volatility), relative leaching and relative run-off potential indexes (RLPI, RRPI), and herbicide group (mode of action) for some preemergence herbicides labeled for ornamental crops. Various fertilizer formulations containing preemergence herbicides are also available but are not listed in this table. Be aware that not all these products are labeled for use on containerized ornamentals or in all locations (greenhouse, landscape, nursery).

\begin{tabular}{|c|c|c|c|c|c|c|c|c|}
\hline Trade name & $\begin{array}{l}\text { Common name } \\
\text { (active ingredient) }\end{array}$ & $\begin{array}{l}\text { Manufacturer or } \\
\text { distributor }\end{array}$ & $\begin{array}{l}\text { Labeled for } \\
\text { chemigation }\end{array}$ & $\begin{array}{c}\text { REI } \\
\text { (hours) }\end{array}$ & $\begin{array}{l}\text { Vapor pressure } \\
\quad(\mathrm{mPa})^{1}\end{array}$ & $\mathrm{RLPI}^{2}$ & $\mathrm{RRPI}^{2}$ & $\begin{array}{l}\text { Herbicide } \\
\text { group }^{3}\end{array}$ \\
\hline Pendulum ${ }^{\circledR} 2 \mathrm{G}$ & pendimethalin & BASF & na & 24 & 3.99 & 556 & 2 & 3 \\
\hline $\begin{array}{l}\text { Pendulum }{ }^{\circledR} 3.8 \mathrm{CS}, 3.3 \mathrm{EC} \text {, } \\
60 \mathrm{WDG}\end{array}$ & pendimethalin & BASF & NO & 24 & 3.99 & 556 & 2 & 3 \\
\hline Pennant Magnum ${ }^{\circledR} 7.6 \mathrm{EC}$ & S-metolachlor & Syngenta & YES & 24 & 4.2 & 22 & 22 & 15 \\
\hline Predict $^{\circledR} 78.6 \mathrm{WDG}$ & norflurazon & Nufarm Turf \& Specialty & $\mathrm{NO}$ & 12 & 0.004 & 233 & 47 & 12 \\
\hline Preen $^{\circledR} 1.875 G$ & trifluralin + isoxaben & Lebanon Seaboard & na & 12 & $14.63+0.05$ & 140 & 2 & $3+21$ \\
\hline Prefar $^{\circledR} 4 \mathrm{E}$ & bensulide & Gowan & YES & 12 & 0.10 & 83 & 8 & 8 \\
\hline Pre- $\mathrm{M}^{\circledR} 3.3 \mathrm{EC}$ & pendimethalin & Lesco & $\mathrm{NO}$ & 24 & 3.99 & 556 & 2 & 3 \\
\hline Pre-M ${ }^{\circledR}$ 60DG & pendimethalin & Lesco & $\mathrm{NO}$ & na & 3.99 & 556 & 2 & 3 \\
\hline PrePair $^{\circledast}$ 6G & napropamide + oxadiazon & Verdicon & na & 12 & 0.53 & 100 & 5 & $15+14$ \\
\hline Pre-San ${ }^{\circledR} 7 G, 12.5 G$ & bensulide & PBI/Gordon & na & when dry & 0.10 & 83 & 8 & 8 \\
\hline Princep $^{\circledR} 4 \mathrm{~L}$ & simazine & Syngenta & NO & 12 & 0.003 & 22 & 22 & 5 \\
\hline ProClipse $^{\mathrm{TM}}$ & prodiamine & Nufarm Turf \& Specialty & NO & 12 & 0.003 & 1,080 & 1 & 3 \\
\hline ProdiaGuard 65WDG & prodiamine & Control Solutions & YES & 12 & 0.003 & 1,080 & 1 & 3 \\
\hline RegalKade $^{\circledR} 0.5 \mathrm{G}, 65 \mathrm{WDG}$ & prodiamine & Regal Chemical & na, NO & 12 & 0.003 & 1,080 & 1 & 3 \\
\hline RegalStar ${ }^{\circledR} \mathrm{G} \&$ II, $1.2 \mathrm{G}$ & oxadiazon + prodiamine & Regal Chemical & na & 12 & $0.10+0.003$ & 533 & 1 & $14+3$ \\
\hline Ronstar $^{\circledR} 2 \mathrm{G}$ & oxadiazon & Bayer CropScience, Regal & na & 12 & 0.10 & 533 & 5 & 14 \\
\hline Ronstar $^{\circledR} 50$ WSP & oxadiazon & Bayer CropScience & $\mathrm{NO}$ & 12 & 0.10 & 533 & 5 & 14 \\
\hline Rout $^{\circledR} 3 G$ & oxyfluorfen + oryzalin & Scotts & na & 24 & $0.27+<0.001$ & 300 & 1 & $14+3$ \\
\hline Showcase $^{\mathrm{TM}} 2.5 \mathrm{G}$ & $\begin{array}{l}\text { trifluralin }+ \text { isoxaben }+ \\
\text { oxyfluorfen }\end{array}$ & Dow AgroSciences & na & 24 & $\begin{array}{c}14.63+0.05+ \\
0.27\end{array}$ & 140 & 1 & $3+21+14$ \\
\hline Simazine 4L & simazine & Drexel, Riverside, UAP & YES & 12 & 0.003 & 22 & 22 & 5 \\
\hline Simazine 90DF, 90WDG & simazine & Drexel, Riverside, UAP & NO & 12 & 0.003 & 22 & 22 & 5 \\
\hline Sim-Trol $^{\circledR} 4 \mathrm{~L}, 90 \mathrm{DF}$ & simazine & Sipcam Agro USA & $\mathrm{NO}$ & 12 & 0.003 & 22 & 22 & 5 \\
\hline
\end{tabular}

Preemergence Herbicides for Use in Ornamentals 
Table 1. Trade names, active ingredients, manufacturers/distributors, labeling for chemigation, restricted-entry intervals (REI), vapor pressure (volatility), relative leaching and relative run-off potential indexes (RLPI, RRPI), and herbicide group (mode of action) for some preemergence herbicides labeled for ornamental crops. Various fertilizer formulations containing preemergence herbicides are also available but are not listed in this table. Be aware that not all these products are labeled for use on containerized ornamentals or in all locations (greenhouse, landscape, nursery).

\begin{tabular}{|c|c|c|c|c|c|c|c|c|}
\hline Trade name & $\begin{array}{l}\text { Common name } \\
\text { (active ingredient) }\end{array}$ & $\begin{array}{l}\text { Manufacturer or } \\
\text { distributor }\end{array}$ & $\begin{array}{l}\text { Labeled for } \\
\text { chemigation }\end{array}$ & $\begin{array}{c}\text { REI } \\
\text { (hours) }\end{array}$ & $\begin{array}{c}\text { Vapor pressure } \\
(\mathrm{mPa})^{1}\end{array}$ & RLPI $^{2}$ & $\mathrm{RRPI}^{2}$ & $\begin{array}{l}\text { Herbicide } \\
\text { group }^{3}\end{array}$ \\
\hline Snapshot $^{\circledR}$ 2.5TG & trifluralin + isoxaben & Dow AgroSciences & na & 12 & $14.63+0.05$ & 140 & 2 & $3+21$ \\
\hline SureGuard $^{\mathrm{TM}}$ 51WDG & flumioxazin & Valent USA & na & 12 & 0.32 & 130 & 130 & 14 \\
\hline Surflan $^{\circledR} 1.67 \mathrm{G}$ & oryzalin & Verdicon & $\mathrm{NO}$ & $24^{9}$ & $<0.001$ & 300 & 83 & 3 \\
\hline Surflan ${ }^{\circledR} 4$ AS Specialty & oryzalin & $\begin{array}{l}\text { United Phosphorus, } \\
\text { Verdicon }\end{array}$ & $\mathrm{NO}$ & $24^{9}$ & $<0.001$ & 300 & 83 & 3 \\
\hline Treflan $^{\text {TM }} 5 \mathrm{G}$ & trifluralin & $\begin{array}{l}\text { Lebanon Seaboard, } \\
\text { Verdicon }\end{array}$ & na & 12 & 14.63 & 1330 & 2 & 3 \\
\hline Weedfree $63^{\mathrm{TM}} 2 \mathrm{G}$ & oxyfluorfen & Harrell's & na & 24 & 0.27 & $>2,000$ & 1 & 14 \\
\hline Weedfree $75^{\mathrm{TM}} 5 \mathrm{G}$ & oxyfluorfen + trifluralin & Harrell's & na & 24 & $0.27+14.63$ & 1330 & 1 & $14+3$ \\
\hline $\mathrm{XL}^{\circledR} 2 \mathrm{G}$ & benefin + oryzalin & Helena Chemical & na & 24 & $10.37+<0.001$ & 300 & 3 & $3+3$ \\
\hline
\end{tabular}

${ }^{1}$ Vapor pressure at $25^{\circ} \mathrm{C}\left(77^{\circ} \mathrm{F}\right)$.

${ }^{2}$ RLPI = relative leaching potential index, RRPI=relative runoff potential index. The higher the number, the less likely for herbicide movement by that mechanism. See Managing Pesticides for Ornamentals Production and Water Quality Protection (University of Florida, Institute of Food and Agricultural Sciences, Cooperative Extension Service, Soil Science Department Circular 991, available at http://edis.ifas.ufl.edu) for more details on these indexes.

${ }^{3}$ Herbicide groups are based according to primary sites of action (Weed Technology 11:384-393, [1997]) and can be used to select herbicides that have differing sites of action. This information can be used to help select herbicides so as to minimize the potential for the development of herbicide-resistant weeds. $2=\mathrm{ALS} / \mathrm{AHAS}$ inhibitors, $3=$ microtubule assembly inhibitors, $5=$ photosystem II inhibitors, $8=$ lipid synthesis inhibitors, $12=$ carotenoid biosynthesis inhibitors, $14=$ PPO inhibitors, $15=$ acetamides (unknown site of action), $20=$ cellulose synthase inhibitors, 21 = inhibition of cell wall synthesis site B.

${ }^{4}$ na $=$ not applicable.

${ }^{5} \mathrm{nd}=$ no data.

${ }^{6}$ Except conifer seedbeds irrigated with center pivot sprinkler irrigation.

${ }^{7} \mathrm{Kerb}^{\circledR}$ is a restricted use pesticide.

${ }^{8}$ Lasso $^{\circledast}$ is not labeled for use in certain locations; for example, Florida and Nassau and Suffolk counties in New York. Before using Lasso ${ }^{\circledast}$, check to make sure it is registered for use in your area.

${ }^{9}$ Workers may enter treated areas without required PPE during the reentry interval following $1 / 2$ to 1 inch of rainfall or irrigation, if they are performing tasks that do not involve contact with the soil subsurface. 


\begin{tabular}{|c|c|c|c|c|c|c|c|c|c|c|c|c|c|c|c|c|c|c|c|c|c|c|c|c|c|c|c|c|c|c|}
\hline Weed scientific name & 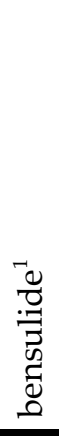 & 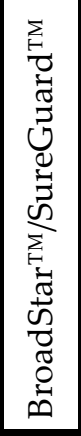 & 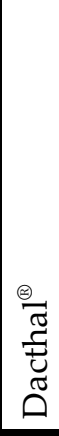 & 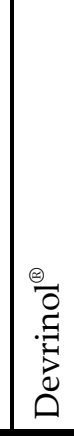 & 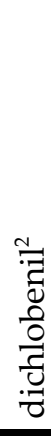 & 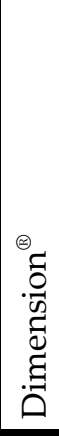 & 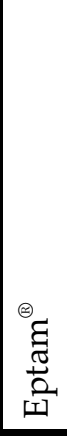 & 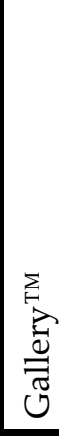 & \begin{tabular}{|l|}
$\Theta_{0}$ \\
$\infty$ \\
$\widetilde{\Xi}$ \\
$\Xi$ \\
\end{tabular} & 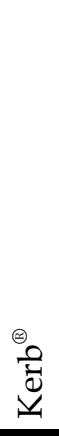 & 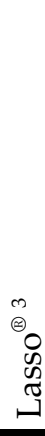 & $\stackrel{\oplus}{\stackrel{\oplus}{T}}$ & 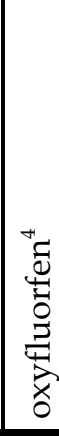 & |r & 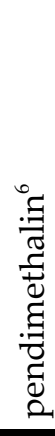 & 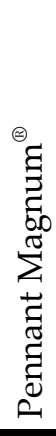 & 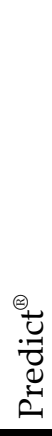 & $\frac{\Theta}{\nexists Z}$ & 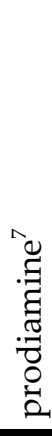 & 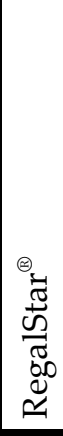 & 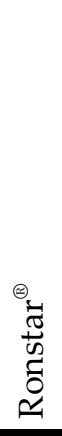 & 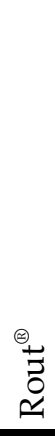 & $\begin{array}{l}\text { o } \\
\text { J } \\
3 \\
0 \\
\text { ஸे }\end{array}$ & 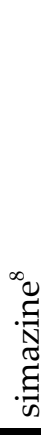 & \% & E & & & 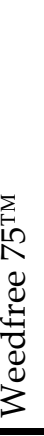 & $\stackrel{\oplus}{\times}$ \\
\hline Amaranthus spp. ${ }^{10}$ & $\mathrm{C}$ & $\mathrm{C}$ & $S$ & $\mathrm{C}$ & $\mathrm{C}$ & $\mathrm{C}$ & C & $\mathrm{C}$ & $\mathrm{C}$ & & $\mathrm{C}$ & $\mathrm{C}$ & $\mathrm{C}$ & $\mathrm{C}$ & $\mathrm{C}$ & $\mathrm{C}$ & $\mathrm{C}$ & $\mathrm{C}$ & $\mathrm{C}$ & $\mathrm{C}$ & $\mathrm{C}$ & C & $\mathrm{C}$ & $\mathrm{C}$ & C & 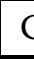 & & & $\mathrm{C}$ & $\mathrm{C}$ \\
\hline Ambrosia artemisiifolia & $\mathrm{N}$ & $S$ & $\mathrm{~N}$ & $\mathrm{~S} / \mathrm{C}$ & C & & & $\mathrm{C}$ & & $\mathrm{C}$ & $\mathrm{S}$ & & $S$ & & $\mathrm{~N}$ & $\mathrm{~S}$ & $\mathrm{~S}$ & C & $\mathrm{S}$ & & $\mathrm{C}$ & & C & C & $\mathrm{S}$ & 1 & & & & $\mathrm{~S}$ \\
\hline Anthemis cotula & & $\mathrm{C}$ & & & & & & $\mathrm{C}$ & & & & & $\mathrm{C}$ & & & & & & & & & & $\mathrm{C}$ & & & & & & $\mathrm{C}$ & \\
\hline Bidens bipinnata & & & & & C & & & & & & & & & & & & & & $\mathrm{N}$ & & & & & C & & & & & & \\
\hline Brassica spp. ${ }^{10}$ & & $-11 / C$ & $\mathrm{~N}$ & $S$ & $\mathrm{C}$ & $\mathrm{C}$ & & $\mathrm{C}$ & & $\mathrm{C}$ & $\mathrm{N}$ & & $\mathrm{C}$ & & & $\mathrm{N}$ & & & & & $\mathrm{C}$ & C & $\mathrm{C}$ & $\mathrm{C}$ & $\mathrm{S}$ & & & & $\mathrm{C}$ & $S$ \\
\hline Bryum spp. & & $\mathrm{C}$ & & & & & & & & & & & & & & & & & & & & $\mathrm{C}$ & & & & & & & & \\
\hline Capsella bursa-pastoris & $\mathrm{C}$ & C & $S$ & $\mathrm{C}$ & C & C & & $\mathrm{C}$ & & $\mathrm{C}$ & & $\mathrm{C}$ & $S / C$ & $\mathrm{C}$ & $\mathrm{C}$ & & $\mathrm{C}$ & C & C & & C & $\mathrm{C}$ & C & C & $\mathrm{C}$ & & & & $\mathrm{C}$ & $\mathrm{C}$ \\
\hline Cardamine spp..$^{10}$ & & $\mathrm{C}$ & $\mathrm{N}$ & $\mathrm{C}$ & $\mathrm{C}$ & $\mathrm{C}$ & & $\mathrm{C}$ & $\mathrm{C}$ & & & $\mathrm{C}$ & $\mathrm{C}$ & $\mathrm{C}$ & $\mathrm{N}$ & $\mathrm{N}$ & & $\mathrm{C}$ & & $\mathrm{C}$ & $\mathrm{C}$ & $\mathrm{C}$ & $\mathrm{C}$ & $\mathrm{S}$ & $\mathrm{C}$ & 1 & & & $\mathrm{C}$ & $\mathrm{C}$ \\
\hline Cerastium vulgatum & & C & & $\mathrm{N}$ & C & & & $\mathrm{C}$ & C & $\mathrm{C}$ & & & & & $\mathrm{C}$ & & & & & & & & $\mathrm{C}$ & $\mathrm{N}$ & $\mathrm{N}$ & & & & & \\
\hline $\begin{array}{l}\text { Chamaesyce hirta (synonym } \\
\text { Euphorbia hirta) }\end{array}$ & $\mathrm{N}$ & C & & & & C & & & & $\mathrm{N}$ & & C & $C^{12}$ & C & & & & C & & & & C & & & & & & & & \\
\hline $\begin{array}{l}\text { C. humistrata, (synonym } \\
\text { Euphorbia humistrata) }\end{array}$ & $\mathrm{N}$ & C & $S$ & $\mathrm{~N}$ & C & C & & C & & $\mathrm{N}$ & & C & & C & C & $\mathrm{S}$ & C & C & C & S & $S$ & C & C & S & C & & & & & $S / C$ \\
\hline $\begin{array}{l}\text { C. maculata, (synonym E. } \\
\text { maculata, E. supina) }\end{array}$ & $\mathrm{N}$ & C & $S$ & $\mathrm{~N}$ & C & C & & $\mathrm{S} / \mathrm{C}$ & & $\mathrm{N}$ & & C & C & C & C & & $\mathrm{S}$ & & & C & $\mathrm{S}$ & C & & $\mathrm{S}$ & S & & & & C & $\mathrm{S}$ \\
\hline $\begin{array}{l}\text { C. vermiculata, (synonym } \\
\text { Euphorbia vermiculata) }\end{array}$ & $\mathrm{N}$ & & & & & & & & & $\mathrm{N}$ & & & & & & & & & & & & C & & & & & & & & \\
\hline Chenopodium spp. ${ }^{10}$ & $\mathrm{C}$ & $\mathrm{C}$ & $\mathrm{C}$ & $\mathrm{C}$ & $\mathrm{C}$ & & $\mathrm{C}$ & $\mathrm{C}$ & & $\mathrm{C}$ & $\mathrm{S}$ & & $\mathrm{C}$ & & C & $S$ & $\mathrm{~S}$ & $\mathrm{C}$ & $\mathrm{C}$ & $\mathrm{C}$ & $\mathrm{C}$ & $\mathrm{C}$ & $\mathrm{C}$ & C & C & ( & & & C & $\mathrm{C}$ \\
\hline Commelina diffusa & & & & & & & & & & & & & & & & & $S$ & & C & & & & & & & & & & & \\
\hline Cyperus compressus & & & & & & & & $\mathrm{N}$ & $\mathrm{S}$ & & & & & & & & $\mathrm{C}$ & C & & & $\mathrm{C}$ & & & & & & & & & \\
\hline Cyperus esculentus & $\mathrm{N}$ & & $\mathrm{N}$ & $S$ & C & & C & $\mathrm{N}$ & $\mathrm{S} / \mathrm{C}$ & $\mathrm{N}$ & $S$ & $\mathrm{~N}$ & $\mathrm{~N}$ & & $\mathrm{~N}$ & C & $\mathrm{S}$ & & $\mathrm{N}$ & $\mathrm{N}$ & $\mathrm{N}$ & $\mathrm{N}$ & & $\bar{N}$ & $\mathrm{~N}$ & 1 & & & & \\
\hline Cyperus globulosus & & & & & & & & & $C$ & & & & & & & & & & & & & & & & & & & & & \\
\hline
\end{tabular}

\section{Preemergence Herbicides for Use in Ornamentals}




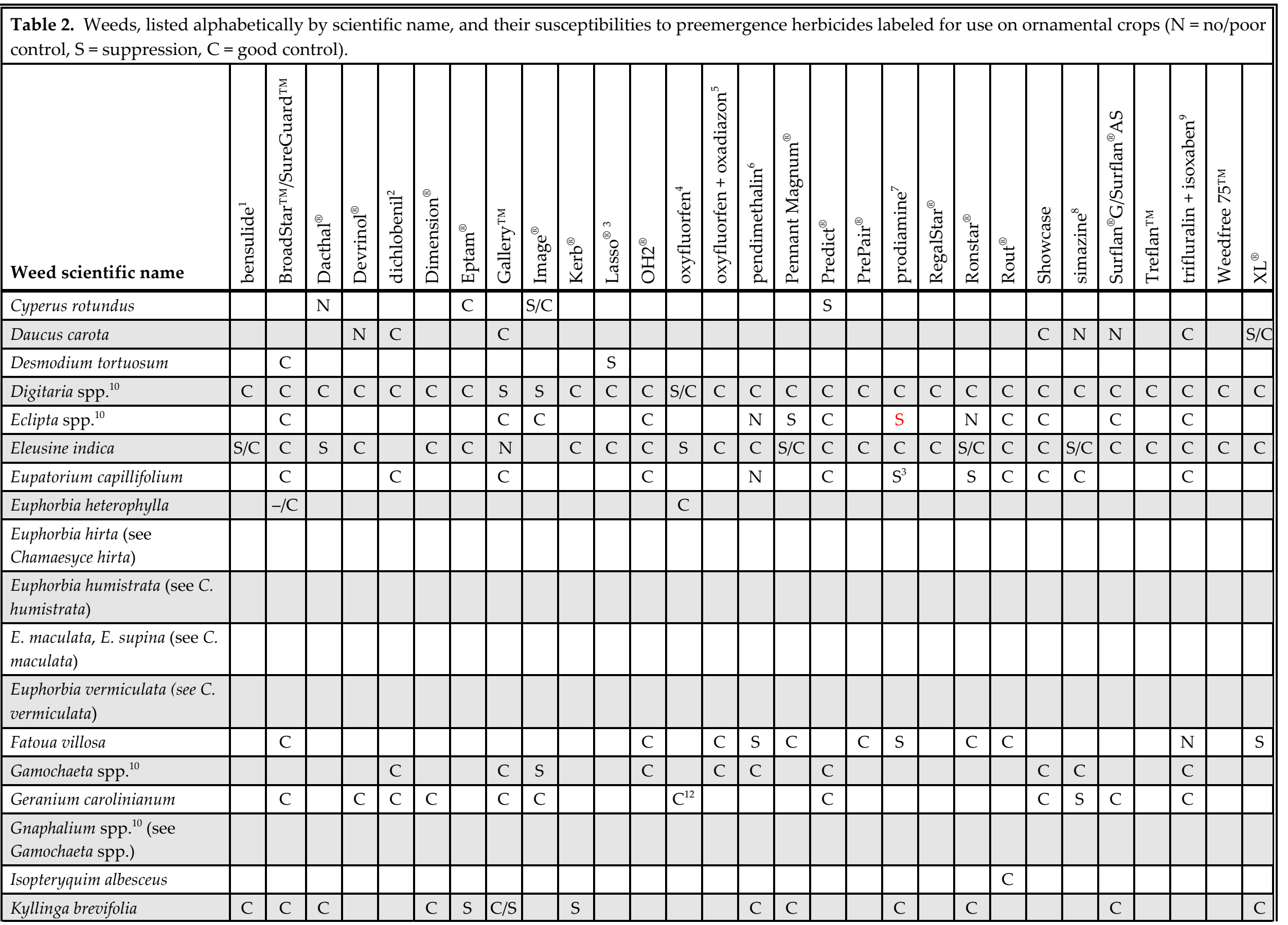

\section{Preemergence Herbicides for Use in Ornamentals}




\begin{tabular}{|c|c|c|c|c|c|c|c|c|c|c|c|c|c|c|c|c|c|c|c|c|c|c|c|c|c|c|c|c|c|}
\hline Weed scientific name & 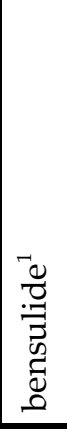 & 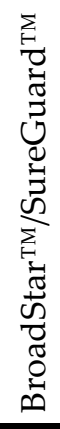 & $\begin{array}{l}\stackrel{\oplus}{\widetilde{\sigma}} \\
\stackrel{\oplus}{\tilde{U}} \\
\tilde{\sigma} \\
0\end{array}$ & 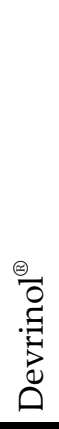 & 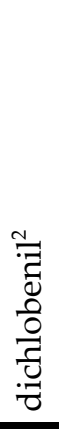 & 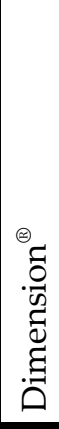 & 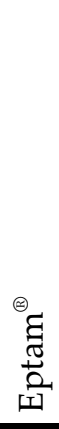 & 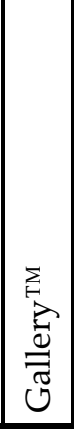 & 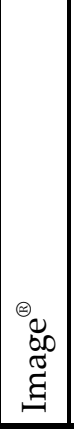 & 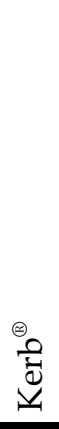 & 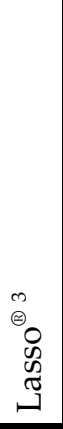 & $\stackrel{\stackrel{\oplus}{N}}{\stackrel{\oplus}{O}}$ & 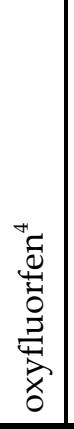 & 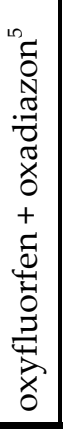 & 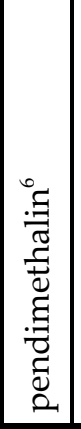 & 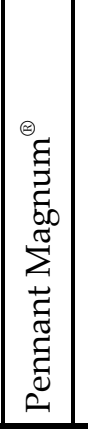 & 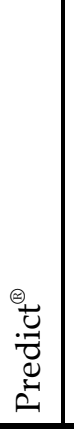 & 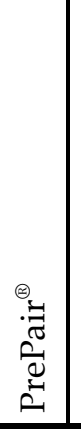 & 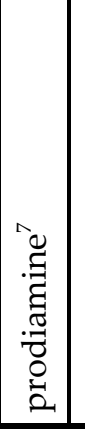 & 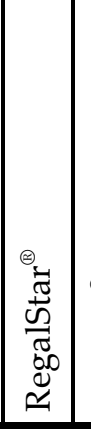 & 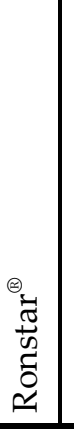 & $\stackrel{\Theta_{1}}{\stackrel{\vec{Z}}{0}}$ & 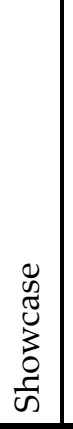 & 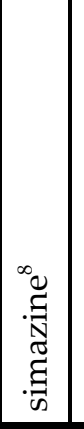 & 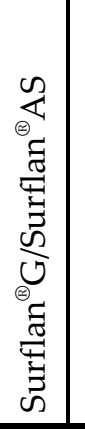 & 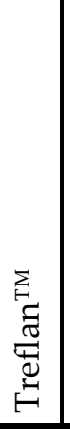 & 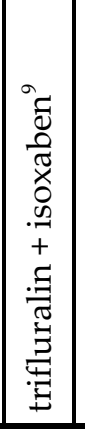 & 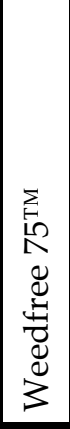 & $\stackrel{\oplus}{\times}$ \\
\hline Lepidium spp. ${ }^{10}$ & & & & $\mathrm{C}$ & $\mathrm{C}$ & & & $\mathrm{C}$ & & $\mathrm{C}$ & & $\mathrm{C}$ & $\mathrm{C}$ & C & $\mathrm{C}$ & & & & & & $S$ & & $\mathrm{C}$ & $\mathrm{C}$ & $\mathrm{C}$ & & $\mathrm{C}$ & $\mathrm{C}$ & C \\
\hline Marchantia polymorpha & & $\mathrm{S}$ & & $\mathrm{N}$ & $\mathrm{N}$ & & & $\mathrm{N}$ & & & & $\mathrm{S}$ & $\mathrm{N}$ & & $\mathrm{N}$ & & & $\mathrm{C}$ & $\mathrm{C}$ & & $S$ & & & $\mathrm{~N}$ & & & & & \\
\hline Medicago lupulina & & & & & & C & & C & C & & & & & & $S / C$ & & & & $\mathrm{~S}$ & & & & C & $\mathrm{S}$ & & & C & & \\
\hline Medicago trunculata & & & & & & & & & & & & & & & & & & & & & & C & & & & & & & \\
\hline Mollugo verticillata & $\mathrm{N}$ & $\mathrm{C}$ & $\mathrm{C}$ & $\mathrm{C}$ & $\mathrm{C}$ & $\mathrm{C}$ & $\mathrm{C}$ & $S$ & $\mathrm{C}$ & $\mathrm{C}$ & $\mathrm{C}$ & $\mathrm{C}$ & $\mathrm{C}$ & C & $\mathrm{C}$ & $\mathrm{C}$ & $\mathrm{C}$ & $\mathrm{C}$ & $\mathrm{C}$ & $\mathrm{C}$ & C & $\mathrm{C}$ & $\mathrm{C}$ & $\mathrm{C}$ & $\mathrm{C}$ & $\mathrm{S}$ & $S$ & $\mathrm{C}$ & C \\
\hline Murdannia nudiflora & & $\mathrm{C}$ & & & & & & & & & & & & & & & & & & & & & & & & & & & \\
\hline Oenothera spp..$^{10}$ & & & & $\mathrm{~N}$ & $\mathrm{C}$ & & & $\mathrm{S} / \mathrm{C}$ & C & & & $\mathrm{C}$ & $\mathrm{C}^{12}$ & & $\mathrm{C}$ & $\mathrm{S}$ & $\mathrm{S}$ & C & $\mathrm{N}$ & $\mathrm{N}$ & $\mathrm{S}$ & $\mathrm{C}$ & $\mathrm{C}$ & C & $\mathrm{C}$ & & $\mathrm{C}$ & & \\
\hline $\begin{array}{l}\text { Oxalis corniculata (synonym } \\
\text { O. stricta) }\end{array}$ & & C & & $\mathrm{N}$ & C & C & & C & C & & & C & C & C & C 1 & $|\mathrm{~N} / \mathrm{S}|$ & $\mathrm{N}$ & C & C & C & C & C & $\mathrm{C}$ & $\mathrm{N}$ & $\mathrm{C}$ & & C & C & C \\
\hline Phyllanthus tenellus & & $\mathrm{C}$ & & & & & & $\mathrm{N} / \mathrm{S}$ & & & & & & & S/C & $\mathrm{N}$ & & $\mathrm{S} / \mathrm{C}$ & $\mathrm{C} \mid \mathrm{s}$ & $\mathrm{S} / \mathrm{C}$ & $S$ & $\mathrm{~S}$ & $\mathrm{C}$ & & & & $\mathrm{S} / \mathrm{C}$ & & \\
\hline Phyllanthus urinaria & & & & & & & & $\mathrm{N} / \mathrm{S}$ & & & & & & $S$ & & $\mathrm{~N}$ & & $\mathrm{~S} / \mathrm{C}$ & C 5 & $\mathrm{~S} / \mathrm{C}$ & S & $\mathrm{S}$ & C & & & & $\mathrm{S} / \mathrm{C}$ & & \\
\hline Pilea microphylla & & & & & & & & & & & & & & & & & & & & & C & $\mathrm{C}$ & & & & & & & \\
\hline Pоа аппиа & $\mathrm{C}$ & $\mathrm{C}$ & & $\mathrm{C}$ & $\mathrm{C}$ & $\mathrm{C}$ & $\mathrm{C}$ & $\mathrm{N}$ & $S$ & $\mathrm{C}$ & & $\mathrm{C}$ & $S$ & C & $\mathrm{C}$ & C & C & $\mathrm{C}$ & $\mathrm{C}$ & & C & $\mathrm{C}$ & $\mathrm{C}$ & $\mathrm{C}$ & $\mathrm{C}$ & $\mathrm{C}$ & C & $\mathrm{C}$ & $\mathrm{C}$ \\
\hline Portulaca oleracea & $\mathrm{S}$ & $\mathrm{C}$ & $\mathrm{C}$ & $\mathrm{C}$ & $\mathrm{C}$ & $\mathrm{C}$ & $\mathrm{C}$ & $\mathrm{C}$ & & $\mathrm{C}$ & $\mathrm{C}$ & $\mathrm{C}$ & $\mathrm{C}$ & $\mathrm{C}$ & $\mathrm{C}$ & $\mathrm{S}$ & $\mathrm{C}$ & $\mathrm{C}$ & $\mathrm{C}$ & $\mathrm{C}$ & $\mathrm{C}$ & $\mathrm{C}$ & $\mathrm{C}$ & $\mathrm{C}$ & $\mathrm{C}$ & $\mathrm{C}$ & $\mathrm{C}$ & $\mathrm{C}$ & $\mathrm{C}$ \\
\hline Richardia scabra & & C & C & $\mathrm{N}$ & C & & C & $S$ & & & C & & & & $\mathrm{C}$ & C & $\mathrm{S}$ & & C & & & & $S$ & $\mathrm{C}$ & C & C & $S$ & C & $\mathrm{C}$ \\
\hline Senecio vulgaris & & $\mathrm{C}$ & & & & & & $\mathrm{C}$ & & & & $S$ & $S$ & $S$ & & & & & & & $S$ & $S$ & $C$ & $C$ & & & $\mathrm{C}$ & & \\
\hline Solanum spp. & $\mathrm{N}$ & $\mathrm{C}$ & $S$ & $\mathrm{~N}$ & $\mathrm{C}$ & & $\mathrm{C}$ & $\mathrm{C}$ & & $\mathrm{C}$ & $\mathrm{C}$ & & $\mathrm{C}$ & & $\mathrm{N}$ & $\mathrm{C}$ & & & & & & & $\mathrm{C}$ & $\mathrm{C}$ & $\mathrm{S}$ & $S$ & C & $\mathrm{C}$ & $\mathrm{S}$ \\
\hline Sonchus spp. ${ }^{10}$ & & $\mathrm{C}$ & $S$ & $\mathrm{C}$ & $\mathrm{C}$ & & & $\mathrm{C}$ & & & & $\mathrm{C}$ & $\mathrm{C}$ & $C$ & $\mathrm{C}$ & & $\mathrm{S}$ & $\mathrm{C}$ & $\mathrm{N}$ & $\mathrm{C}$ & $C$ & $\mathrm{C}$ & $\mathrm{C}$ & $\mathrm{S}$ & $\mathrm{S}$ & & C & $\mathrm{C}$ & $S$ \\
\hline Stachys floridana & & & & & & & & & & & & & & & & & & & $\mathrm{C}$ & & & & & $\mathrm{N}$ & & & & & \\
\hline Stellaria media & & $\mathrm{C}$ & $\mathrm{C}$ & $\mathrm{C}$ & $\mathrm{C}$ & $\mathrm{C}$ & $\mathrm{C}$ & $\mathrm{C}$ & $\mathrm{C}$ & $\mathrm{C}$ & & $\mathrm{C}$ & $S$ & $\mathrm{C}$ & $\mathrm{C} \mid \mathrm{I}$ & $\mathrm{N} / \mathrm{S}$ & $\mathrm{C}$ & $\mathrm{C}$ & $\mathrm{C}$ & $\mathrm{C}$ & $\mathrm{N}$ & $\mathrm{C}$ & $\mathrm{C}$ & $\mathrm{C}$ & $\mathrm{C}$ & $\mathrm{C}$ & $\mathrm{C}$ & $\mathrm{C}$ & $\mathrm{C}$ \\
\hline Vicia sativa & & & & & & & & & & & & & & & $S$ & & & & $\mathrm{~S}$ & & & & & $\mathrm{~S}$ & & & $\mathrm{C}$ & & \\
\hline
\end{tabular}




\begin{tabular}{|c|c|c|c|c|c|c|c|c|c|c|c|c|c|c|c|c|c|c|c|c|c|c|c|c|c|c|c|c|c|}
\hline Weed scientific name & 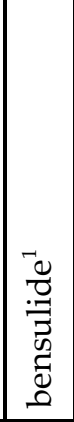 & 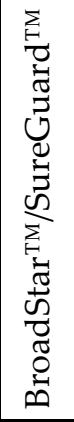 & 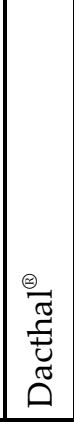 & 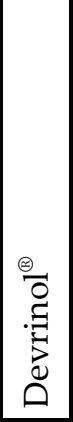 & 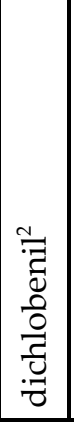 & 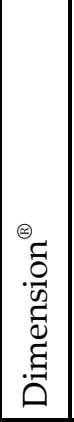 & 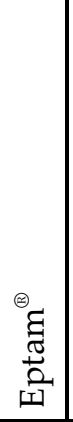 & 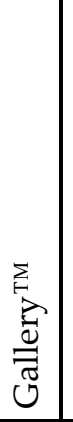 & $\left|\begin{array}{c}{ }^{\infty} \\
\infty \\
\Xi \\
\Xi \\
\Xi\end{array}\right|$ & 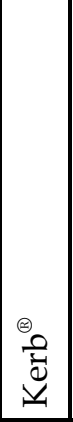 & 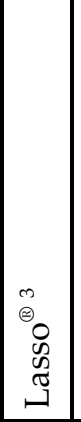 & $\stackrel{0}{T_{1}^{\prime}}$ & 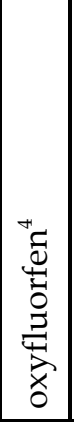 & 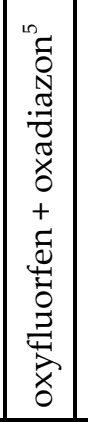 & 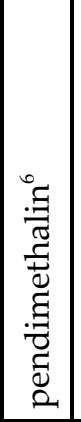 & 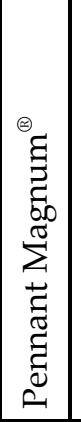 & 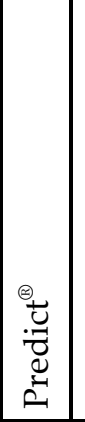 & 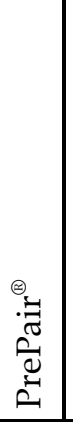 & 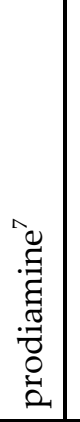 & 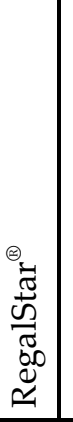 & 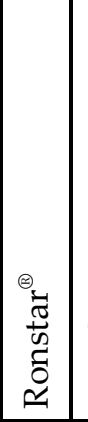 & \begin{tabular}{|}
$\stackrel{\oplus}{+}$ \\
$\vec{z}$ \\
$\stackrel{2}{\simeq}$
\end{tabular} & \begin{tabular}{|c|}
0 \\
0 \\
$\tilde{J}$ \\
3 \\
0 \\
$\tilde{E}$ \\
$\tilde{E}$
\end{tabular} & 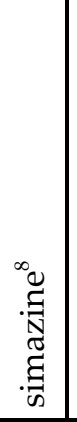 & 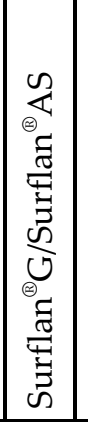 & 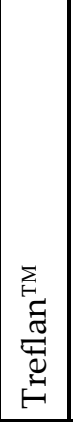 & 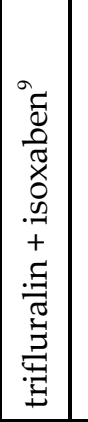 & 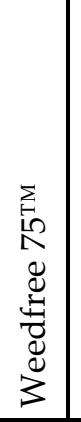 & \\
\hline \multicolumn{30}{|c|}{ 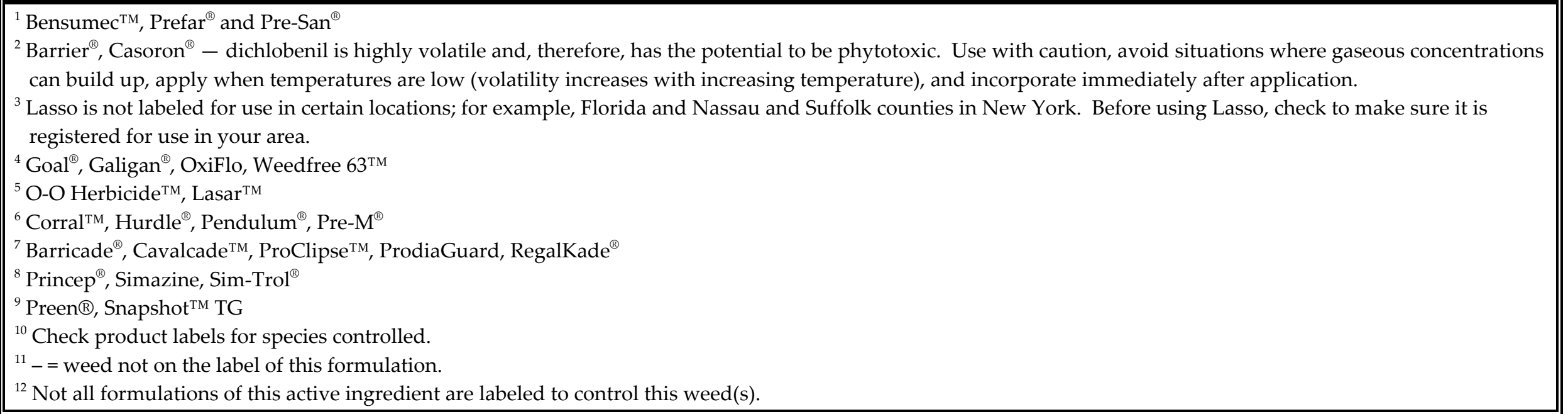 } \\
\hline
\end{tabular}




\begin{tabular}{|c|c|c|c|c|c|c|c|c|c|c|c|c|c|c|c|c|c|c|c|c|c|c|c|c|c|c|c|c|c|}
\hline Weed common name(s) & 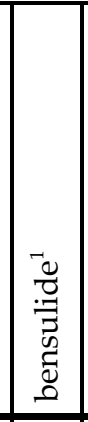 & 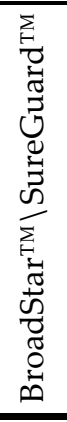 & 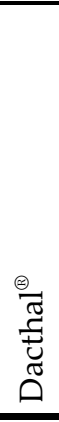 & 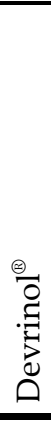 & 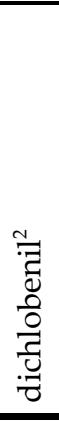 & 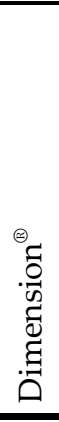 & 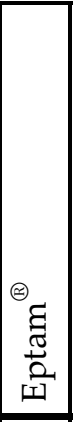 & 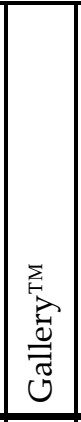 & $\begin{array}{l}\otimes_{0} \\
\infty \\
\Xi \\
\Xi \\
\end{array}$ & \begin{tabular}{|l}
$\Theta_{0}$ \\
$\stackrel{\vec{u}}{\Delta}$ \\
\end{tabular} & 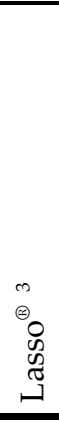 & \begin{tabular}{|l|}
$\stackrel{\ominus}{1}$ \\
$\stackrel{1}{0}$ \\
\end{tabular} & 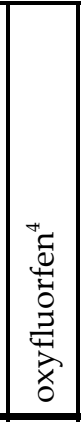 & 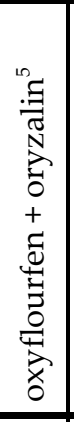 & 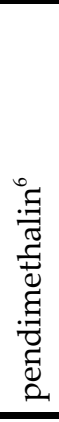 & 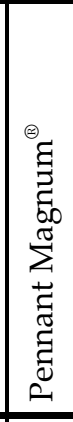 & 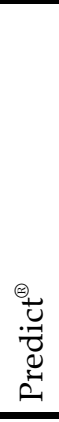 & 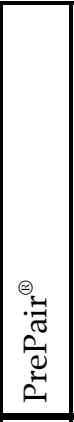 & 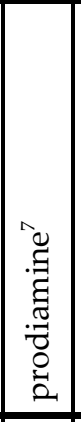 & 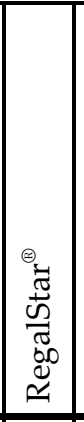 & 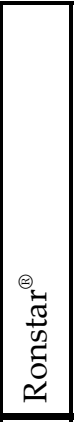 & $\begin{array}{l}\Theta_{1} \\
\overrightarrow{0} \\
\stackrel{0}{1} \\
\end{array}$ & 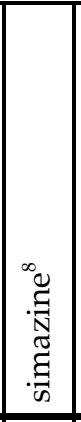 & \begin{tabular}{|c|} 
\\
0 \\
$\tilde{J}$ \\
3 \\
0 \\
$\tilde{E}$ \\
$\tilde{E}$ \\
\end{tabular} & 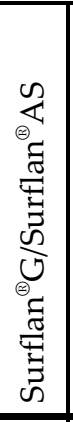 & 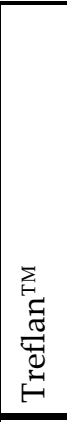 & 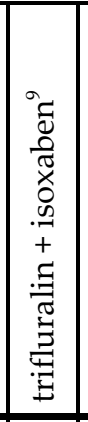 & 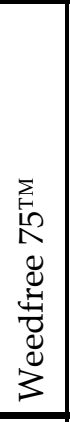 & $\stackrel{\ominus}{\times}$ \\
\hline artilleryweed & & & & & & & & & & & & & & & & & & & & & $\mathrm{C}$ & $\mathrm{C}$ & & & & & & & \\
\hline beggarweed, Florida & & $\mathrm{C}$ & & & & & & & & & $\mathrm{S}$ & & & & & & & & & & & & & & & & & & \\
\hline betony, Florida & & $\mathrm{C}$ & & & & & & & & & & & & & & & & & $\mathrm{S} / \mathrm{C}$ & & & & $\mathrm{N}$ & & & & & & \\
\hline bittercress $^{10}$ & & $\mathrm{C}$ & $\mathrm{N}$ & $\mathrm{C}$ & $\mathrm{C}$ & $\mathrm{C}$ & & $\mathrm{C}$ & $\mathrm{C}$ & & & $\mathrm{C}$ & $\mathrm{C}$ & $\mathrm{C}$ & $\mathrm{N}$ & $\mathrm{N}$ & & $\mathrm{C}$ & & $\mathrm{C}$ & $\mathrm{C}$ & $\mathrm{C}$ & $S$ & $\mathrm{C}$ & $\mathrm{C}$ & $\mathrm{N}$ & $\mathrm{C}$ & C & $\mathrm{C}$ \\
\hline bluegrass, annual & $\mathrm{C}$ & $\mathrm{C}$ & & $\mathrm{C}$ & $\mathrm{C}$ & $\mathrm{C}$ & $\mathrm{C}$ & $\mathrm{N}$ & $S$ & C & & $\mathrm{C}$ & $S$ & $\mathrm{C}$ & $\mathrm{C}$ & $\mathrm{C}$ & $\mathrm{C}$ & $\mathrm{C}$ & $\mathrm{C}$ & & C & $\mathrm{C}$ & $\mathrm{C}$ & C & C & C & C & $\mathrm{C}$ & $\mathrm{C}$ \\
\hline carpetweed & $\mathrm{N}$ & $\mathrm{C}$ & $\mathrm{C}$ & $\mathrm{C}$ & $\mathrm{C}$ & $\mathrm{C}$ & $\mathrm{C}$ & $S$ & $\mathrm{C}$ & C & $\mathrm{C}$ & $\mathrm{C}$ & $\mathrm{C}$ & C & $\mathrm{C}$ & $\mathrm{C}$ & $\mathrm{C}$ & $\mathrm{C}$ & $\mathrm{C}$ & $\mathrm{C}$ & $\mathrm{C}$ & $\mathrm{C}$ & $\mathrm{C}$ & C & $\mathrm{C}$ & $\mathrm{S}$ & $S$ & C & $\mathrm{C}$ \\
\hline carrot, wild & & & & $\mathrm{N}$ & $\mathrm{C}$ & & & $\mathrm{C}$ & & & & & & & & & & & & & & & $\mathrm{N}$ & $\mathrm{C}$ & $\mathrm{N}$ & & $\mathrm{C}$ & & $\mathrm{S} / \mathrm{C}$ \\
\hline chamberbitter & & & & & & & & $\mathrm{N} / \mathrm{S}$ & & & & & & $\mathrm{S}$ & & $\mathrm{N}$ & & $\mathrm{S} / \mathrm{C}$ & $\mathrm{C}$ & $\mathrm{S} / \mathrm{C}$ & $S$ & $\mathrm{~S}$ & & $\mathrm{C}$ & & & $\mathrm{S} / \mathrm{C}$ & & \\
\hline chamomile, stinking & & $\mathrm{C}$ & & & & & & $\mathrm{C}$ & & & & & $\mathrm{C}$ & & & & & & & & & & & $\mathrm{C}$ & & & $\mathrm{C}$ & & \\
\hline chickweed, common & & $\mathrm{C}$ & $\mathrm{C}$ & $\mathrm{C}$ & $\mathrm{C}$ & $\mathrm{C}$ & $\mathrm{C}$ & $\mathrm{C}$ & $\mathrm{C}$ & C & & $\mathrm{C}$ & $\mathrm{S}$ & $\mathrm{C}$ & $\mathrm{C}$ & $\mathrm{N} / \mathrm{S}$ & $\mathrm{C}$ & $\mathrm{C}$ & $\mathrm{C}$ & $\mathrm{C}$ & $\mathrm{N}$ & $\mathrm{C}$ & $\mathrm{C}$ & $\mathrm{C}$ & $\mathrm{C}$ & C & $\mathrm{C}$ & C & $\mathrm{C}$ \\
\hline chickweed, mouseear & & $\mathrm{C}$ & & $\mathrm{N}$ & $\mathrm{C}$ & & & $\mathrm{C}$ & $\mathrm{C}$ & C & & & & & $\mathrm{C}$ & & & & $\mathrm{C}$ & & & & $\mathrm{N}$ & $\mathrm{C}$ & $\mathrm{N}$ & & $\mathrm{C}$ & & \\
\hline crabgrass & $\mathrm{C}$ & $\mathrm{C}$ & $\mathrm{C}$ & $\mathrm{C}$ & $\mathrm{C}$ & $\mathrm{C}$ & C & $\mathrm{S}$ & $\mathrm{S}$ & C & $\mathrm{C}$ & $\mathrm{C}$ & $\mathrm{S} / \mathrm{C}$ & C & $\mathrm{C}$ & $\mathrm{C}$ & $\mathrm{C}$ & $\mathrm{C}$ & $\mathrm{C}$ & $\mathrm{C}$ & C & $\mathrm{C}$ & $\mathrm{C}$ & $\mathrm{C}$ & $\mathrm{C}$ & $\mathrm{C}$ & $\mathrm{C}$ & C & $\mathrm{C}$ \\
\hline crabweed, hairy & & & & & & & & & & & & $\mathrm{C}$ & & C & $S$ & $\mathrm{C}$ & & $\mathrm{C}$ & $S$ & & C & $\mathrm{C}$ & & & & & $\mathrm{N}$ & & $S$ \\
\hline cudweed & & & & & $\mathrm{C}$ & & & $\mathrm{C}$ & $\mathrm{S}$ & & & $\mathrm{C}$ & & C & $\mathrm{C}$ & & $\mathrm{C}$ & & & & & & $\mathrm{C}$ & $\mathrm{C}$ & & & C & & \\
\hline dayflower, spreading & & & & & & & & & & & & & & & & & $\mathrm{S}$ & & C & & & & & & & & & & \\
\hline dogfennel & & $\mathrm{C}$ & & & $\mathrm{C}$ & & & $\mathrm{C}$ & & & & $\mathrm{C}$ & & & $\mathrm{N}$ & & $\mathrm{C}$ & & $S^{3}$ & & $S$ & $\mathrm{C}$ & $\mathrm{C}$ & $\mathrm{C}$ & & & $\mathrm{C}$ & & \\
\hline doveweed & & $\mathrm{C}$ & & & & & & & & & & & & & & & & & & & & & & & & & & & \\
\hline eclipta & & $\mathrm{C}$ & & & & & & $\mathrm{C}$ & $\mathrm{C}$ & & & $\mathrm{C}$ & & & $\mathrm{N}$ & $\mathrm{S}$ & $\mathrm{C}$ & & & & $\mathrm{N}$ & $\mathrm{C}$ & & $\mathrm{C}$ & $\mathrm{C}$ & & $\mathrm{C}$ & & \\
\hline eveningprimrose & & & & $\mathrm{N}$ & $\mathrm{C}$ & & & $\mathrm{S} / \mathrm{C}$ & $\mathrm{C}$ & & & $\mathrm{C}$ & $C^{12}$ & & $\mathrm{C}$ & $S$ & $\mathrm{~S}$ & $\mathrm{C}$ & $\mathrm{N}$ & $\mathrm{N}$ & $S$ & $\mathrm{C}$ & $\mathrm{C}$ & $\mathrm{C}$ & $\mathrm{C}$ & & $\mathrm{C}$ & & \\
\hline geranium, Carolina & & $\mathrm{C}$ & & $\mathrm{C}$ & $\mathrm{C}$ & $\mathrm{C}$ & & $\mathrm{C}$ & $\mathrm{C}$ & & & & $C^{12}$ & & & & $\mathrm{C}$ & & & & & & $S$ & $\mathrm{C}$ & $\mathrm{C}$ & & $\mathrm{C}$ & & \\
\hline groundsel, common & & $\mathrm{C}$ & & & & & & $C$ & & & & $S$ & $S$ & $S$ & & & & & & & $S$ & $S$ & $\mathrm{C}$ & C & & & $\mathrm{C}$ & & \\
\hline goosegrass & $\mathrm{S} / \mathrm{C}$ & $\mathrm{C}$ & $\mathrm{S}$ & C & & $\mathrm{C}$ & C & $\mathrm{N}$ & & C & C & $\mathrm{C}$ & $\mathrm{S}$ & C & $\mathrm{C}$ & $\mathrm{S} / \mathrm{C}$ & $\mathrm{C}$ & C & C & $\mathrm{C}$ & $\mathrm{S} / \mathrm{C}$ & $\mathrm{C}$ & $\mathrm{S} / \mathrm{C}$ & C & $\mathrm{C}$ & C & C & C & C \\
\hline
\end{tabular}




\begin{tabular}{|c|c|c|c|c|c|c|c|c|c|c|c|c|c|c|c|c|c|c|c|c|c|c|c|c|c|c|c|c|c|}
\hline Weed common name(s) & 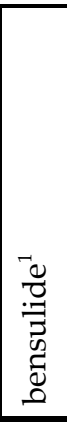 & 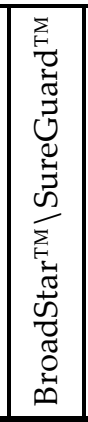 & 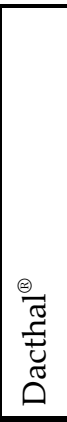 & 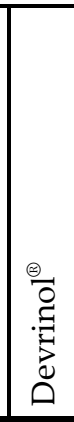 & 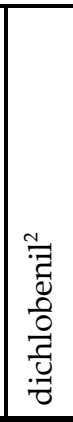 & 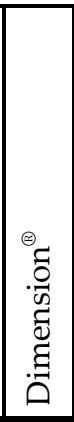 & $\stackrel{\circledast}{\circledR}$ & 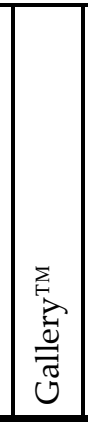 & \begin{tabular}{|l|}
$\Theta^{\otimes}$ \\
$\infty$ \\
$\Xi$ \\
$\Xi$ \\
$\Xi$
\end{tabular} & \begin{tabular}{|l}
$\otimes_{0}$ \\
$\stackrel{\vec{u}}{\vec{v}}$ \\
\end{tabular} & $\begin{array}{l}0 \\
0 \\
\Theta \\
0 \\
0 \\
0 \\
\Xi \\
\\
\end{array}$ & $\begin{array}{l}\stackrel{\oplus}{1} \\
\stackrel{1}{0} \\
0\end{array}$ & 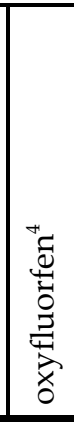 & 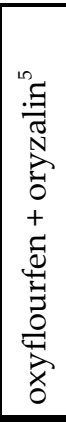 & 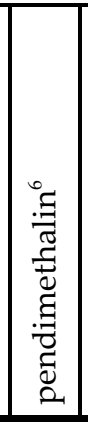 & 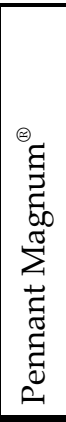 & 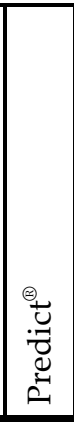 & 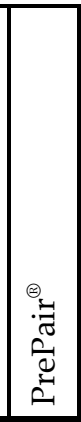 & 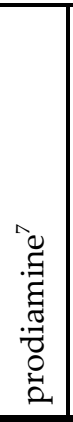 & 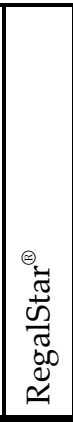 & 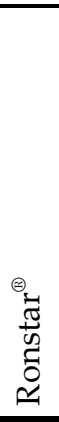 & 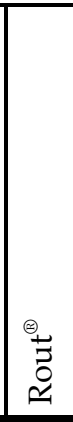 & 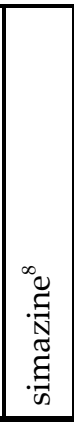 & 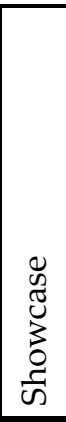 & 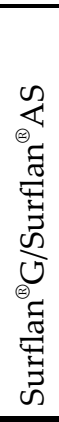 & 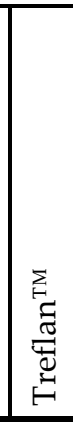 & 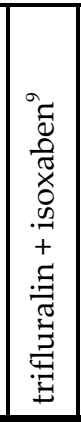 & 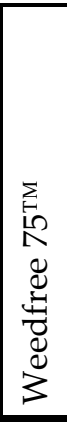 & $\begin{array}{l}{ }^{\ominus} \\
\bar{x}\end{array}$ \\
\hline kyllinga, green & $\mathrm{C}$ & C & C & & & C & $S$ & $\mathrm{C} / \mathrm{S}$ & & $\mathrm{S}$ & & & & & C & $\mathrm{C}$ & & & C & & $\mathrm{C}$ & & & & $\mathrm{C}$ & & & & $\mathrm{C}$ \\
\hline lambsquarters $^{10}$ & $\mathrm{C}$ & $\mathrm{C}$ & $\mathrm{C}$ & $\mathrm{C}$ & C & & $\mathrm{C}$ & $\mathrm{C}$ & & $\mathrm{C}$ & $\mathrm{S}$ & & $\mathrm{C}$ & & $\mathrm{C}$ & $S$ & $\mathrm{~S}$ & $\mathrm{C}$ & $\mathrm{C}$ & $\mathrm{C}$ & $\mathrm{C}$ & C & $\mathrm{C}$ & $\mathrm{C}$ & $\mathrm{C}$ & $\mathrm{C}$ & $\mathrm{C}$ & $\mathrm{C}$ & C \\
\hline liverwort, common & & $\mathrm{S}$ & & $\mathrm{N}$ & $\mathrm{N}$ & & & $\mathrm{N}$ & & & & $S$ & $\mathrm{~N}$ & & $\mathrm{~N}$ & & & $\mathrm{C}$ & C & & $\mathrm{S}$ & & $\mathrm{N}$ & & & & & & \\
\hline mayweed & & $\mathrm{C}$ & & & & & & $\mathrm{C}$ & & & & & $\mathrm{C}$ & & & & & & & & & & & $\mathrm{C}$ & & & $\mathrm{C}$ & $\mathrm{C}$ & \\
\hline medic. barrel & & & & & & & & & & & & & & & & & & & & & & $\mathrm{C}$ & & & & & & & \\
\hline medic, black & & & & & & $\mathrm{C}$ & & $\mathrm{C}$ & $\mathrm{C}$ & & & & & & $S / C$ & & & & $S$ & & & & $\mathrm{~S}$ & $\mathrm{C}$ & & & $\mathrm{C}$ & & \\
\hline $\operatorname{moss}^{10}$ & & $\mathrm{C}$ & & & & & & & & & & & & & & & & & & & & $\mathrm{C}$ & & & & & & & \\
\hline mulberry weed & & $\mathrm{C}$ & & & & & & & & & & $\mathrm{C}$ & & $\mathrm{C}$ & $S$ & $\mathrm{C}$ & & $\mathrm{C}$ & $\mathrm{S}$ & & $\mathrm{C}$ & $\mathrm{C}$ & & & & & $\mathrm{N}$ & & $S$ \\
\hline mustard $^{10}$ & & & $\mathrm{~N}$ & $S$ & $\mathrm{C}$ & $\mathrm{C}$ & & $\mathrm{C}$ & & $C$ & $\mathrm{~N}$ & & $\mathrm{C}$ & & & $\mathrm{N}$ & & & & & $\mathrm{C}$ & C & $\mathrm{C}$ & $C$ & $S$ & & $C$ & $C$ & $S$ \\
\hline nightshade, black & $\mathrm{N}$ & $\mathrm{C}$ & $S$ & $\mathrm{~N}$ & $\mathrm{C}$ & & $\mathrm{C}$ & $\mathrm{C}$ & & C & $\mathrm{C}$ & & C & & $\mathrm{N}$ & $\mathrm{C}$ & & & & & & & $\mathrm{C}$ & $\mathrm{C}$ & $\mathrm{S}$ & $\mathrm{S}$ & $\mathrm{C}$ & $C$ & $S$ \\
\hline nutsedge, purple & & & $\mathrm{N}$ & & & & $\mathrm{C}$ & & $\mathrm{S} / \mathrm{C}$ & & & & & & & & $S$ & & & & & & & & & & & & \\
\hline nutsedge, yellow & $\mathrm{N}$ & & $\mathrm{N}$ & $S$ & $\mathrm{C}$ & & $\mathrm{C}$ & $\mathrm{N}$ & $\mathrm{S} / \mathrm{C}$ & $\mathrm{N}$ & $S$ & $\mathrm{~N}$ & $\mathrm{~N}$ & & $\mathrm{~N}$ & $\mathrm{C}$ & $\mathrm{S}$ & & $\mathrm{N}$ & $\mathrm{N}$ & $\mathrm{N}$ & $\mathrm{N}$ & & $\mathrm{N}$ & $\mathrm{N}$ & $\mathrm{N}$ & & & \\
\hline pepperweed $^{10}$ & & & & $\mathrm{C}$ & $\mathrm{C}$ & & & C & & C & & C & $\mathrm{C}$ & $\mathrm{C}$ & $\mathrm{C}$ & & & & & & $S$ & & $\mathrm{C}$ & $\mathrm{C}$ & $\mathrm{C}$ & & $\mathrm{C}$ & $\mathrm{C}$ & $\mathrm{C}$ \\
\hline phyllanthus, long-stalked & & $\mathrm{C}$ & & & & & & $\mathrm{N} / \mathrm{S}$ & & & & & & & $\mathrm{S} / \mathrm{C}$ & $\mathrm{N}$ & & $\mathrm{S} / \mathrm{C}$ & $\mathrm{C}$ & $\mathrm{S} / \mathrm{C}$ & $S$ & $\mathrm{~S}$ & & $\mathrm{C}$ & & & $\mathrm{S} / \mathrm{C}$ & & \\
\hline pigweeds $^{10}$ & $\mathrm{C}$ & $\mathrm{C}$ & $\mathrm{S}$ & $\mathrm{C}$ & $\mathrm{C}$ & $\mathrm{C}$ & $\mathrm{C}$ & $\mathrm{C}$ & $\mathrm{C}$ & & $\mathrm{C}$ & $\mathrm{C}$ & $\mathrm{C}$ & $\mathrm{C}$ & $\mathrm{C}$ & $\mathrm{C}$ & $\mathrm{C}$ & $\mathrm{C}$ & $\mathrm{C}$ & $\mathrm{C}$ & $\mathrm{C}$ & $\mathrm{C}$ & $\mathrm{C}$ & $\mathrm{C}$ & $\mathrm{C}$ & $\mathrm{C}$ & $\mathrm{C}$ & C & $\mathrm{C}$ \\
\hline poinsettia, wild & & $\left.\right|^{11} / \mathrm{C}$ & & & & & & & & & & $\mathrm{C}$ & & & & & & & & & & & & & & & & & \\
\hline purslane, common & $\mathrm{S}$ & $\mathrm{C}$ & $\mathrm{C}$ & $\mathrm{C}$ & $\mathrm{C}$ & $\mathrm{C}$ & $\mathrm{C}$ & $\mathrm{C}$ & & $\mathrm{C}$ & $\mathrm{C}$ & $\mathrm{C}$ & $\mathrm{C}$ & $\mathrm{C}$ & $\mathrm{C}$ & $\mathrm{S}$ & $\mathrm{C}$ & $\mathrm{C}$ & $\mathrm{C}$ & $\mathrm{C}$ & $\mathrm{C}$ & $\mathrm{C}$ & $\mathrm{C}$ & $\mathrm{C}$ & $\mathrm{C}$ & $\mathrm{C}$ & $\mathrm{C}$ & C & $\mathrm{C}$ \\
\hline pusley, Florida & & $\mathrm{C}$ & C & $\mathrm{N}$ & $\mathrm{C}$ & & $\mathrm{C}$ & $\mathrm{S}$ & & & $\mathrm{C}$ & & & & $\mathrm{C}$ & $\mathrm{C}$ & $S$ & & $\mathrm{C}$ & & & & $\mathrm{C}$ & $\mathrm{S}$ & $\mathrm{C}$ & $\mathrm{C}$ & $\mathrm{S}$ & C & $\mathrm{C}$ \\
\hline ragweed, common & $\mathrm{N}$ & $S$ & $\mathrm{~N}$ & $\mathrm{~S} / \mathrm{C}$ & $\mathrm{C}$ & & & $\mathrm{C}$ & & $\mathrm{C}$ & $S$ & & $S$ & & $\mathrm{~N}$ & $\mathrm{~S}$ & $\mathrm{~S}$ & $\mathrm{C}$ & $\mathrm{S}$ & & $\mathrm{C}$ & & $\mathrm{C}$ & $\mathrm{C}$ & $S$ & $\mathrm{~N}$ & $\mathrm{C}$ & & $\mathrm{S}$ \\
\hline sedge, annual & & & & & & & & $\mathrm{N}$ & $\mathrm{S}$ & & & & & & & & $\mathrm{C}$ & $\mathrm{C}$ & & & $\mathrm{C}$ & & & & & & & & \\
\hline sedge, globe & & & & & & & & & $\mathrm{C}$ & & & & & & & & & & & & & & & & & & & & \\
\hline shepherdspurse & C & $\mathrm{C}$ & $\mathrm{S}$ & $\mathrm{C}$ & $\mathrm{C}$ & $\mathrm{C}$ & & $\mathrm{C}$ & & $\mathrm{C}$ & & $\mathrm{C}$ & $\mathrm{S} / \mathrm{C}$ & $\mathrm{C}$ & $\mathrm{C}$ & & $\mathrm{C}$ & $\mathrm{C}$ & $\mathrm{C}$ & & $\mathrm{C}$ & $\mathrm{C}$ & $\mathrm{C}$ & $\mathrm{C}$ & $\mathrm{C}$ & & $\mathrm{C}$ & C & $\mathrm{C}$ \\
\hline Spanish needles & & & & & $\mathrm{C}$ & & & & & & & & & & & & & & $\mathrm{N}$ & & & & $\mathrm{C}$ & & & & $\mathrm{S} / \mathrm{C}$ & & \\
\hline
\end{tabular}




\begin{tabular}{|c|c|c|c|c|c|c|c|c|c|c|c|c|c|c|c|c|c|c|c|c|c|c|c|c|c|c|c|c|c|}
\hline Weed common name(s) & 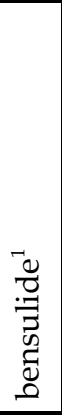 & 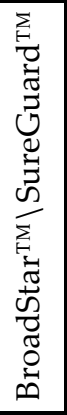 & 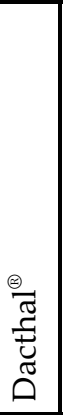 & 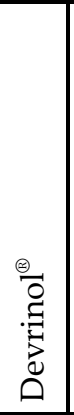 & $\begin{array}{l}7 \\
\overline{0} \\
0 \\
0 \\
0 \\
\overline{0} \\
: 0 \\
\overline{0}\end{array}$ & 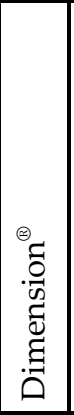 & 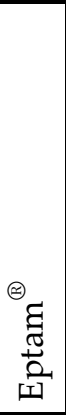 & 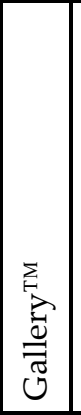 & $\left|\begin{array}{l}\Theta_{\infty} \\
\infty \\
\tilde{\Xi} \\
\Xi\end{array}\right|$ & 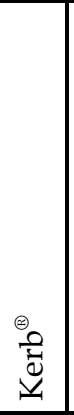 & 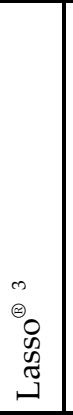 & $\begin{array}{l}\stackrel{\oplus}{1} \\
\mathbb{1} \\
0\end{array}$ & 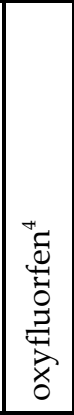 & 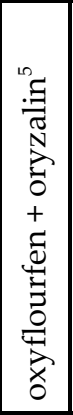 & 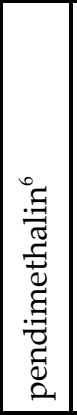 & 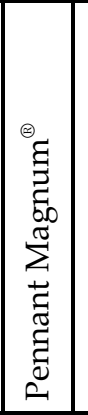 & 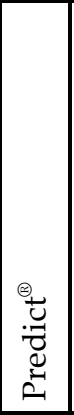 & 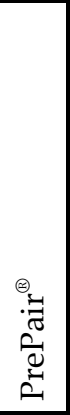 & 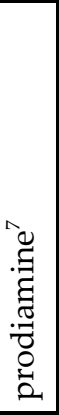 & 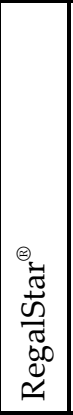 & $\begin{array}{c}\oplus_{\overrightarrow{1}} \\
\tilde{\tilde{w}} \\
\tilde{\omega} \\
\tilde{0} \\
\simeq\end{array}$ & $\begin{array}{l}\theta_{1} \\
\overrightarrow{0} \\
\cong\end{array}$ & 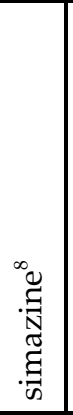 & 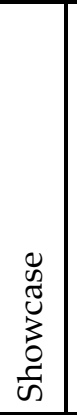 & 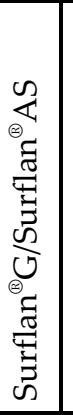 & 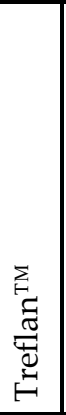 & 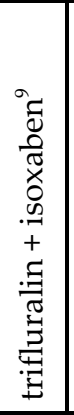 & 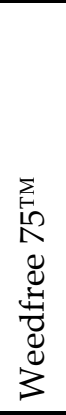 & $\stackrel{\ominus}{\underset{x}{*}}$ \\
\hline sowthistle & & & $\mathrm{S}$ & C & C & & & C & & & & C & C & C & C & & $\mathrm{S}$ & C & $\mathrm{N}$ & C & C & C & $\mathrm{S}$ & C & S & & C & C & $\mathrm{S}$ \\
\hline spurge, garden & $\mathrm{N}$ & $\mathrm{C}$ & & & & $\mathrm{C}$ & & & & $\mathrm{N}$ & & $\mathrm{C}$ & $C^{12}$ & $\mathrm{C}$ & & & & $\mathrm{C}$ & & & & $\mathrm{C}$ & & & & & & & \\
\hline spurge, hairy & & & & & & & & & & & & & & & & & & & & & & $\mathrm{C}$ & & & & & & & \\
\hline spurge, prostrate & & $C$ & $S$ & $\mathrm{~N}$ & $\mathrm{C}$ & $\mathrm{C}$ & & $\mathrm{C}$ & & & & $\mathrm{C}$ & $\mathrm{C}$ & $\mathrm{C}$ & $\mathrm{C}$ & $S$ & $\mathrm{C}$ & $\mathrm{C}$ & $C$ & $S$ & $S$ & $\mathrm{C}$ & $S$ & $\mathrm{C}$ & $\mathrm{C}$ & & $\mathrm{C}$ & $\mathrm{C}$ & $\mathrm{S} / \mathrm{C}$ \\
\hline spurge, spotted & & $C$ & $\mathrm{~S}$ & $\mathrm{~N}$ & $\mathrm{C}$ & $\mathrm{C}$ & & $\mathrm{S} / \mathrm{C}$ & & & & $\mathrm{C}$ & $\mathrm{C}$ & $\mathrm{C}$ & $\mathrm{C}$ & & $S$ & & $C$ & $\mathrm{C}$ & $\mathrm{S}$ & $\mathrm{C}$ & $\mathrm{S}$ & $\mathrm{C}$ & $S$ & & $\mathrm{C}$ & & $S$ \\
\hline ticktrefoil, Dixie & & C & & & & & & & & & $S$ & & & & & & & & & & & & & & & & & & \\
\hline vetch, common & & & & & & & & & & & & & & & $S$ & & & & $S$ & & & & $S$ & & & & $\mathrm{C}$ & & \\
\hline woodsorrel, yellow & & C & & $\mathrm{N}$ & C & $\mathrm{C}$ & & C & C & & & C & $\mathrm{C}$ & $\mathrm{C}$ & $\mathrm{C}$ & $\mathrm{N} / \mathrm{S}$ & $\mathrm{N}$ & $\mathrm{C}$ & $\mathrm{C}$ & $\mathrm{C}$ & C & C & $\mathrm{N}$ & C & C & & $\mathrm{C}$ & C & $C$ \\
\hline \multicolumn{30}{|c|}{ 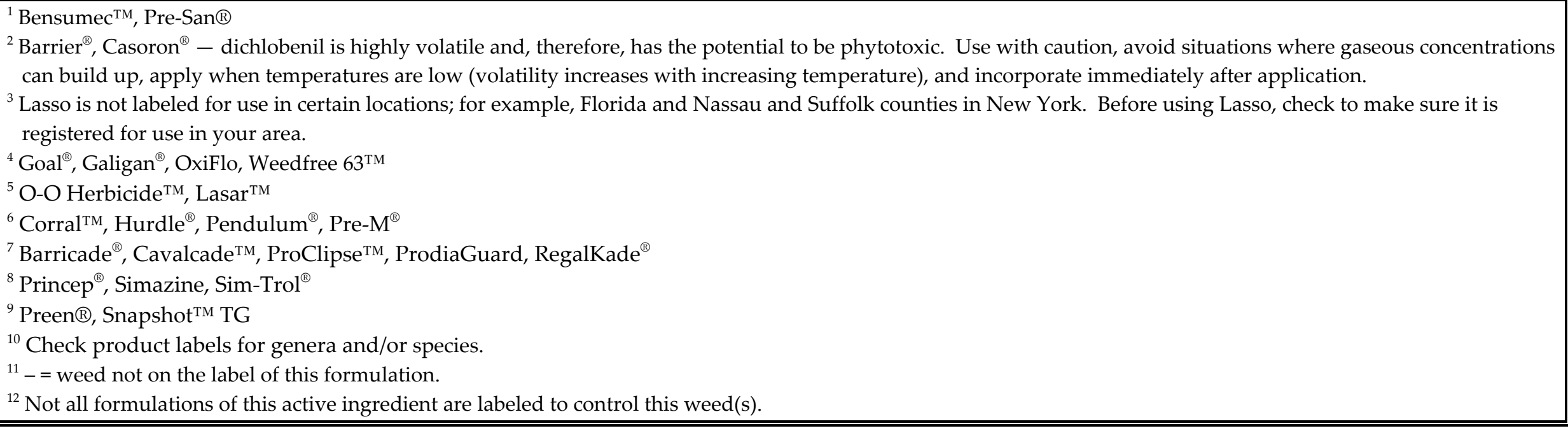 } \\
\hline
\end{tabular}




\begin{tabular}{|c|c|c|c|c|c|c|c|c|c|c|c|c|c|c|c|c|c|c|c|c|c|c|c|c|c|c|c|c|c|}
\hline Scientific name & 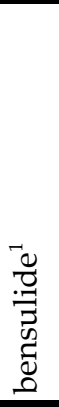 & 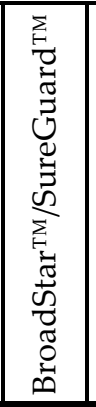 & 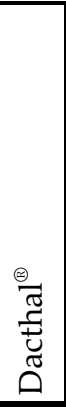 & 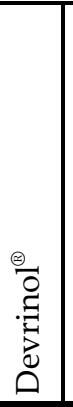 & 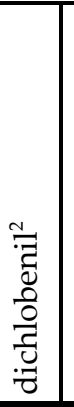 & 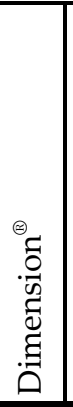 & 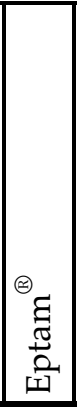 & 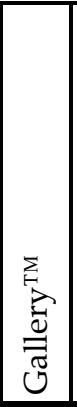 & $\begin{array}{l}\Theta_{0} \\
\infty \\
\Xi \\
\Xi\end{array}$ & $\begin{array}{l}\oplus_{0} \\
\stackrel{0}{\vec{u}} \\
\underline{\underline{u}}\end{array}$ & 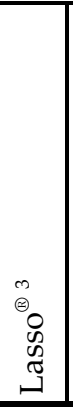 & $\stackrel{\circledast}{\stackrel{\oplus}{1}}$ & 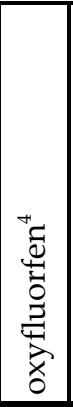 & 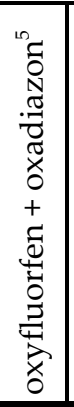 & 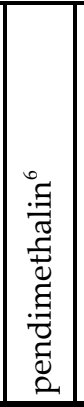 & 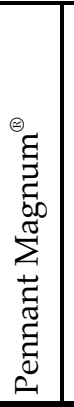 & 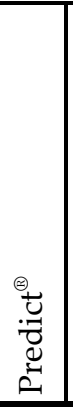 & 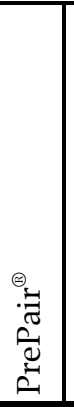 & 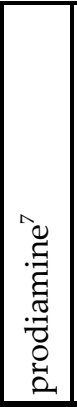 & 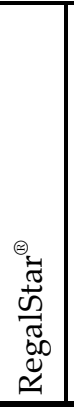 & 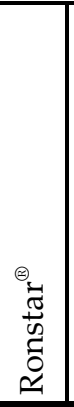 & 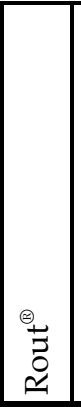 & 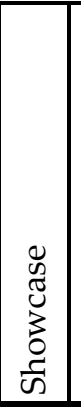 & 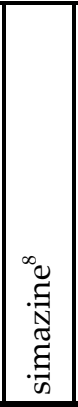 & 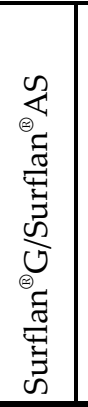 & 离 & 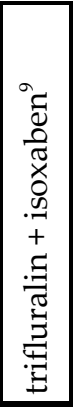 & 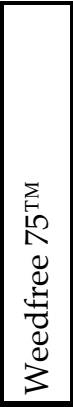 & $\stackrel{\ominus}{\stackrel{\oplus}{\times}}$ \\
\hline \multicolumn{30}{|l|}{ TREES AND SHRUBS } \\
\hline Abelia $\times$ grandiflora & $\mathrm{Y}$ & $\mathrm{Y} / \mathrm{-}^{10}$ & $\mathrm{Y}$ & $\mathrm{Y}$ & & $\mathrm{Y}$ & & $\mathrm{Y}$ & $\mathrm{N}$ & & & $\mathrm{Y}$ & & $\mathrm{Y}$ & $\mathrm{Y}$ & $\mathrm{Y}$ & & & $\bar{Y}$ & & & $\bar{Y}$ & $\bar{Y}$ & & $\mathrm{Y} / \mathrm{Y}$ & $\mathrm{Y}$ & $\mathrm{Y}$ & & $\mathrm{Y}$ \\
\hline Acacia spp. & & $\mathrm{Y} /-$ & & & & & & $\mathrm{Y}^{11}$ & & & & $\mathrm{Y}$ & & $\mathrm{Y}$ & $\mathrm{Y}^{11}$ & & & & & $\mathrm{Y}$ & $\mathrm{Y}$ & & $\mathrm{Y}$ & & & $Y^{11}$ & $\mathrm{Y}^{11}$ & & $\mathrm{Y}^{11}$ \\
\hline Acalypha wilkesiana & & & & & & & & $\mathrm{Y}$ & & & & & & & & & & & & & & & & & & $\bar{Y}$ & $\mathrm{Y}$ & & \\
\hline Acca sellowiana & & & & & & $\mathrm{Y}$ & & & & & & & & & & & & & & & & & & & & $\bar{Y}$ & & & \\
\hline Acer ginnala & & $\mathrm{Y} /-$ & & & & & & $\bar{Y}$ & & & & & & & & & & & & & & & $\bar{Y}$ & & $\mathrm{Y} / \mathrm{Y}$ & $\mathrm{Y}$ & $\mathrm{Y}$ & & \\
\hline Acer palmatum & & \begin{tabular}{|l|}
$\mathrm{Y} /-$ \\
\end{tabular} & & & & $\mathrm{Y}$ & & & & & & & & & $\bar{Y}$ & & & & $\bar{Y}$ & $\mathrm{Y}$ & $\mathrm{Y}$ & & $\bar{Y}$ & & & & & & \\
\hline Acer pensylvanicum & & $\mathrm{Y} /-$ & & & & & & & & & & & & & & & & & & & & & & & & & & & \\
\hline Acer rubrum & & $\mathrm{Y} /-$ & & & & $\mathrm{Y}$ & & $\mathrm{Y}$ & & & & & $\mathrm{Y}$ & & $\mathrm{Y}$ & & $\mathrm{Y}$ & & & & $\mathrm{Y}$ & & $\mathrm{Y}$ & & $\mathrm{Y} / \mathrm{Y}$ & $\mathrm{Y}$ & $\mathrm{Y}$ & & \\
\hline Acer saccharinum & & & & & & $\mathrm{Y}$ & & $\mathrm{Y}$ & & & & & & & & & & & & & $\mathrm{Y}$ & & $\mathrm{Y}$ & & $\mathrm{Y} / \mathrm{Y}$ & $\mathrm{Y}$ & $\mathrm{Y}$ & & \\
\hline Acer saccharum & & & & & & $\mathrm{Y}$ & & & & & & & $\mathrm{Y}$ & & $\bar{Y}$ & & & & & & & & & & & $\bar{Y}$ & & & \\
\hline Acer spp. & & $-/ Y$ & $\mathrm{Y}$ & $\mathrm{Y}$ & $\mathrm{Y}$ & $\mathrm{Y}^{11}$ & & & & $\mathrm{Y}$ & & $\mathrm{Y}$ & $\mathrm{Y}^{11}$ & $\mathrm{Y}$ & $\mathrm{Y}^{11}$ & $\mathrm{Y}$ & & $\mathrm{Y}$ & $\mathrm{Y}^{11}$ & & $\mathrm{Y}$ & & & $\mathrm{Y}$ & $\mathrm{Y} / \mathrm{Y}$ & $\mathrm{Y}^{11}$ & & & $\mathrm{Y}$ \\
\hline Aesculus pavia & & & $\mathrm{Y}$ & & & & & & & & & & & & $\mathrm{Y}$ & & & & & & & & & & & & & & \\
\hline \multicolumn{30}{|l|}{$\begin{array}{l}\text { Afrocarpus gracilior } \\
\text { (synonym Podocarpus } \\
\text { gracilior) }\end{array}$} \\
\hline Allamanda cathartica & & & & & & & & & & & & & & & & & & & & & $\mathrm{Y}$ & & & & & & & & \\
\hline Araucaria heterophylla & & & & & & & & $\mathrm{Y}$ & & & & $\mathrm{Y}$ & & $\mathrm{Y}$ & & & & & & & & & & & & & & & \\
\hline Astilbe spp. & & & & & & & & & & & & & & & & & & & & & & & & & $\mathrm{Y} / \mathrm{Y}$ & $Y^{11}$ & $\mathrm{Y}^{11}$ & & $Y^{11}$ \\
\hline Aucuba japonica & & & $\mathrm{Y}$ & & & & & & & & & & & & $\mathrm{Y}$ & $\mathrm{Y}$ & & & $\mathrm{Y}$ & & & & & $\mathrm{Y}$ & & & & & \\
\hline Bauhinia galpinii & & & & & & & & $\mathrm{Y}$ & & & & & & & & & & & & & & & & & & & & & \\
\hline Berberis julianae & & & & & & & & & & & & & & & & $\mathrm{Y}$ & & & $\mathrm{Y}$ & & & & & & & & & & \\
\hline Berberis thunbergii & & & $\mathrm{Y}$ & & & $\mathrm{Y}$ & & $\mathrm{Y}$ & & & & & & & $\mathrm{Y}$ & $\mathrm{Y}$ & & & $\mathrm{Y}$ & & & $\mathrm{Y}^{11}$ & & & $\mathrm{Y} / \mathrm{Y}$ & $\mathrm{Y}$ & $\mathrm{Y}$ & & $Y^{11}$ \\
\hline Berberis $\times$ gladwynensis & & & & & & & & $\mathrm{Y}$ & & & & & & & & & & & $\mathrm{Y}$ & $\mathrm{Y}$ & & & & & & $\mathrm{Y}$ & $\mathrm{Y}$ & & \\
\hline
\end{tabular}




\begin{tabular}{|c|c|c|c|c|c|c|c|c|c|c|c|c|c|c|c|c|c|c|c|c|c|c|c|c|c|c|c|c|c|}
\hline Scientific name & 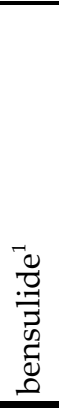 & 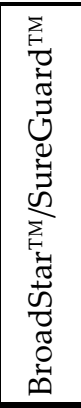 & 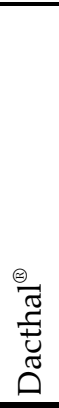 & 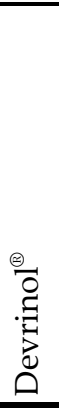 & 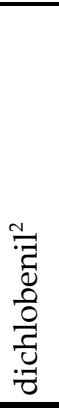 & 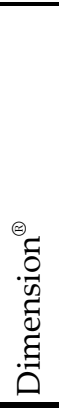 & 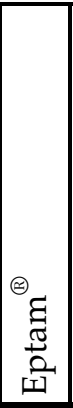 & 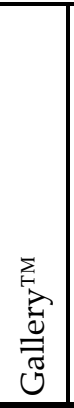 & $\begin{array}{l}\otimes_{0} \\
\infty \\
\Xi \\
\Xi\end{array}$ & 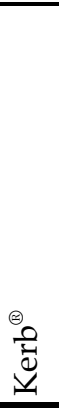 & 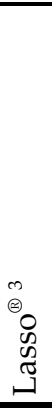 & ֻै & 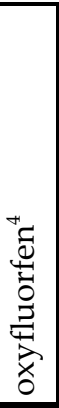 & 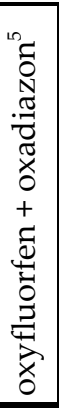 & 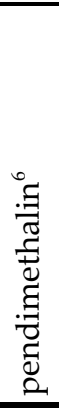 & 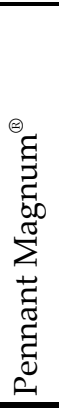 & 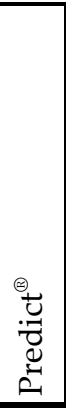 & 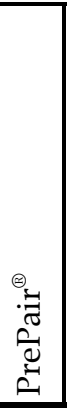 & 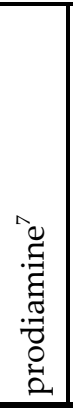 & 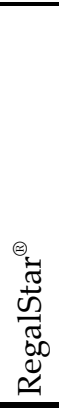 & 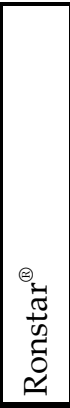 & 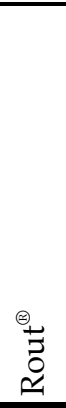 & $\begin{array}{l}\tilde{y} \\
\text { J } \\
\vdots \\
3 \\
0 \\
\text { ऊ }\end{array}$ & 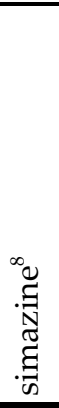 & 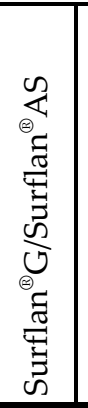 & 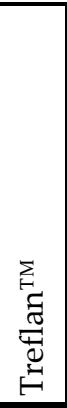 & 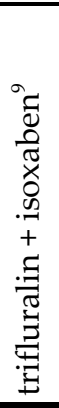 & 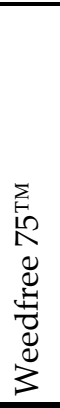 & $\begin{array}{c} \\
\stackrel{\oplus}{\times} \\
\stackrel{\times}{\times} \\
\end{array}$ \\
\hline Berberis spp. & & $\mathrm{Y} /-$ & & & $\mathrm{Y}$ & $Y^{11}$ & $\mathrm{Y}$ & $\mathrm{Y}^{11}$ & & $\mathrm{Y}$ & & $\mathrm{Y}$ & & $\mathrm{Y}$ & $Y^{11}$ & $\mathrm{Y}$ & & & $\mathrm{Y}^{11}$ & & $\mathrm{Y}$ & & $\mathrm{Y}$ & $\mathrm{Y}$ & & $\mathrm{Y}^{11}$ & $Y^{11}$ & & \\
\hline Betula nigra & & & $\mathrm{Y}$ & $\mathrm{Y}$ & $\mathrm{Y}$ & $\mathrm{Y}$ & & $\mathrm{Y}$ & & $\mathrm{Y}$ & & $\mathrm{Y}$ & $\mathrm{Y}$ & $\mathrm{Y}$ & $\mathrm{Y}$ & $\mathrm{Y}$ & & & & & & & $\mathrm{Y}$ & & $\mathrm{Y} / \mathrm{Y}$ & $\mathrm{Y}$ & $\mathrm{Y}$ & & $\mathrm{Y}$ \\
\hline Betula spp. & & $\mathrm{Y} / \mathrm{Y}$ & & & & & & & & & & & & & & & & & & & & & & & & & & & \\
\hline Bougainvillea spp. & & & & $\mathrm{Y}$ & & & & $\mathrm{Y}^{11}$ & & & & & & & & $\mathrm{Y}$ & & $\mathrm{Y}$ & $\mathrm{Y}$ & & $Y^{11}$ & $\mathrm{Y}$ & $\mathrm{Y}$ & & $Y / Y^{11}$ & $\mathrm{Y}^{11}$ & $\mathrm{Y}^{11}$ & & $\mathrm{Y}$ \\
\hline Buddleja davidii & & & & & & & & & & & & & & & & & & & Y & & & & & & & & & & \\
\hline Buddleja spp. & & $\mathrm{Y} /-$ & & & & & & & & & & & & & & & & & & & & & & & & & & & \\
\hline Buxus microphylla & & & & & & & & $\mathrm{Y}$ & & & & & & & $\mathrm{Y}$ & & & $\mathrm{Y}^{11}$ & $\mathrm{Y}$ & $\mathrm{Y}$ & $\mathrm{Y}$ & $\mathrm{Y}$ & $\mathrm{Y}$ & & $\mathrm{Y} / \mathrm{Y}$ & $\mathrm{Y}$ & $\mathrm{Y}$ & & $\mathrm{Y}$ \\
\hline Buxus spp. & $\mathrm{Y}$ & $\mathrm{Y} /-$ & $\mathrm{Y}$ & $\mathrm{Y}$ & $\mathrm{Y}$ & $\mathrm{Y}^{11}$ & & $\mathrm{Y}^{11}$ & & $\mathrm{Y}$ & & $\mathrm{Y}$ & & $\mathrm{Y}$ & & $\mathrm{Y}$ & $\mathrm{Y}^{11}$ & $\mathrm{Y}^{11}$ & & & $\mathrm{Y}^{11}$ & $Y^{11}$ & $\mathrm{Y}$ & & $Y / Y^{11}$ & $\mathrm{Y}^{11}$ & $\mathrm{Y}^{11}$ & & $\mathrm{Y}^{11}$ \\
\hline Callistemon citrinus & & & & $\mathrm{Y}$ & & $\mathrm{Y}$ & & $\mathrm{Y}$ & & & & $\mathrm{Y}$ & & $\mathrm{Y}$ & $\mathrm{Y}$ & & & & $\mathrm{Y}$ & & $\mathrm{Y}^{11}$ & & $\mathrm{Y}$ & & $\mathrm{Y} / \mathrm{Y}$ & $\mathrm{Y}$ & $\mathrm{Y}$ & & $\mathrm{Y}$ \\
\hline Callistemon viminalis & & & & $\mathrm{Y}$ & & & & $\mathrm{Y}$ & & & & $\mathrm{Y}$ & & $\mathrm{Y}$ & & & & & $\mathrm{Y}$ & $\mathrm{Y}$ & & & $\mathrm{Y}$ & & & $\mathrm{Y}$ & $\mathrm{Y}$ & & \\
\hline Callistemon spp. & & $\mathrm{Y} /-$ & & & & & & & & & & & & & & & & & & & & & & & & & & & \\
\hline Camellia japonica & & & & & & $Y^{11}$ & & $\mathrm{Y}$ & & & & & & & $\mathrm{Y}$ & & & $\mathrm{Y}$ & & $\mathrm{Y}$ & $\mathrm{Y}$ & & $\mathrm{Y}$ & & & $Y$ & $\mathrm{Y}$ & & \\
\hline Camellia sasanqua & & & & & & $\mathrm{Y}$ & & & & & & & & & & & & $\mathrm{Y}$ & & & $\mathrm{Y}$ & & & & & $\mathrm{Y}$ & & & \\
\hline Camellia spp. & & $\mathrm{Y} /-$ & $\mathrm{Y}$ & $\mathrm{Y}$ & $\mathrm{Y}$ & & & & & & & $\mathrm{Y}$ & & $\mathrm{Y}$ & & $\mathrm{Y}$ & & $\mathrm{Y}$ & & & & & & & & & & & \\
\hline $\begin{array}{l}\text { Carissa macrocarpa } \\
\text { (synonym C. grandiflora) }\end{array}$ & & & & $\mathrm{Y}$ & & & & & & & & & & & Y & & & $\mathrm{Y}$ & & & $\mathrm{Y}$ & $\mathrm{Y}$ & & & & & & & \\
\hline Carya spp. & & $Y^{11 / Y}$ & & & & & & & & & & & $\mathrm{Y}$ & & & & & & & & & & & & $-/ Y$ & & & & \\
\hline Caryota spp. & & & & & & & & & & & & & & & & & & $\mathrm{Y}$ & & & & & & & & & & & \\
\hline Castanea spp. & & $\mathrm{Y} / \mathrm{Y}$ & $\mathrm{Y}$ & & & & & & & & & & $\mathrm{Y}$ & & & & & & & & & & & & & $\mathrm{Y}^{11}$ & & & \\
\hline Cercis canadensis & & $\mathrm{Y} / \mathrm{Y}$ & $\mathrm{Y}$ & & & & & $\mathrm{Y}$ & & $\mathrm{Y}$ & & $\mathrm{Y}$ & $\mathrm{Y}$ & $\mathrm{Y}$ & & & & $\mathrm{Y}$ & & & & $\mathrm{Y}$ & $\mathrm{Y}$ & & $\mathrm{Y} / \mathrm{Y}$ & $\mathrm{Y}$ & $\mathrm{Y}$ & & $\mathrm{Y}$ \\
\hline Chamaecyparis lawsoniana & & & & & & & & & & & & & & & & & & & & & & & & & $\mathrm{Y} / \mathrm{Y}$ & & & & $\mathrm{Y}$ \\
\hline Chamaecyparis obtusa & & & & & & $\mathrm{Y}$ & & $\mathrm{Y}$ & & & & & & & & & & & & & & $\mathrm{Y}$ & $\mathrm{Y}$ & & $\mathrm{Y} / \mathrm{Y}$ & $\mathrm{Y}$ & $\mathrm{Y}$ & & \\
\hline Chamaecyparis pisifera & & & & & & $\mathrm{Y}$ & & $\mathrm{Y}$ & & & & & & & $\mathrm{Y}$ & & & & $\mathrm{Y}$ & $\mathrm{Y}$ & & & $\mathrm{Y}$ & & $\mathrm{Y} / \mathrm{Y}$ & $\mathrm{Y}$ & $\mathrm{Y}$ & & $\mathrm{Y}$ \\
\hline Chamaecyparis spp. & & $\mathrm{Y} /-$ & $\mathrm{Y}$ & $\mathrm{Y}$ & & & & & & & & $\mathrm{Y}$ & & $\mathrm{Y}$ & & & & $\mathrm{Y}$ & & & & $Y^{11}$ & & & & & & & \\
\hline
\end{tabular}




\begin{tabular}{|c|c|c|c|c|c|c|c|c|c|c|c|c|c|c|c|c|c|c|c|c|c|c|c|c|c|c|c|c|c|}
\hline Scientific name & 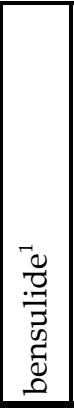 & 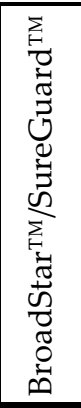 & 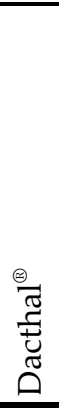 & 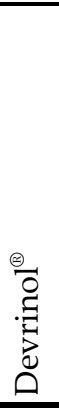 & 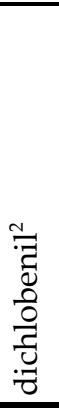 & 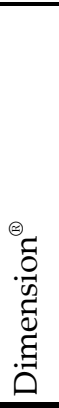 & 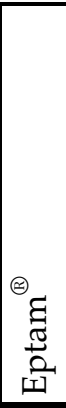 & 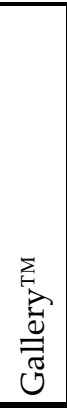 & $\begin{array}{l}\otimes_{0} \\
\infty \\
\Xi \\
\Xi\end{array}$ & 这 & 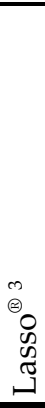 & 量 & 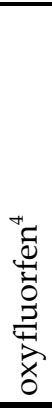 & 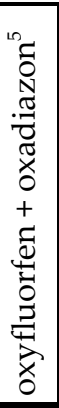 & 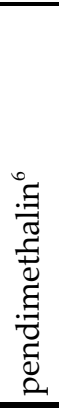 & 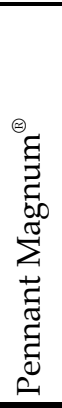 & 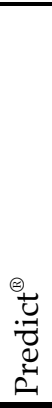 & 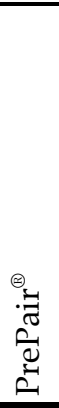 & 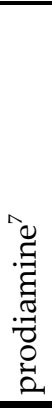 & 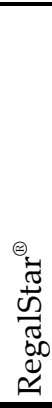 & 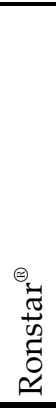 & 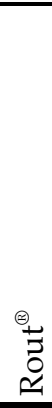 & 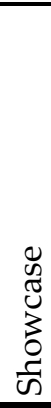 & 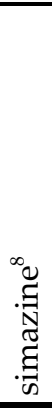 & 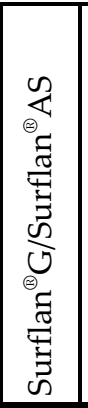 & 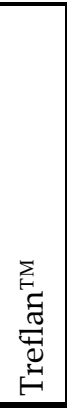 & 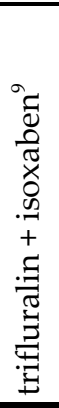 & 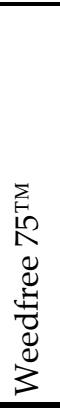 & $\stackrel{\oplus}{\times}$ \\
\hline Chamaedorea cataractarum & & & & & & & & & & & & & & & & & & & & & & & $\mathrm{Y}$ & & & & & & \\
\hline Chamaedorea elegans & & & & & & & & $\mathrm{Y}$ & & & & $\mathrm{Y}$ & & $\mathrm{Y}$ & & & & $\mathrm{Y}$ & & & & & $\mathrm{Y}$ & & $\mathrm{Y} / \mathrm{Y}$ & $\mathrm{Y}$ & $\mathrm{Y}$ & & $\mathrm{Y}$ \\
\hline Chamaedorea erumpens & & & & & & & & & & & & & & & & & & $\mathrm{Y}$ & & & & & & & & & & & \\
\hline Chamaedorea spp. & & $\mathrm{Y} /-$ & & & & & & & & & & & & & & & & & & & & & & & & & & & \\
\hline Chamaerops humilis & & & & & & & & $\mathrm{Y}$ & & & & & & & & & & & & & & & & & & & & & \\
\hline Chrysalidocarpus lutescens & & & & & & & & $\mathrm{Y}$ & & & & & & & & & & & & & & & & & $\mathrm{Y} / \mathrm{Y}$ & $\mathrm{Y}$ & $\mathrm{Y}$ & & $\mathrm{Y}$ \\
\hline Citrus spp. & & $\mathrm{Y} / \mathrm{Y}$ & & $\mathrm{Y}$ & & & & & & & & & & & & $\mathrm{Y}$ & $\mathrm{Y}$ & $\mathrm{Y}$ & $\mathrm{Y}$ & & $Y^{11}$ & & & & $-/ Y$ & & & & \\
\hline Cleyera japonica & & $\mathrm{N} /-$ & & & $\mathrm{Y}$ & & & $\mathrm{Y}$ & & & & & & & & & & & $\mathrm{Y}$ & & $\mathrm{Y}$ & & & & $\mathrm{Y} / \mathrm{Y}$ & $\mathrm{Y}$ & $\mathrm{Y}$ & & $\mathrm{Y}$ \\
\hline Codiaeum variegatum & & & & & & & & & & & & & & & & & & $\mathrm{Y}$ & & & & $\mathrm{Y}$ & & & & & & & \\
\hline Cornus florida & & $Y / Y^{11}$ & $\mathrm{Y}$ & $\mathrm{Y}$ & $\mathrm{Y}$ & $\mathrm{Y}$ & & $\mathrm{Y}$ & & $\mathrm{Y}$ & $\mathrm{Y}$ & $\mathrm{Y}$ & $\mathrm{Y}$ & $\mathrm{Y}$ & $\mathrm{Y}$ & $\mathrm{Y}$ & $\mathrm{Y}$ & $\mathrm{Y}$ & $\mathrm{Y}$ & $\mathrm{Y}$ & $\mathrm{Y}$ & $\mathrm{Y}$ & $\mathrm{Y}$ & $\mathrm{Y}$ & $\mathrm{Y} / \mathrm{Y}$ & $\mathrm{Y}$ & $\mathrm{Y}$ & & $\mathrm{Y}$ \\
\hline Cornus kousa & & & $\mathrm{Y}$ & $\mathrm{Y}$ & $\mathrm{Y}$ & & & & & $\mathrm{Y}$ & $\mathrm{Y}$ & $\mathrm{Y}$ & & $\mathrm{Y}$ & $\mathrm{Y}$ & $\mathrm{Y}$ & $\mathrm{Y}$ & $\mathrm{Y}$ & & & $\mathrm{Y}$ & & & $\mathrm{Y}$ & $\mathrm{Y} / \mathrm{Y}$ & $\mathrm{Y}$ & & & $\mathrm{Y}$ \\
\hline Cornus stolonifera & & & $\mathrm{Y}$ & $\mathrm{Y}$ & $\mathrm{Y}$ & $\mathrm{Y}$ & & & & $\mathrm{Y}$ & $\mathrm{Y}$ & $\mathrm{Y}$ & & $\mathrm{Y}$ & & $\mathrm{Y}$ & $\mathrm{Y}$ & $\mathrm{Y}$ & $\mathrm{Y}$ & & $\mathrm{Y}$ & & $\mathrm{Y}$ & $\mathrm{Y}$ & $\mathrm{Y} / \mathrm{Y}$ & $\mathrm{Y}$ & & & $\mathrm{Y}$ \\
\hline Cotoneaster apiculatus & & & & & & $\mathrm{Y}$ & & $\mathrm{Y}$ & & & & & & & & & & & $\mathrm{Y}$ & & & & $\mathrm{Y}$ & & $\mathrm{Y} / \mathrm{Y}$ & $\mathrm{Y}$ & $\mathrm{Y}$ & & $\mathrm{Y}$ \\
\hline Cotoneaster spp. & & $\mathrm{Y} /-$ & $\mathrm{Y}$ & $\mathrm{Y}$ & $\mathrm{Y}$ & & & $Y^{11}$ & & $\mathrm{Y}$ & $\mathrm{Y}$ & $\mathrm{Y}$ & & $\mathrm{Y}$ & $\mathrm{Y}$ & $\mathrm{Y}$ & & $\mathrm{Y}^{11}$ & $Y^{11}$ & $\mathrm{Y}$ & $\mathrm{Y}$ & $\mathrm{Y}^{11}$ & $\mathrm{Y}$ & $\mathrm{Y}$ & $\mathrm{Y} / \mathrm{Y}^{11}$ & $\mathrm{Y}^{11}$ & $\mathrm{Y}^{11}$ & & $Y^{11}$ \\
\hline$\times$ Cupressocyparis leylandii & & & $\mathrm{Y}$ & $\mathrm{Y}$ & & $\mathrm{Y}$ & & $\mathrm{Y}$ & & & & & & & $\mathrm{Y}$ & & & $\mathrm{Y}$ & & & $\mathrm{Y}$ & & & & & & & & \\
\hline Cupressus arizonica & & & & & & & & $\mathrm{Y}$ & & & & & & & & & & & & & & & $\mathrm{Y}$ & & $\mathrm{Y} / \mathrm{Y}$ & $\mathrm{Y}$ & $\mathrm{Y}$ & & $\mathrm{Y}$ \\
\hline \multicolumn{30}{|l|}{$\begin{array}{l}\text { Cupressus glabra (see } C \text {. } \\
\text { arizonica) }\end{array}$} \\
\hline Cupressus sempervirens & & $\mathrm{Y} /-$ & & & & $\mathrm{Y}$ & & $\mathrm{Y}$ & & & & & & & & & & & $\mathrm{Y}$ & & & Y & $\mathrm{Y}$ & & $\mathrm{Y} / \mathrm{Y}$ & & $\mathrm{Y}$ & & \\
\hline Cupressus spp. & $\mathrm{Y}^{11}$ & & $\mathrm{Y}$ & $\mathrm{Y}$ & $\mathrm{Y}^{11}$ & & & $\mathrm{Y}^{11}$ & & & & $\mathrm{Y}$ & & $\mathrm{Y}$ & & & & $\mathrm{Y}$ & & $\mathrm{Y}$ & $\mathrm{Y}$ & & & & $\mathrm{Y} / \mathrm{Y}^{11}$ & $\mathrm{Y}^{11}$ & $\mathrm{Y}^{11}$ & & \\
\hline Cycas revoluta & & & & & & $\mathrm{Y}$ & & $\mathrm{Y}$ & & & & & & & & & & & & & & & & & & $\mathrm{Y}$ & $\mathrm{Y}$ & & \\
\hline Deutzia spp. & & $\mathrm{N} /-$ & $\mathrm{Y}$ & & $\mathrm{Y}$ & & & $Y^{11}$ & & & & & & & $\mathrm{Y}^{11}$ & & & & & & $\mathrm{Y}^{11}$ & & & & $\mathrm{Y} / \mathrm{Y}^{11}$ & $\mathrm{Y}$ & $\mathrm{Y}^{11}$ & & $\mathrm{Y}^{11}$ \\
\hline Elaeagnus angustifolia & & & & & $\mathrm{Y}$ & & & $\mathrm{Y}$ & & & & & $\mathrm{Y}$ & & & $\mathrm{Y}$ & & & & & $\mathrm{Y}$ & & & $\mathrm{Y}$ & $\mathrm{Y} / \mathrm{Y}$ & $\mathrm{Y}$ & $\mathrm{Y}$ & & $\mathrm{Y}$ \\
\hline Elaeagnus pungens & & & $\mathrm{Y}$ & & & & & $\mathrm{Y}$ & & & & & & & $Y^{11}$ & $\mathrm{Y}$ & & & $\mathrm{Y}$ & & & $\mathrm{Y}^{11}$ & $\mathrm{Y}$ & & & $\mathrm{Y}$ & $\mathrm{Y}$ & & \\
\hline
\end{tabular}




\begin{tabular}{|c|c|c|c|c|c|c|c|c|c|c|c|c|c|c|c|c|c|c|c|c|c|c|c|c|c|c|c|c|c|}
\hline Scientific name & 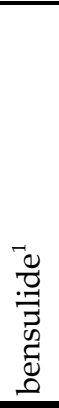 & 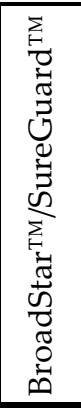 & 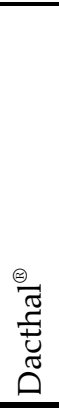 & 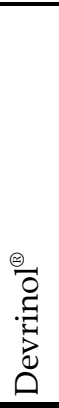 & 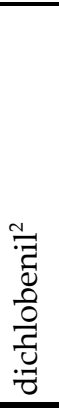 & 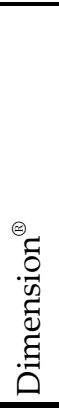 & 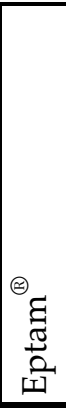 & 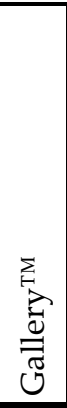 & $\begin{array}{l}\Theta_{0} \\
\infty \\
\Xi \\
\Xi\end{array}$ & $\begin{array}{l}\oplus_{0} \\
\stackrel{0}{\vec{U}} \\
\underline{y}\end{array}$ & 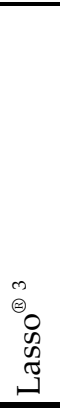 & $\begin{array}{l}\stackrel{\otimes}{\tilde{Z}} \\
\stackrel{1}{0} \\
0\end{array}$ & 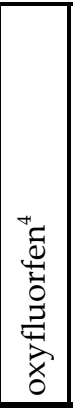 & 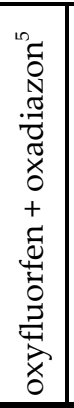 & 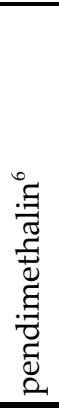 & 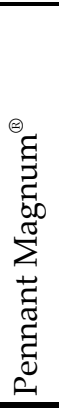 & 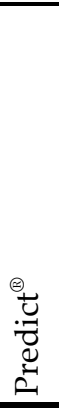 & 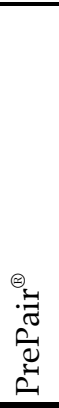 & 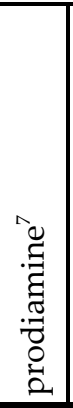 & 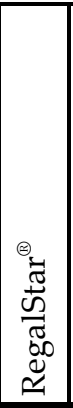 & 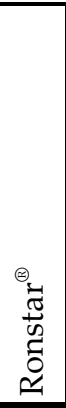 & $\begin{array}{l}\stackrel{\otimes}{+} \\
\stackrel{+}{Z} \\
\stackrel{2}{\simeq}\end{array}$ & 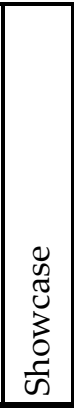 & 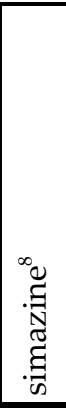 & 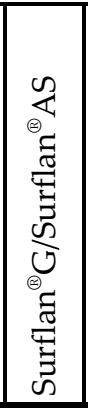 & 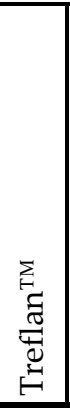 & 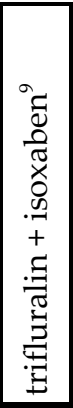 & 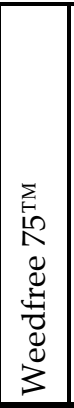 & $\begin{array}{l} \\
\stackrel{\oplus}{\times}\end{array}$ \\
\hline Elaeagnus spp. & & $\mathrm{Y} /-$ & & & & & & & & & & & & & & & & & & & & & & & & & & & \\
\hline Eriobotrya japonica & & & & & & & & & & & & & & & & & & & & & & $\mathrm{Y}$ & & & & & & & \\
\hline Escallonia spp. & & & & & & & & & & & & $\mathrm{Y}$ & & & & & & & & & & & & & & & & & \\
\hline Eucalyptus camaldulensis & & $\mathrm{Y} /-$ & & & & & & & & & & & & & & & & & & & & & & & $\mathrm{Y} / \mathrm{Y}$ & & & & \\
\hline Eucalyptus cinerea & & $\mathrm{Y} /-$ & & & & & & $\mathrm{Y}$ & & & & & & & & & & & & & & & & $\mathrm{Y}$ & $\mathrm{Y} / \mathrm{Y}$ & $\mathrm{Y}$ & $\mathrm{Y}$ & & $\mathrm{Y}$ \\
\hline Eucalyptus citriodora & & & & & & $\mathrm{Y}$ & & & & & & & & & & & & & & & & & & & & & & & \\
\hline Eucalyptus spp. & & $-/ Y$ & & $\mathrm{Y}$ & & & & $Y^{11}$ & & & & & $\mathrm{Y}^{11}$ & & & & & $\mathrm{Y}$ & & & $\mathrm{Y}$ & & & $\mathrm{Y}$ & $Y / Y^{11}$ & $\mathrm{Y}^{11}$ & $\mathrm{Y}^{11}$ & & $Y^{11}$ \\
\hline Euonymus fortunei & & & & & & $\mathrm{Y}^{11}$ & & $Y^{11}$ & & & & & & & $\mathrm{Y}$ & & & $\mathrm{Y}$ & $\bar{Y}$ & $\mathrm{Y}^{11}$ & $\mathrm{Y}$ & $\mathrm{Y}^{11}$ & $\mathrm{Y}$ & & $Y / Y^{11}$ & $\mathrm{Y}^{11}$ & $\mathrm{Y}^{11}$ & & $\mathrm{Y}^{11}$ \\
\hline Euonymus japonica & & & & & & & & $Y^{11}$ & & $\mathrm{Y}$ & $\mathrm{Y}$ & & & & $\mathrm{Y}$ & & & & $\bar{Y}$ & & & $\mathrm{~N}^{11}$ & $\mathrm{Y}$ & & $\mathrm{Y} / \mathrm{Y}^{11}$ & $\mathrm{Y}^{11}$ & $\mathrm{Y}^{11}$ & & $\mathrm{Y}^{11}$ \\
\hline Euonymous kiautshovicus & & & & & & & & $\mathrm{Y}$ & & & & & & & & & & & $\mathrm{Y}$ & & $\mathrm{Y}$ & $\mathrm{Y}^{11}$ & $\mathrm{Y}$ & & $\mathrm{Y} / \mathrm{Y}$ & $\mathrm{Y}$ & $\mathrm{Y}$ & & $\mathrm{Y}$ \\
\hline Euonymous spp. & & $\mathrm{Y}^{11 /-}$ & $\mathrm{Y}$ & $\mathrm{Y}$ & $\mathrm{Y}$ & & & $Y^{11}$ & & & & $\mathrm{Y}^{11}$ & & $\mathrm{Y}^{11}$ & $\mathrm{Y}^{11}$ & $\mathrm{Y}$ & & & & & & $Y^{11}$ & $\mathrm{Y}$ & & $Y / Y^{11}$ & $\mathrm{Y}^{11}$ & $\mathrm{Y}^{11}$ & & $Y^{11}$ \\
\hline$\times$ Fatshedera lizei & & & & & & & & & & & & & & & & & & & & & & & & & $\mathrm{Y} / \mathrm{Y}$ & & & & $\mathrm{Y}$ \\
\hline Fatsia japonica & & & & & & & & $\mathrm{Y}$ & & & & & & & & & & & & & & & & & $-/ Y$ & & $\mathrm{Y}$ & & \\
\hline Ficus benjamina & & $\mathrm{Y} /-$ & & & & & & $\mathrm{Y}$ & & & & & & & & & & & & & & $\mathrm{Y}$ & $\mathrm{Y}$ & & $\mathrm{Y} / \mathrm{Y}$ & $\mathrm{Y}$ & & & $\mathrm{Y}$ \\
\hline Ficus lyrata & & & & & & & & & & & & & & & & & & & & & & $\bar{Y}$ & & & & & & & \\
\hline Ficus spp. & & & & $\mathrm{Y}$ & & & & & & & & & & & & $\mathrm{Y}$ & & & & & & $\mathrm{Y}^{11}$ & & & & & & & \\
\hline Forsythia $\times$ intermedia & & & & & & $\mathrm{Y}$ & & $\mathrm{Y}$ & & & & & & & $\mathrm{Y}$ & & & & $\mathrm{Y}$ & $\mathrm{Y}$ & $\mathrm{Y}$ & $\mathrm{Y}^{11}$ & $\mathrm{Y}$ & & $\mathrm{Y} / \mathrm{Y}$ & & $\mathrm{Y}$ & & $\mathrm{Y}$ \\
\hline Forsythia suspensa & & $\mathrm{Y} /-$ & & & & & & & & & & & & & & & & & & & & & & & & & & & \\
\hline Forsythia spp. & & & & $\mathrm{Y}$ & $\mathrm{Y}$ & & & & & $\mathrm{Y}$ & & $\mathrm{Y}$ & & $\mathrm{Y}$ & & $\mathrm{Y}$ & & $\mathrm{Y}$ & $\mathrm{Y}^{11}$ & & & & & & & $\mathrm{Y}$ & $\mathrm{Y}^{11}$ & & \\
\hline Fraxinus spp. & & $\mathrm{Y} / \mathrm{Y}$ & $\mathrm{Y}$ & $\mathrm{Y}$ & $\mathrm{Y}$ & $\mathrm{Y}$ & & $Y^{11}$ & & $\mathrm{Y}$ & & & $\mathrm{Y}^{11}$ & & $\mathrm{Y}$ & $\mathrm{Y}$ & & $\mathrm{Y}$ & & & $Y^{11}$ & & & & $\mathrm{Y} / \mathrm{Y}$ & $\mathrm{Y}^{11}$ & $\mathrm{Y}^{11}$ & & $\mathrm{Y}$ \\
\hline Gardenia jasminoides & & & & & & $\mathrm{Y}$ & & $\mathrm{Y}$ & $\mathrm{Y}$ & & & & & & $\mathrm{Y}$ & $\mathrm{Y}$ & & & $\mathrm{Y}$ & $\mathrm{Y}$ & $\mathrm{Y}$ & $\mathrm{Y}$ & & & $\mathrm{Y} / \mathrm{Y}$ & $\mathrm{Y}$ & $\mathrm{Y}$ & & $\mathrm{Y}$ \\
\hline Gardenia spp. & & $\mathrm{Y} /-$ & & $\mathrm{Y}$ & $\mathrm{Y}$ & & & & & & & $Y$ & & $\mathrm{Y}$ & & $\mathrm{Y}$ & & $\mathrm{Y}$ & & & & & & & & & & & \\
\hline Ginko biloba & & $\mathrm{Y} / \mathrm{Y}$ & & & & & & $\mathrm{Y}$ & & $\mathrm{Y}$ & & & & & $\mathrm{Y}$ & $\mathrm{Y}$ & & & & & $\mathrm{Y}$ & & & & $\mathrm{Y} / \mathrm{Y}$ & $Y$ & $\mathrm{Y}$ & & $\mathrm{Y}$ \\
\hline Hibiscus rosa-sinensis & & & & $\mathrm{Y}$ & & $\mathrm{Y}$ & & $\mathrm{Y}$ & & & & & & & & $\mathrm{Y}$ & & $\mathrm{Y}$ & $\mathrm{Y}$ & & $\mathrm{Y}$ & $\mathrm{Y}^{11}$ & $Y$ & & $\mathrm{Y} / \mathrm{Y}$ & $Y$ & $\mathrm{Y}$ & & $\mathrm{Y}$ \\
\hline
\end{tabular}




\begin{tabular}{|c|c|c|c|c|c|c|c|c|c|c|c|c|c|c|c|c|c|c|c|c|c|c|c|c|c|c|c|c|c|}
\hline Scientific name & 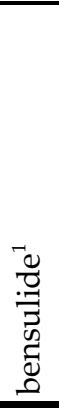 & 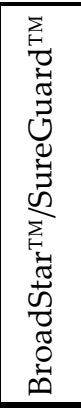 & 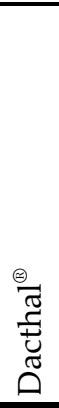 & 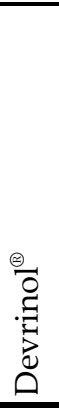 & 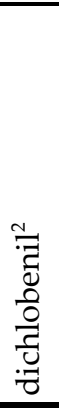 & 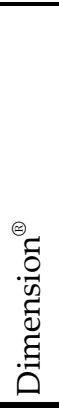 & 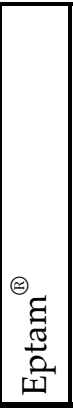 & 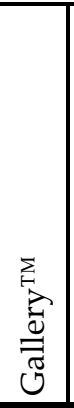 & 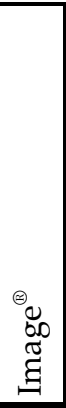 & 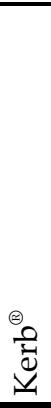 & 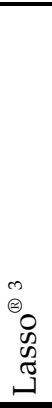 & $\stackrel{\oplus}{\stackrel{\oplus}{0}}$ & 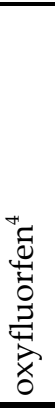 & 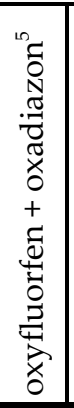 & 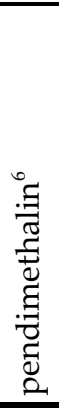 & 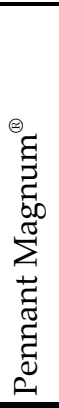 & 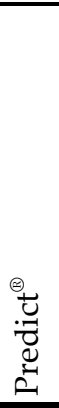 & 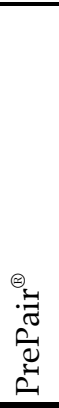 & 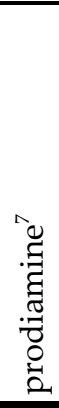 & 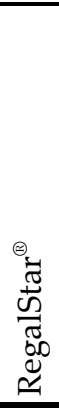 & 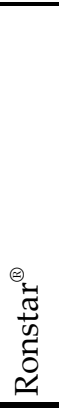 & 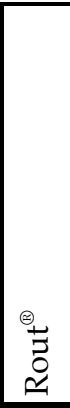 & 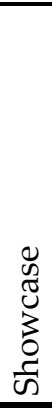 & 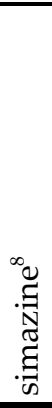 & 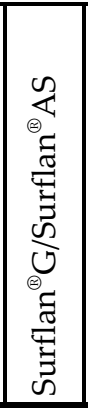 & 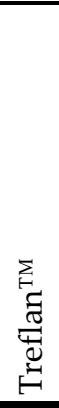 & 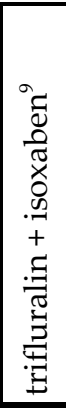 & 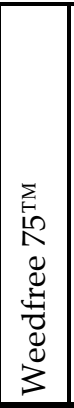 & 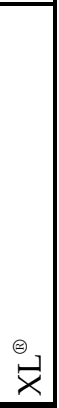 \\
\hline Hibiscus syriacus & & & & $\mathrm{Y}$ & & $Y^{11}$ & & $\mathrm{Y}$ & & & & & & & $\mathrm{Y}$ & $\mathrm{Y}$ & & $\mathrm{Y}^{11}$ & $\mathrm{Y}$ & & $\mathrm{Y}^{11}$ & & & & $\mathrm{Y} / \mathrm{Y}$ & $\mathrm{Y}$ & $\mathrm{Y}$ & & $\mathrm{Y}$ \\
\hline Hydrangea macrophylla & & & & & & & & & & & & & & & $\mathrm{Y}^{11}$ & & & & $\mathrm{Y}$ & & & & & & & & & & \\
\hline Hydrangea spp. & & $\mathrm{N} /-$ & $\mathrm{Y}$ & & & & & $\mathrm{N}$ & & & & $\mathrm{Y}$ & & $\mathrm{Y}$ & & $\mathrm{Y}$ & & & & & & $\mathrm{N}^{11}$ & & & & & $\mathrm{~N}$ & & \\
\hline Ilex attenuata 'Savannah' & & & & & $\mathrm{Y}$ & $\mathrm{Y}$ & & $\mathrm{Y}^{11}$ & & & & & & & $\mathrm{Y}$ & & $\mathrm{Y}$ & & & & & & $\mathrm{Y}$ & $\mathrm{Y}$ & & & $\mathrm{Y}$ & & \\
\hline Ilex cornuta & & & & & $\mathrm{Y}$ & $\mathrm{Y}$ & & $\mathrm{Y}^{11}$ & $\mathrm{Y}^{11}$ & & & & & & $\mathrm{Y}$ & & $\mathrm{Y}^{11}$ & & $\mathrm{Y}$ & & & $Y^{11}$ & $\mathrm{Y}$ & $\mathrm{Y}$ & $Y / Y^{11}$ & & $Y^{11}$ & & $Y^{11}$ \\
\hline Ilex crenata & & $\mathrm{N}^{11} /-$ & & & $\mathrm{N}$ & $\mathrm{Y}$ & & $\mathrm{Y}^{11}$ & $\mathrm{Y}^{11}$ & & & & & & $\mathrm{Y}$ & & $\mathrm{Y}^{11}$ & & $\mathrm{Y}$ & & & $Y^{11}$ & $\mathrm{Y}$ & $\mathrm{Y}$ & $Y / Y^{11}$ & & $Y^{11}$ & & $Y^{11}$ \\
\hline Ilex glabra & & & & & $\mathrm{Y}$ & & & $\mathrm{Y}^{11}$ & & & & & & & & & & & & & & & $\mathrm{Y}$ & & $Y / Y^{11}$ & & $Y^{11}$ & & \\
\hline Ilex opaca & & & & & $\mathrm{Y}$ & & & & & & & & & & & & $\mathrm{Y}^{11}$ & & $\mathrm{Y}$ & & & & & $\mathrm{Y}$ & & & & & \\
\hline Ilex vomitoria & & & & & $\mathrm{N}$ & $\mathrm{Y}$ & & $\mathrm{Y}^{11}$ & $\mathrm{Y}^{11}$ & & & & & & $\mathrm{Y}$ & & & & $\mathrm{Y}$ & & & $\mathrm{Y}$ & $\mathrm{Y}$ & $\mathrm{Y}$ & $\mathrm{Y} / \mathrm{Y}^{11}$ & & $Y^{11}$ & & $Y^{11}$ \\
\hline Ilex $\times$ 'Nellie Stevens' & & & & & $\mathrm{Y}$ & $\mathrm{Y}$ & & & & & & & & & $\mathrm{Y}$ & & $\mathrm{Y}$ & & $\mathrm{Y}$ & & & & & $\mathrm{Y}$ & & & & & \\
\hline Ilex spp. & $\mathrm{Y}$ & $\mathrm{Y} /-$ & $\mathrm{Y}$ & $\mathrm{Y}$ & $\mathrm{Y}^{11}$ & $Y^{11}$ & & $\mathrm{Y}^{11}$ & & $\mathrm{Y}$ & $\mathrm{Y}$ & $\mathrm{Y}$ & & $\mathrm{Y}^{11}$ & & $\mathrm{Y}$ & & $\mathrm{Y}$ & $Y^{11}$ & $Y^{11}$ & $\mathrm{Y}$ & & & $\mathrm{Y}$ & $Y / Y^{11}$ & $\mathrm{Y}$ & $Y^{11}$ & & $Y^{11}$ \\
\hline Illicium anisatum & & & & & & & & $\mathrm{Y}$ & & & & & & & & $\mathrm{Y}$ & & & & & & & & & & & Y & & \\
\hline Illicium floridanum & & $\mathrm{Y} /-$ & & & & & & $\mathrm{Y}$ & & & & & & & & $\mathrm{Y}$ & & & & & & & & & & $\mathrm{Y}$ & $\mathrm{Y}$ & & \\
\hline Illicium parviflorum & & & & & & & & & & & & & & & $\mathrm{Y}$ & $\mathrm{Y}$ & & & & & & & & & & & & & \\
\hline Itea ilicifolia & & $\mathrm{Y}^{11 /-}$ & & & & & & $\mathrm{Y}$ & & & & & & & & & & & & & & & & & & $\mathrm{Y}$ & $\mathrm{Y}$ & & \\
\hline Ixora coccinea & & & & & & & & $\mathrm{Y}$ & $\mathrm{Y}$ & & & & & & & & & & & & & & & & & $\mathrm{Y}$ & $\mathrm{Y}$ & & \\
\hline Juniperus chinensis & & $-/ Y$ & & & & $Y^{11}$ & & $\mathrm{Y}^{11}$ & $\mathrm{Y}^{11}$ & & & & $\mathrm{Y}$ & & $\mathrm{Y}$ & & & & & $\mathrm{Y}$ & & $\mathrm{Y}^{11}$ & $\mathrm{Y}$ & & $\mathrm{Y} / \mathrm{Y}$ & & $Y^{11}$ & & \\
\hline Juniperus conferta & & & & & & & & $\mathrm{Y}^{11}$ & $\mathrm{Y}^{11}$ & & & & & & $\mathrm{Y}$ & & & & & & & $\mathrm{Y}^{11}$ & $\mathrm{Y}$ & & $\mathrm{Y} / \mathrm{Y}$ & & $Y^{11}$ & & \\
\hline Juniperus horizontalis & & $-/ Y$ & & & & $\mathrm{Y}^{11}$ & & $\mathrm{Y}^{11}$ & $\mathrm{Y}^{11}$ & & & & $\mathrm{Y}$ & & $\mathrm{Y}$ & & $\mathrm{Y}^{11}$ & & & & & $\mathrm{Y}^{11}$ & $\mathrm{Y}$ & & $\mathrm{Y} / \mathrm{Y}$ & & $Y^{11}$ & & \\
\hline Juniperus procumbens & & & & & & & & $\mathrm{Y}^{11}$ & & & & & $\mathrm{Y}$ & & & & & & & & & & $\mathrm{Y}$ & & $\mathrm{Y} / \mathrm{Y}$ & & $Y^{11}$ & & \\
\hline Juniperus sabina & & & & & & $Y^{11}$ & & $\mathrm{Y}^{11}$ & & & & & $\mathrm{Y}$ & & & & & & & & & $\mathrm{Y}^{11}$ & $\mathrm{Y}$ & & $\mathrm{Y} / \mathrm{Y}$ & & $Y^{11}$ & & \\
\hline \multicolumn{30}{|l|}{ Juniperus silicicola } \\
\hline Juniperus squamata & & & & & & & & $\mathrm{Y}^{11}$ & $\mathrm{Y}^{11}$ & & & & & & & & & & & & & $\mathrm{Y}^{11}$ & $\mathrm{Y}$ & & $\mathrm{Y} / \mathrm{Y}$ & & $\mathrm{Y}^{11}$ & & \\
\hline Juniperus virginiana & $\mathrm{Y}$ & & $\mathrm{Y}$ & $\mathrm{Y}$ & $\mathrm{Y}$ & & & $\mathrm{Y}$ & & $\mathrm{Y}$ & $\mathrm{Y}$ & $\mathrm{Y}$ & $\mathrm{Y}$ & $\mathrm{Y}$ & $\mathrm{Y}$ & $\mathrm{Y}$ & & $\mathrm{Y}$ & & $\mathrm{Y}$ & $\mathrm{Y}$ & & $\mathrm{Y}$ & & $\mathrm{Y} / \mathrm{Y}$ & $\mathrm{Y}$ & $\mathrm{Y}$ & & $\mathrm{Y}$ \\
\hline
\end{tabular}




\begin{tabular}{|c|c|c|c|c|c|c|c|c|c|c|c|c|c|c|c|c|c|c|c|c|c|c|c|c|c|c|c|c|c|}
\hline Scientific name & 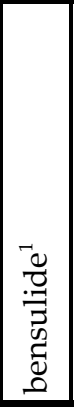 & 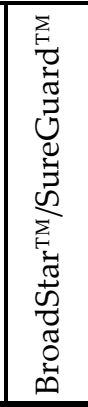 & 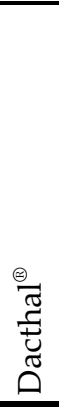 & 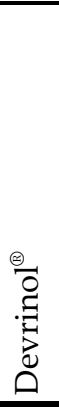 & 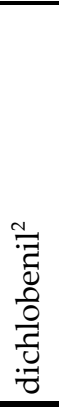 & 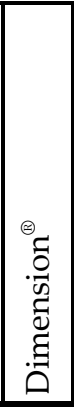 & 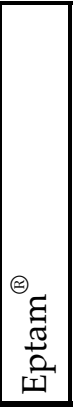 & 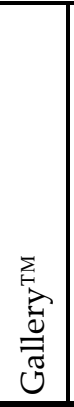 & $\begin{array}{l}\Theta_{0} \\
\infty \\
\Xi \\
\Xi \\
\Xi\end{array}$ & $\begin{array}{l}\Theta_{0} \\
\stackrel{\vec{u}}{\Delta} \\
\end{array}$ & 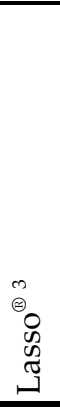 & $\begin{array}{l}\stackrel{\otimes}{\tilde{I}} \\
\tilde{0} \\
0\end{array}$ & 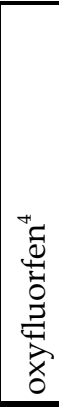 & 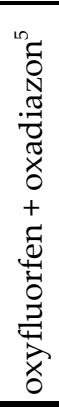 & 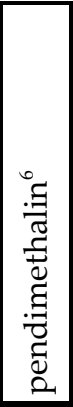 & 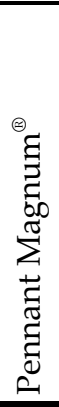 & 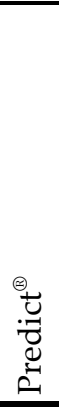 & 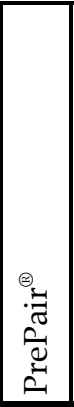 & 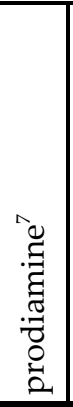 & 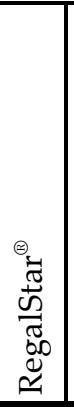 & 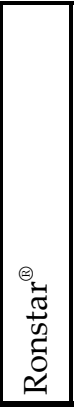 & 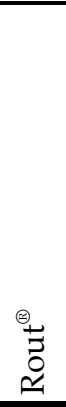 & \begin{tabular}{|l}
$\tilde{y}$ \\
$\tilde{J}$ \\
$\tilde{J}$ \\
3 \\
0 \\
$\tilde{E}$ \\
\end{tabular} & 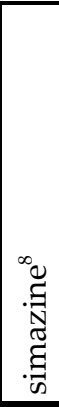 & 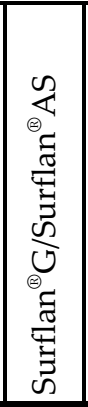 & 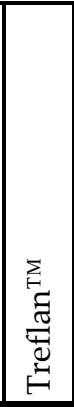 & 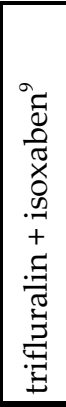 & 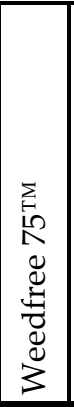 & $\stackrel{\Theta}{P}_{\vec{X}}$ \\
\hline Juniperus spp. & $\mathrm{Y}$ & $\mathrm{Y} /-$ & $\mathrm{Y}$ & $\mathrm{Y}$ & $\mathrm{Y}$ & $\mathrm{Y}^{11}$ & & $\mathrm{Y}^{11}$ & & $\mathrm{Y}$ & $\mathrm{Y}$ & $\mathrm{Y}$ & $Y^{11}$ & $\mathrm{Y}$ & $\mathrm{Y}$ & $\mathrm{Y}$ & $Y^{11}$ & $\mathrm{Y}$ & $\mathrm{Y}^{11}$ & & $\mathrm{Y}$ & $Y^{11}$ & $\mathrm{Y}^{11}$ & $\mathrm{Y}^{11}$ & $Y / Y^{11}$ & $\mathrm{Y}$ & $\mathrm{Y}^{11}$ & & $\mathrm{Y}$ \\
\hline Lagerstroemia indica & & $\mathrm{N}^{11} / \mathrm{Y}$ & & $\mathrm{Y}$ & & $\mathrm{Y}^{11}$ & & $\mathrm{Y}$ & $\mathrm{Y}$ & & & & $\mathrm{Y}$ & & $\mathrm{Y}$ & $\mathrm{Y}$ & $\mathrm{Y}$ & $\mathrm{Y}^{11}$ & $\mathrm{Y}$ & & $\mathrm{Y}^{11}$ & & & & $\mathrm{Y} / \mathrm{Y}$ & & $\mathrm{Y}$ & & $\mathrm{Y}$ \\
\hline Lagerstroemia spp. & & $\mathrm{Y}^{11} /-$ & & & & & & & & & & & & & & & & & & & & & & & & & & & \\
\hline Leptospermum scoparium & & & & & & & & $\mathrm{Y}$ & & & & & & & & & & & & & & & & & & & & & \\
\hline Leucophyllum frutescens & & & & & & & & $\mathrm{Y}$ & & & & & & & & & & & & & & & & & & & & & \\
\hline Leucothoe axillaries & & & & $\mathrm{Y}$ & $\mathrm{Y}$ & & & $\mathrm{Y}$ & & & & & & & $\mathrm{Y}$ & $\mathrm{Y}$ & & $Y$ & & & $\mathrm{Y}$ & & & $\mathrm{Y}$ & $\mathrm{Y} / \mathrm{Y}$ & $\mathrm{Y}$ & $\mathrm{Y}$ & & $\mathrm{Y}$ \\
\hline Leucothoe fontanesiana & & & & $\mathrm{Y}$ & $\mathrm{Y}$ & $\mathrm{Y}$ & & $\mathrm{Y}$ & & & & & & & $\mathrm{Y}$ & $\mathrm{Y}$ & & $\mathrm{Y}$ & & & $\mathrm{Y}$ & & & $\mathrm{Y}$ & $\mathrm{Y} / \mathrm{Y}$ & $\mathrm{Y}$ & $\mathrm{Y}$ & & $\mathrm{Y}$ \\
\hline Ligustrum japonicum & & & & & & $\bar{Y}$ & & $\mathrm{Y}$ & & & & & & & $\mathrm{Y}$ & & & & $\mathrm{Y}$ & & & $\mathrm{Y}$ & $\mathrm{Y}$ & & $\mathrm{Y} / \mathrm{Y}$ & & $\mathrm{Y}$ & & $\mathrm{Y}$ \\
\hline Ligustrum lucidum & $\mathrm{Y}$ & & $\mathrm{Y}$ & $\mathrm{Y}$ & $\mathrm{Y}$ & $Y$ & & $\mathrm{Y}$ & & $\mathrm{Y}$ & & $Y$ & & $\mathrm{Y}$ & $Y$ & $\mathrm{Y}$ & & & $\mathrm{Y}$ & & $\mathrm{Y}$ & $\mathrm{Y}$ & $\mathrm{Y}$ & & $\mathrm{Y} / \mathrm{Y}$ & $\mathrm{Y}$ & $\mathrm{Y}$ & & $\mathrm{Y}$ \\
\hline L. sinense 'Variegated' & & & & & & & & & & & & & & & $\mathrm{Y}$ & & & & $\mathrm{Y}$ & & & $\mathrm{Y}$ & & & & & & & \\
\hline Ligustrum $\times$ vicaryi & & & & & & & & $\mathrm{Y}$ & & & & & & & & & & & & & & $\mathrm{Y}$ & $\mathrm{Y}$ & & $\mathrm{Y} / \mathrm{Y}$ & & $\mathrm{Y}$ & & $\mathrm{Y}$ \\
\hline Ligustrum spp. & $\mathrm{Y}$ & N/- & $\mathrm{Y}$ & $\mathrm{Y}$ & $\mathrm{Y}$ & & & $\mathrm{Y}^{11}$ & $\mathrm{~N}$ & $\mathrm{Y}$ & & $\mathrm{Y}$ & & $\mathrm{Y}$ & $\mathrm{Y}^{11}$ & $\mathrm{Y}$ & & & $\mathrm{Y}^{11}$ & & $\mathrm{Y}$ & & $\mathrm{Y}^{11}$ & & $Y / Y^{11}$ & $\mathrm{Y}$ & $\mathrm{Y}^{11}$ & & $Y^{11}$ \\
\hline Liquidambar styraciflua & & $\mathrm{Y} / \mathrm{Y}$ & & & & $\mathrm{Y}$ & & $\mathrm{Y}$ & & $\mathrm{Y}$ & & & $\mathrm{Y}$ & & $\mathrm{Y}$ & $\mathrm{Y}$ & & & & & & & & & $\mathrm{Y} / \mathrm{Y}$ & $\mathrm{Y}$ & $\mathrm{Y}$ & & $\mathrm{Y}$ \\
\hline Liriodendron tulipifera & & & $\mathrm{Y}$ & & & & & & & $\mathrm{Y}$ & & & $\mathrm{Y}$ & & $\mathrm{Y}$ & $\mathrm{Y}$ & $\mathrm{Y}$ & & & & & & & & & $\mathrm{Y}$ & & & \\
\hline Livistona chinensis & & & & & & & & $\mathrm{Y}$ & & & & & & & & & & & & & & & & & $\mathrm{Y} / \mathrm{Y}$ & $\mathrm{Y}$ & $\mathrm{Y}$ & & $\mathrm{Y}$ \\
\hline Loropetalum chinensis & & & & & & & & & & & & & & & & & & & $\mathrm{Y}^{11}$ & & & $Y^{11}$ & & & & & $\mathrm{Y}$ & & \\
\hline Magnolia grandiflora & & $\mathrm{Y} /-$ & $\mathrm{Y}$ & & $\mathrm{Y}$ & & & $\mathrm{Y}$ & & $\mathrm{Y}$ & & $\mathrm{Y}$ & & $\mathrm{Y}$ & $\mathrm{Y}$ & $\mathrm{Y}$ & $\mathrm{Y}$ & Y & $\mathrm{Y}$ & $\mathrm{Y}$ & $\mathrm{Y}$ & & $\mathrm{Y}$ & & $\mathrm{Y} / \mathrm{Y}$ & $\mathrm{Y}$ & $\mathrm{Y}$ & & $\mathrm{Y}$ \\
\hline Magnolia $\times$ soulangiana & & & $\mathrm{Y}$ & & $\mathrm{Y}$ & & & & & $\mathrm{Y}$ & & $\mathrm{Y}$ & & $\mathrm{Y}$ & $\mathrm{Y}$ & $\mathrm{Y}$ & & & $\mathrm{Y}$ & $\mathrm{Y}$ & $\mathrm{Y}$ & & & & & & & & \\
\hline Magnolia spp. & & & $\mathrm{Y}$ & & $\mathrm{Y}$ & & $\mathrm{Y}$ & $\mathrm{Y}^{11}$ & & $\mathrm{Y}$ & & $\mathrm{Y}$ & & $\mathrm{Y}$ & $\mathrm{Y}^{11}$ & $\mathrm{Y}$ & & & $\mathrm{Y}$ & $\mathrm{Y}$ & $\mathrm{Y}$ & & & & & & & & \\
\hline Mahonia spp. & & $\mathrm{Y} /-$ & & & & & & $\mathrm{Y}^{11}$ & & & & $\mathrm{Y}^{11}$ & & $\mathrm{Y}$ & $\mathrm{Y}^{11}$ & & & & & & $\mathrm{Y}^{11}$ & $Y^{11}$ & $\mathrm{Y}^{11}$ & $\mathrm{Y}$ & $Y / Y^{11}$ & $\mathrm{Y}^{11}$ & $\mathrm{Y}$ & & $Y^{11}$ \\
\hline Morus alba & & $\mathrm{Y} /-$ & & & & & & $\mathrm{Y}$ & & & & & & & & & & & & & & & & & $\mathrm{Y} / \mathrm{Y}$ & $\mathrm{Y}$ & $\mathrm{Y}$ & & $\mathrm{Y}$ \\
\hline Murraya paniculata & & & & & & & & & $\mathrm{Y}$ & $\mathrm{Y}$ & & & & & & & & $\mathrm{Y}$ & & & $\mathrm{Y}$ & & & & & & & & \\
\hline Musa aluminata & & & & & & & & $\mathrm{Y}$ & & & & & & & & & & & & & & & & & & $Y$ & $\mathrm{Y}$ & & \\
\hline Myrica cerifera & & & & & & $Y$ & & $\mathrm{Y}$ & $\mathrm{Y}$ & & & & & & $\mathrm{Y}$ & $\mathrm{Y}$ & & $\mathrm{Y}$ & & & & & & & & $\mathrm{Y}$ & $\mathrm{Y}$ & & \\
\hline
\end{tabular}




\begin{tabular}{|c|c|c|c|c|c|c|c|c|c|c|c|c|c|c|c|c|c|c|c|c|c|c|c|c|c|c|c|c|c|}
\hline Scientific name & 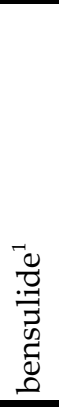 & 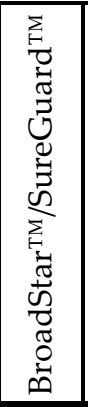 & 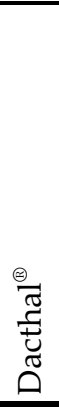 & 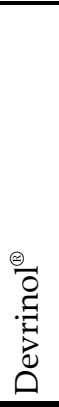 & 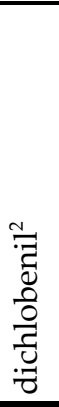 & 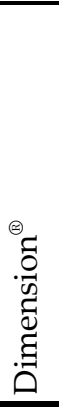 & 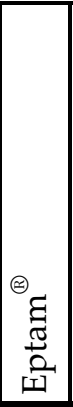 & 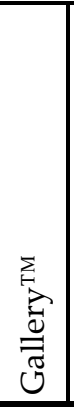 & $\begin{array}{l}\Theta_{0} \\
\stackrel{\infty}{\Xi} \\
\Xi \\
\Xi\end{array}$ & 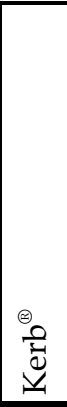 & 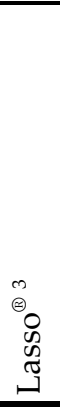 & 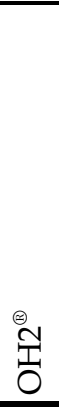 & 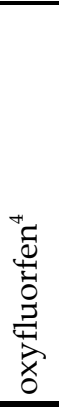 & 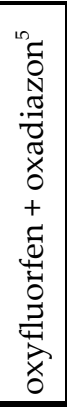 & 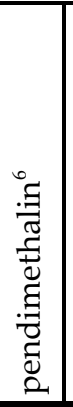 & 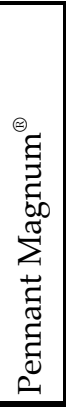 & 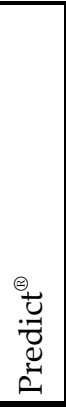 & 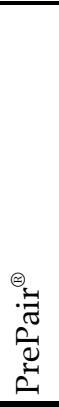 & 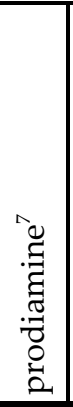 & 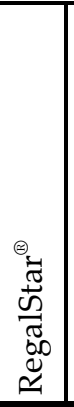 & 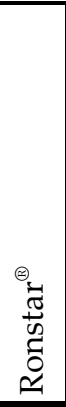 & 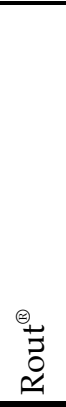 & 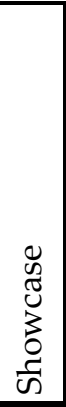 & 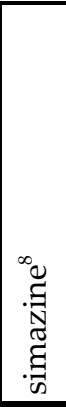 & 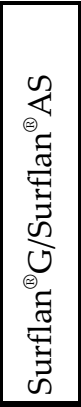 & 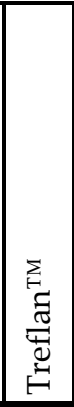 & 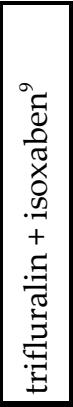 & 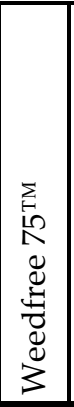 & $\begin{array}{l}\oplus \\
\times \\
\end{array}$ \\
\hline Myrtus communis & & & & & & & & & & & & & & & $\mathrm{Y}$ & & & & & & $\mathrm{Y}$ & & & & $\mathrm{Y} / \mathrm{Y}$ & & & & $\mathrm{Y}$ \\
\hline Nandina domestica & & $\mathrm{N} /-$ & & $\mathrm{Y}$ & $\mathrm{Y}$ & $\mathrm{Y}$ & & $\mathrm{Y}$ & & & & & & & $\mathrm{Y}$ & $\mathrm{Y}$ & & $\mathrm{Y}$ & $\mathrm{Y}$ & & $\mathrm{Y}$ & $\mathrm{Y}$ & & & $\mathrm{Y} / \mathrm{Y}$ & $\mathrm{Y}$ & $\bar{Y}$ & & $\mathrm{Y}$ \\
\hline Nerium oleander & & & & $\mathrm{Y}$ & & $\mathrm{Y}$ & & $\mathrm{Y}^{11}$ & & & & $\mathrm{Y}$ & & $\mathrm{Y}$ & $\mathrm{Y}$ & $\mathrm{Y}$ & & $\mathrm{Y}$ & $\mathrm{Y}$ & $\mathrm{Y}$ & $\mathrm{Y}$ & $\mathrm{Y}$ & $\mathrm{Y}$ & & $\mathrm{Y} / \mathrm{Y}$ & $\mathrm{Y}^{11}$ & $\mathrm{Y}^{11}$ & & $\mathrm{Y}^{11}$ \\
\hline Nerium spp. & & $\mathrm{Y} /-$ & & & & & & & & & & & & & & & & & & & & & & & & & & & \\
\hline Nyssa sylvatica & & & & & & & & & & & & & & & $\mathrm{Y}$ & & & & & & & & & & & $\mathrm{Y}$ & & & \\
\hline Osmanthus fragrans & & $\mathrm{Y} /-$ & & $\mathrm{Y}$ & & & & $\mathrm{Y}$ & & & & & & & $\mathrm{Y}$ & $\mathrm{Y}$ & & $\mathrm{Y}$ & & & & $\mathrm{Y}$ & & $\mathrm{Y}$ & & & & & \\
\hline Osmanthus $\times$ fortunei & & & & $\mathrm{Y}$ & & & & $\mathrm{Y}$ & & & & & & & & $\mathrm{Y}$ & & $\mathrm{Y}$ & & & & & & $\mathrm{Y}$ & & $\mathrm{Y}$ & $\bar{Y}$ & & \\
\hline Osmanthus heterophyllus & & & & $\mathrm{Y}$ & $\mathrm{Y}$ & & & & & & & & & & & $\mathrm{Y}$ & & $\mathrm{Y}$ & $\mathrm{Y}$ & & $\mathrm{Y}$ & $\mathrm{Y}$ & & $\mathrm{Y}$ & $\mathrm{Y} / \mathrm{Y}$ & & & & $\mathrm{Y}$ \\
\hline Phoenix roebelinii & & & & & & & & $\mathrm{Y}$ & & & & & & & & & & & & & & & & & $\mathrm{Y} / \mathrm{Y}$ & $\mathrm{Y}$ & $\mathrm{Y}$ & & $\mathrm{Y}$ \\
\hline Photinia $\times$ fraseri & & & & $\mathrm{Y}$ & $\mathrm{Y}$ & $\mathrm{Y}$ & & $\mathrm{Y}$ & $\mathrm{Y}$ & & & $\mathrm{Y}$ & & $\mathrm{Y}$ & $\mathrm{Y}$ & $\mathrm{Y}$ & & $\mathrm{Y}$ & $\mathrm{Y}$ & $\mathrm{Y}$ & $\mathrm{Y}$ & $\mathrm{Y}$ & $\mathrm{Y}$ & & $\mathrm{Y} / \mathrm{Y}$ & $\mathrm{Y}$ & $\mathrm{Y}$ & & $\mathrm{Y}$ \\
\hline Photinia spp. & & $\mathrm{Y} /-$ & & & & & & & & & & & & & & & & & & & & & & & & & & & \\
\hline Pinus banksiana & & $\mathrm{Y} / \mathrm{Y}$ & & & & & & & & & & & & & & & & & & & & & & & & & & & \\
\hline Pinus bungeana & & Y/- & & & & & & & & & & & & & & & & & & & & & & & & & & & \\
\hline Pinus canariensis & & & & & $\mathrm{Y}$ & & & $\mathrm{Y}$ & & & & & & & & & & & $\mathrm{Y}$ & & & & $\mathrm{Y}$ & & $\mathrm{Y} / \mathrm{Y}$ & $Y$ & $\bar{Y}$ & & \\
\hline Pinus clausa & & $\mathrm{Y} / \mathrm{Y}$ & & & & & & & & & & & & & & & & & & & & & & & & & & & \\
\hline Pinus contorta & & $\mathrm{Y} / \mathrm{Y}$ & & & & & & & & & & & & & & & & & & & & & $\mathrm{Y}$ & & & & & & \\
\hline Pinus echinata & & $\mathrm{Y} / \mathrm{Y}$ & & & & & & & & & & & & & & & & & & & & & & & & & & & \\
\hline Pinus elliotii & & $\mathrm{Y} / \mathrm{Y}$ & & & $\mathrm{Y}$ & $\mathrm{Y}$ & & & & & & & $\mathrm{Y}$ & & & & & & $\mathrm{Y}$ & & & $\mathrm{Y}$ & & & & & & & \\
\hline Pinus mugo & & $\mathrm{Y} /-$ & & & & & & & & & & & & & & & & & & & & & $\mathrm{Y}$ & & & & & & \\
\hline Pinus palustrus & & $\mathrm{Y} / \mathrm{Y}$ & & & $\mathrm{Y}$ & $\mathrm{Y}$ & & & & & & & $\mathrm{Y}$ & & & & & & $\mathrm{Y}$ & & & & & & & & & & \\
\hline Pinus ponderosa & & $\mathrm{Y} / \mathrm{Y}$ & & & & & & & & & & & & & & & & & & & & & & & & & & & \\
\hline Pinus strobus & & $\mathrm{Y} / \mathrm{Y}$ & & & & & & & & & & & & & & & & & $\mathrm{Y}$ & & & & $\mathrm{Y}$ & & & & & & \\
\hline Pinus sylvestris & & $\mathrm{Y} / \mathrm{Y}$ & & & & & & & & & & & & & & & & & $\mathrm{Y}$ & & & & $\mathrm{Y}$ & & & & & & \\
\hline Pinus taeda & & $\mathrm{Y} / \mathrm{Y}$ & & & $\mathrm{Y}$ & $\mathrm{Y}$ & & & & & & & $\mathrm{Y}$ & & $\mathrm{Y}$ & & & & $\mathrm{Y}$ & & & & & & & $\mathrm{Y}$ & $\mathrm{Y}$ & & \\
\hline
\end{tabular}




\begin{tabular}{|c|c|c|c|c|c|c|c|c|c|c|c|c|c|c|c|c|c|c|c|c|c|c|c|c|c|c|c|c|c|c|}
\hline Scientific name & 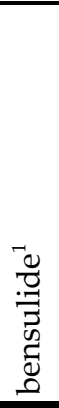 & 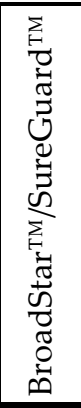 & 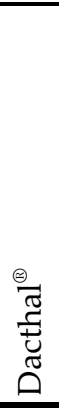 & 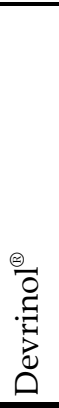 & 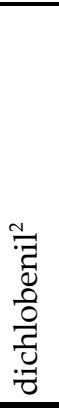 & 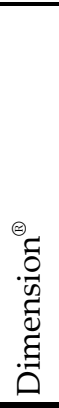 & 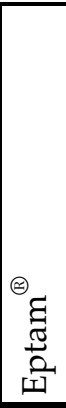 & 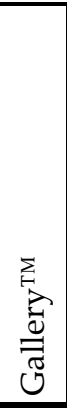 & $\begin{array}{l}\Theta_{0} \\
\infty \\
\widetilde{\Xi} \\
\Xi\end{array}$ & 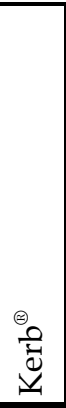 & 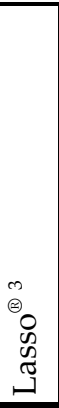 & तี & 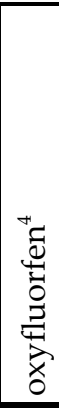 & 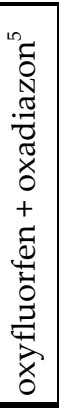 & 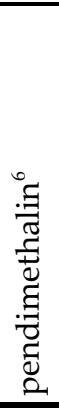 & 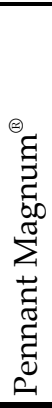 & 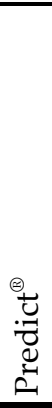 & 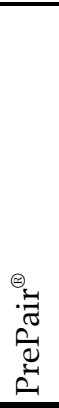 & 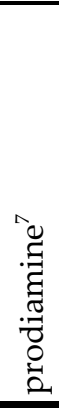 & 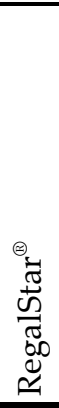 & 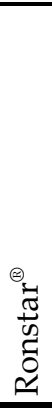 & 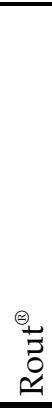 & $\begin{array}{l}0 \\
\text { d } \\
\text { d } \\
3 \\
0 \\
0 \\
\text { क }\end{array}$ & . & 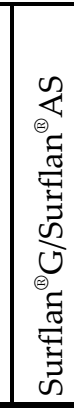 & 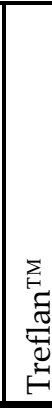 & & 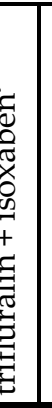 & 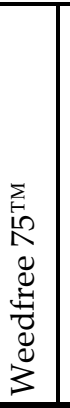 & $\stackrel{\oplus}{\vec{x}}$ \\
\hline Pinus virginiana & & $\mathrm{Y} / \mathrm{Y}$ & & & & & & & & & & & & & & & & & $\mathrm{Y}$ & & & & & & & & & & & \\
\hline Pinus spp. & & & $\mathrm{Y}$ & $\mathrm{Y}$ & $\mathrm{Y}$ & $\mathrm{Y}^{11}$ & & $Y^{11}$ & & $\mathrm{Y}$ & & $\mathrm{Y}$ & $\mathrm{Y}^{11}$ & $\mathrm{Y}$ & $\mathrm{Y}^{11}$ & $Y$ & & $\mathrm{Y}$ & $\mathrm{Y}^{11}$ & $\mathrm{Y}$ & $\mathrm{Y}$ & $\mathrm{Y}^{11}$ & & $\mathrm{Y}^{11}$ & $\mathrm{Y} / \mathrm{Y}^{1}$ & $\mathrm{Y}^{1}$ & & 11 & & $\bar{Y}$ \\
\hline Pittosporum tobira & & & & $\mathrm{Y}$ & $\mathrm{Y}$ & $\mathrm{Y}$ & & $\mathrm{Y}$ & & & & & & & & $\mathrm{Y}$ & & $\mathrm{Y}$ & $\mathrm{Y}$ & $\mathrm{Y}$ & $\mathrm{Y}$ & $\mathrm{Y}^{11}$ & $\bar{Y}$ & & $\bar{Y} / \mathrm{Y}^{1}$ & $Y^{1}$ & & 11 & & $\mathrm{Y}$ \\
\hline Pittosporum spp. & & $\mathrm{Y} /-$ & & $\mathrm{Y}$ & $\mathrm{Y}$ & & & & & & & $\mathrm{Y}$ & & $\mathrm{Y}$ & & & & & $Y^{11}$ & $Y^{11}$ & & $\mathrm{Y}^{11}$ & & & $\overline{Y / Y}$ & & & & & \\
\hline Platanus occidentalis & & $Y / Y^{11}$ & $\mathrm{Y}$ & & & $\mathrm{Y}$ & & $\mathrm{Y}$ & & $\mathrm{Y}$ & & & $\mathrm{Y}$ & & $\mathrm{Y}$ & & $\mathrm{Y}$ & & & & & & & & $\mathrm{Y} / \mathrm{Y}$ & Y & & $Y$ & & $Y$ \\
\hline Platanus racemosa & & $\mathrm{Y} / \mathrm{Y}$ & & & & & & & & & & & & & & & & & & & & & & & & & & & & \\
\hline $\begin{array}{l}\text { Platycladus orientalis } \\
\text { (synonym Thuja orientalis) }\end{array}$ & & $\mathrm{Y} / \mathrm{Y}$ & $\mathrm{Y}$ & & $\mathrm{Y}$ & $\mathrm{Y}$ & & $\mathrm{Y}$ & & $\mathrm{Y}$ & & $\mathrm{Y}$ & Y & $Y$ & & $\mathrm{Y}$ & & $\mathrm{Y}$ & & $\mathrm{Y}$ & $\mathrm{Y}$ & $\mathrm{Y}$ & $\mathrm{Y}$ & $\mathrm{Y}$ & $\mathrm{Y} / \mathrm{Y}$ & $\mathrm{Y}$ & & Y & & Y \\
\hline Paeonia cultivars & & & & & & & & & & & & & & & & & & & & $\mathrm{Y}$ & & $\mathrm{Y}$ & & & & & & & & \\
\hline Plumbago auriculata & & & & & & & & $\mathrm{Y}$ & & & & & & & & & & & & & & & & & $\mathrm{Y} / \mathrm{Y}$ & Y & & $Y$ & & $Y$ \\
\hline \multicolumn{31}{|l|}{$\begin{array}{l}\text { Podocarpus gracilior (see } \\
\text { Afrocarpus gracilior) }\end{array}$} \\
\hline Podocarpus macrophyllus & & & & & & & & & & & & & & & & & & & $\mathrm{Y}$ & & $\mathrm{Y}$ & & & & $\mathrm{Y} / \mathrm{Y}$ & $\bar{Y}$ & & $\bar{Y}$ & & $\mathrm{Y}$ \\
\hline Podocarpus spp. & & $\mathrm{Y} /-$ & $\mathrm{Y}$ & $\mathrm{Y}$ & $\mathrm{Y}$ & & & $\mathrm{Y}$ & & & & & & & & $\mathrm{Y}$ & & $\mathrm{Y}$ & & & & & $\mathrm{Y}$ & & $\mathrm{Y} / \mathrm{Y}$ & $Y$ & & $Y$ & & $Y$ \\
\hline Populus spp. & & $\mathrm{Y} / \mathrm{Y}$ & & & & & & & & & & & & & & & & & & & & & & & & & & & & \\
\hline Prunus caroliniana & & & & & & & & $\mathrm{Y}$ & & & & & & & & & & & & & & & & & $\mathrm{Y} / \mathrm{Y}$ & $\bar{Y}$ & & & & $Y$ \\
\hline Prunus laurocerasus & & & & & & & & $\mathrm{Y}$ & & & & & & & & & & & $\mathrm{Y}$ & $\mathrm{Y}$ & $\mathrm{Y}$ & & & & $\mathrm{Y} / \mathrm{Y}$ & & & & & $\mathrm{Y}$ \\
\hline Prunus spp. & & $\mathrm{Y} / \mathrm{Y}$ & & $\mathrm{Y}$ & & & & $\mathrm{Y}^{11}$ & & $\mathrm{Y}^{11}$ & & $\mathrm{Y}$ & $\bar{Y}$ & $\mathrm{Y}$ & $\mathrm{Y}^{11}$ & $\mathrm{Y}$ & & & $Y^{11}$ & & $\mathrm{Y}^{11}$ & $\mathrm{Y}^{11}$ & $\bar{Y}$ & & $\overline{Y / Y^{1}}$ & $Y^{1}$ & & 11 & & $\overline{Y^{11}}$ \\
\hline \multicolumn{31}{|l|}{$\begin{array}{l}\text { Psidium littorale var. } \\
\text { lucidum } \\
\text { (synonym P. cattleianum) }\end{array}$} \\
\hline Pyracantha coccinea & & & & & & & & & & & & & & & $\mathrm{Y}$ & & & & $\mathrm{Y}$ & $\mathrm{Y}$ & $\mathrm{Y}$ & & & & $\mathrm{Y} / \mathrm{Y}$ & & & & & $Y$ \\
\hline Pyracantha koidzumii & & & & & & $Y^{11}$ & & & & & & & & & & & & & $\mathrm{Y}$ & & & & & & $\mathrm{Y} / \mathrm{Y}$ & & & & & \\
\hline $\begin{array}{l}\text { Pyracantha. crenatoserrata } \\
\text { (synonym } P \text {. fortuneana) }\end{array}$ & & & & & & & & & & & & & & & Y & & & & Y & & & $Y$ & & & $\mathrm{Y} / \mathrm{Y}$ & & & 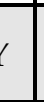 & & \\
\hline
\end{tabular}




\begin{tabular}{|c|c|c|c|c|c|c|c|c|c|c|c|c|c|c|c|c|c|c|c|c|c|c|c|c|c|c|c|c|c|}
\hline Scientific name & 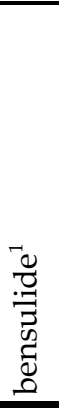 & 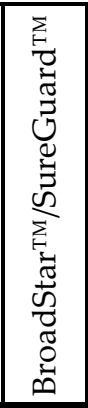 & 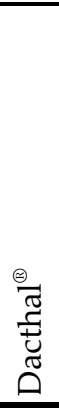 & 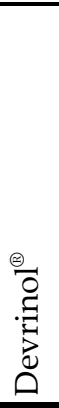 & 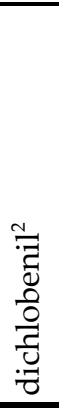 & 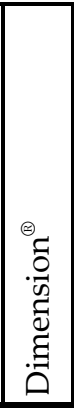 & 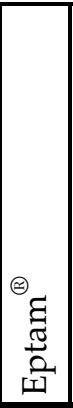 & 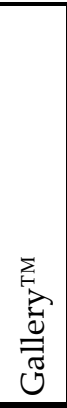 & $\begin{array}{l}\Theta_{0} \\
\infty \\
\Xi \\
\Xi\end{array}$ & 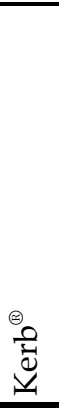 & 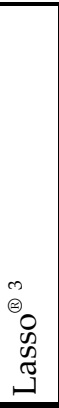 & $\begin{array}{l}\stackrel{\oplus}{\stackrel{7}{J}} \\
\stackrel{0}{0}\end{array}$ & 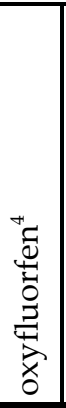 & 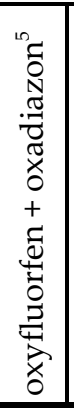 & 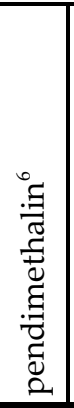 & 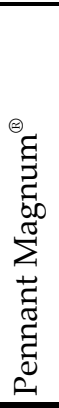 & 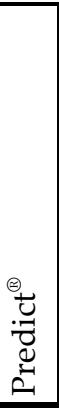 & 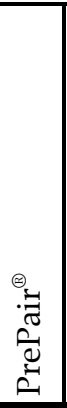 & 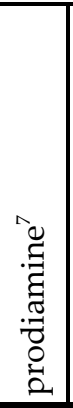 & 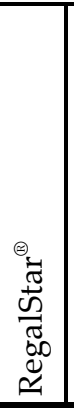 & 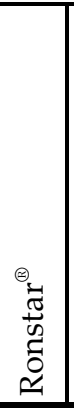 & $\begin{array}{l}\Theta_{1} \\
\vec{z} \\
\stackrel{0}{*}\end{array}$ & 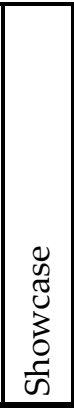 & 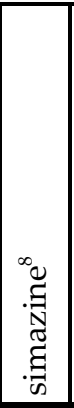 & 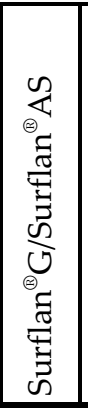 & 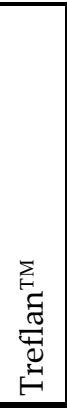 & 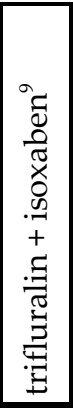 & 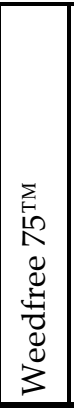 & 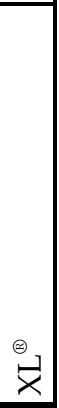 \\
\hline Pyracantha spp. & $\mathrm{Y}$ & $\mathrm{Y} /-$ & & $\mathrm{Y}$ & $\mathrm{Y}$ & $Y^{11}$ & & $Y^{11}$ & & $\mathrm{Y}$ & & $\mathrm{Y}$ & & $\mathrm{Y}$ & & $\mathrm{Y}$ & & $\mathrm{Y}$ & $\mathrm{Y}^{11}$ & & & & $Y^{11}$ & & & $\mathrm{Y}$ & & & \\
\hline Pyrus calleryana 'Bradford' & & & & & & & & & & $\mathrm{Y}$ & & & & & $\mathrm{Y}$ & $\mathrm{Y}$ & & & $\mathrm{Y}$ & & & & & & & & & & \\
\hline Pyrus spp. & & $\mathrm{Y}^{11} / \mathrm{Y}$ & & & & & & & & & & & & & & & & & & & & & & & & & & & \\
\hline Quercus ilicifolia & & $\mathrm{Y} /-$ & & & & & & & & & & & & & & & & & & & & & $\mathrm{Y}$ & & & & & & \\
\hline Quercus laurifolia & & & & & & $\mathrm{Y}$ & & & & & & & & & & & & & & & & & & & & & & & \\
\hline Quercus nigra & & & & & & & & & & & & & $\mathrm{Y}$ & & $\mathrm{Y}$ & & & & & & & & & & & & & & \\
\hline Quercus palustris & & $\mathrm{Y} /-$ & & $\mathrm{Y}$ & & $\mathrm{Y}$ & & $\mathrm{Y}$ & & & & & $\mathrm{Y}$ & & $\mathrm{Y}$ & & $\mathrm{Y}$ & & & & & & $\mathrm{Y}$ & & $-/ Y$ & $\mathrm{Y}$ & $\mathrm{Y}$ & & \\
\hline Quercus phellos & & $\mathrm{Y} /-$ & & & & $\mathrm{Y}$ & & $\mathrm{Y}$ & & & & & $\mathrm{Y}$ & & $\mathrm{Y}$ & & $\mathrm{Y}$ & & & & & & $\mathrm{Y}$ & & $-/ Y$ & $\mathrm{Y}$ & $\mathrm{Y}$ & & \\
\hline Quercus prinus & & & & & & & & & & $\mathrm{Y}$ & & $\mathrm{Y}$ & $\mathrm{Y}$ & $\mathrm{Y}$ & $\mathrm{Y}^{11}$ & $\mathrm{Y}$ & & & & $\mathrm{Y}$ & $\mathrm{Y}$ & & & & & $\mathrm{Y}^{11}$ & $\mathrm{Y}^{11}$ & & $\mathrm{Y}$ \\
\hline Quercus rubra & & $\mathrm{Y} /-$ & & & & $\mathrm{Y}$ & & $\mathrm{Y}$ & & & & & $\mathrm{Y}$ & & $\mathrm{Y}$ & & & & $\mathrm{Y}$ & & & & $\mathrm{Y}$ & $\mathrm{Y}$ & $-/ Y$ & $\mathrm{Y}$ & $\mathrm{Y}$ & & \\
\hline Quercus shumardii & & & & & & & & & & & & & & & & & & & $\mathrm{Y}$ & & & & & & & & & & \\
\hline Quercus virginiana & & $\mathrm{Y} /-$ & & & & $\mathrm{Y}$ & & $\mathrm{Y}$ & & & & & & & $\mathrm{Y}$ & & & & & & & & $Y$ & & & $\mathrm{Y}$ & $\mathrm{Y}$ & & \\
\hline Quercus spp. & & $-/ Y$ & $\mathrm{Y}$ & & $\mathrm{Y}$ & & & & & & & $\mathrm{Y}$ & & $\mathrm{Y}$ & & $\mathrm{Y}$ & & $\mathrm{Y}$ & & $\mathrm{Y}$ & $\mathrm{Y}$ & & & & $\mathrm{Y} / \mathrm{Y}$ & $\mathrm{Y}^{11}$ & $\mathrm{Y}^{11}$ & & \\
\hline Rhaphiolepis indica & & $\mathrm{Y} /-$ & & $\mathrm{Y}$ & & & & $Y^{11}$ & $\mathrm{Y}$ & & & $\mathrm{Y}$ & & $\mathrm{Y}$ & $\mathrm{Y}$ & $\mathrm{Y}$ & & $\mathrm{Y}$ & $\bar{Y}$ & $\mathrm{Y}$ & & $\mathrm{Y}^{11}$ & $\mathrm{Y}$ & & $\mathrm{Y} / \mathrm{Y}^{11}$ & $\mathrm{Y}$ & $\mathrm{Y}^{11}$ & & $Y^{11}$ \\
\hline Rhaphiolepis umbellata & & & & $\mathrm{Y}$ & & & & & & & & $\mathrm{Y}$ & & $\mathrm{Y}$ & & $\mathrm{Y}$ & & $\mathrm{Y}$ & $\bar{Y}$ & & $\mathrm{Y}^{11}$ & & & & & & & & \\
\hline Rhapis excelsa & & & & & & & & & & & & & & & & & & & & & $\mathrm{Y}$ & & & & & & & & \\
\hline Rhododendron spp. & $\mathrm{Y}$ & $\mathrm{Y} /-$ & $\mathrm{Y}$ & $\mathrm{Y}$ & $\mathrm{Y}^{11}$ & $\mathrm{Y}^{11}$ & $\mathrm{Y}$ & $Y^{11}$ & $\mathrm{Y}^{11}$ & $\mathrm{Y}$ & & $\mathrm{Y}^{11}$ & & $\mathrm{Y}^{11}$ & $\mathrm{Y}$ & $\mathrm{Y}$ & & $\mathrm{Y}^{11}$ & $\mathrm{Y}^{11}$ & $\mathrm{Y}^{11}$ & $\mathrm{Y}^{11}$ & $\mathrm{Y}^{11}$ & $\mathrm{Y}^{11}$ & $\mathrm{Y}$ & $\mathrm{Y} / \mathrm{Y}$ & $\mathrm{Y}$ & $\mathrm{Y}^{11}$ & & $\mathrm{Y}$ \\
\hline Rosa spp. & & $\mathrm{Y} /-$ & $\mathrm{Y}$ & $\mathrm{Y}$ & $\mathrm{Y}$ & & & $Y^{11}$ & & & & & & & $\mathrm{Y}$ & $\mathrm{Y}$ & & $\mathrm{Y}$ & $\mathrm{Y}^{11}$ & & $\mathrm{Y}$ & & & & $\mathrm{Y} / \mathrm{Y}$ & $\mathrm{Y}$ & $\mathrm{Y}^{11}$ & & $\mathrm{Y}$ \\
\hline Rosmarinus officinalis & & & & & & $\mathrm{Y}$ & & $\mathrm{Y}$ & & & & & & & $\mathrm{Y}$ & & & & & & & & & & $\mathrm{Y} / \mathrm{Y}$ & $\mathrm{Y}$ & $\mathrm{Y}$ & & $\mathrm{Y}$ \\
\hline Salix babylonica & & & & & & & & $\mathrm{Y}$ & & & & & & & $\mathrm{Y}$ & & & & & & & & & & $\mathrm{Y} / \mathrm{Y}$ & & $\mathrm{Y}$ & & $\mathrm{Y}$ \\
\hline Salix spp. & & & $\mathrm{Y}$ & & $\mathrm{Y}$ & & & & & $\mathrm{Y}$ & & & & & & $\mathrm{Y}$ & & & & & & & & & & $\mathrm{Y}$ & & & \\
\hline Spiraea cantoniensis & & $\mathrm{N} /-$ & $\mathrm{Y}$ & & $\mathrm{Y}$ & $\mathrm{Y}$ & & & & $\mathrm{Y}$ & & $\mathrm{Y}$ & & $\mathrm{Y}$ & & $\mathrm{Y}$ & & & & & & & & & & & & & \\
\hline Spiraea japonica & & $\mathrm{N} /-$ & $\mathrm{Y}$ & & $\mathrm{Y}$ & $\mathrm{Y}$ & & $\mathrm{Y}$ & & $\mathrm{Y}$ & & $\mathrm{Y}$ & & $\mathrm{Y}$ & $\mathrm{Y}$ & $\mathrm{Y}$ & & & $\mathrm{Y}$ & & & $\mathrm{Y}$ & $\mathrm{Y}$ & & & $\mathrm{Y}$ & $\mathrm{Y}$ & & \\
\hline Spiraea $\times$ vanhouttei & & $\mathrm{N} /-$ & $\mathrm{Y}$ & & $\mathrm{Y}$ & $\mathrm{Y}$ & & $\mathrm{Y}$ & & $\mathrm{Y}$ & & $\mathrm{Y}$ & & $\mathrm{Y}$ & $\mathrm{Y}$ & $\mathrm{Y}$ & & & & & & & & & $\mathrm{Y} / \mathrm{Y}$ & $\mathrm{Y}$ & $\mathrm{Y}$ & & $\mathrm{Y}$ \\
\hline
\end{tabular}




\begin{tabular}{|c|c|c|c|c|c|c|c|c|c|c|c|c|c|c|c|c|c|c|c|c|c|c|c|c|c|c|c|c|c|}
\hline Scientific name & 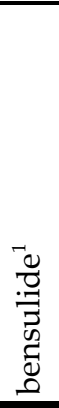 & 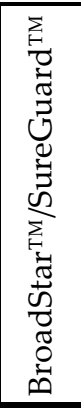 & 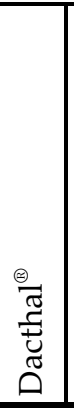 & 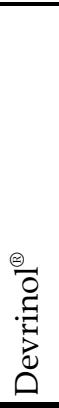 & 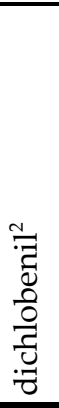 & 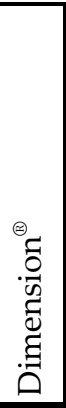 & 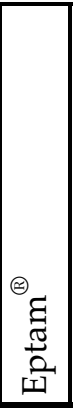 & 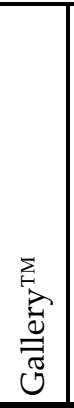 & $\begin{array}{l}\Theta_{0} \\
\stackrel{\infty}{\Xi} \\
\Xi \\
\Xi\end{array}$ & 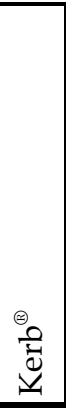 & 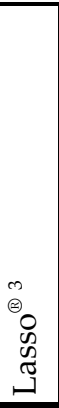 & 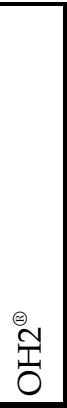 & 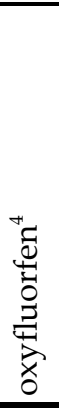 & 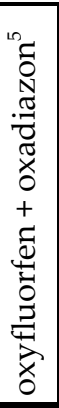 & 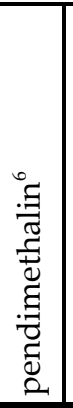 & 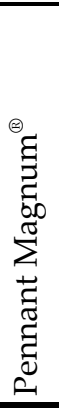 & 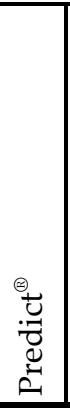 & 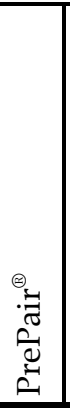 & 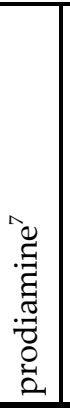 & 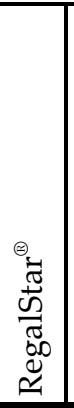 & $\begin{array}{c}\Theta_{1} \\
\stackrel{\tilde{J}}{0} \\
\stackrel{0}{0} \\
\tilde{0} \\
\simeq\end{array}$ & 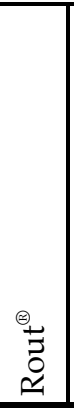 & 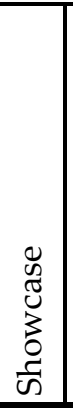 & 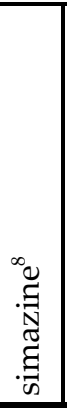 & 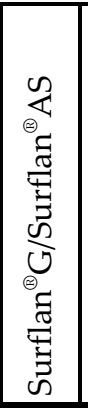 & 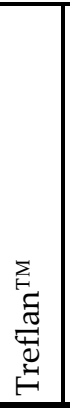 & 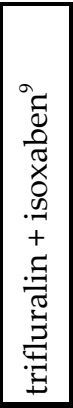 & 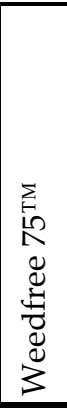 & 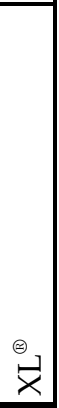 \\
\hline Swietenia mahogani & & & & & & & & $\mathrm{Y}$ & & & & & $\mathrm{Y}$ & & & & & & & & & & & & $\mathrm{Y} / \mathrm{Y}$ & $\mathrm{Y}$ & $\mathrm{Y}$ & & \\
\hline Syagrus romanzoffianum & & & & & & $\mathrm{Y}$ & & $\mathrm{Y}$ & & & & & & & & & & & & & & & & & $\mathrm{Y} / \mathrm{Y}$ & $\mathrm{Y}$ & & & $\mathrm{Y}$ \\
\hline Tabebuia caraiba & & & & & & & & $\mathrm{Y}$ & & & & & & & & & & & & & & & & & $\mathrm{Y} / \mathrm{Y}$ & $\mathrm{Y}$ & $\mathrm{Y}$ & & $\mathrm{Y}$ \\
\hline Taxodium distichum & & & $\mathrm{Y}$ & $\mathrm{Y}$ & & $\mathrm{Y}$ & & & & & & & & & $\mathrm{Y}$ & $\mathrm{Y}$ & $\mathrm{Y}$ & $\mathrm{Y}$ & & & $\mathrm{Y}$ & & & & & $\mathrm{Y}$ & & & \\
\hline Ternstroemia gymnanthera & & & & & & & & $\mathrm{Y}$ & & & & & & & & $\mathrm{Y}$ & & & & & & $\mathrm{Y}$ & $\mathrm{Y}$ & & & $\mathrm{Y}$ & $\mathrm{Y}$ & & \\
\hline Thuja occidentalis & & $\mathrm{Y} / \mathrm{Y}$ & $\mathrm{Y}$ & & $\mathrm{Y}$ & $\mathrm{Y}$ & & $\mathrm{Y}$ & & $\mathrm{Y}$ & & $\mathrm{Y}$ & $\mathrm{Y}$ & $\mathrm{Y}$ & $\mathrm{Y}$ & $\mathrm{Y}$ & & & $\mathrm{Y}$ & & & $\mathrm{Y}$ & $\mathrm{Y}$ & $\mathrm{Y}$ & $\mathrm{Y} / \mathrm{Y}^{11}$ & $\mathrm{Y}$ & $\mathrm{Y}$ & & $Y^{11}$ \\
\hline \multicolumn{30}{|l|}{$\begin{array}{l}\text { Thuja orientalis (see } \\
\text { Platycladus orientalis) }\end{array}$} \\
\hline Tibouchina urvilleana & & & & & & & & $\mathrm{Y}$ & & & & & & & & & & & & & & & & & & & & & \\
\hline Trqchycarpus fortunei & & & & & & $\mathrm{Y}$ & & & & & & & & & & & & & & & & & & & & & & & \\
\hline Ulmus parvifolia & & $\mathrm{Y} /-$ & $\mathrm{Y}$ & & & $\mathrm{Y}$ & & $\mathrm{Y}$ & & & & & & & & & & & & & & & & & & & & & \\
\hline Ulmus spp. & & & & & $\mathrm{Y}$ & & & & & $\mathrm{Y}$ & & & & & $\mathrm{Y}^{11}$ & & & & & & $\mathrm{Y}$ & & & & & & & & \\
\hline Veitchia merrillii & & & & & & & & $\mathrm{Y}$ & & & & & & & & & & $\mathrm{Y}$ & & & & & & & $\mathrm{Y} / \mathrm{Y}$ & $\mathrm{Y}$ & $\mathrm{Y}$ & & \\
\hline Viburnum japonicum & & & & & & & & $\mathrm{Y}$ & & & & & & & & & & & & $\mathrm{Y}$ & & & $\mathrm{Y}$ & & $\mathrm{Y} / \mathrm{Y}$ & & $Y$ & & $\mathrm{Y}$ \\
\hline Viburnum suspensum & & & $\mathrm{Y}$ & & & & & & & & & & & & $\mathrm{Y}$ & & & $\mathrm{Y}^{11}$ & & & & & & & $\mathrm{Y} / \mathrm{Y}$ & & & & $\mathrm{Y}$ \\
\hline Viburnum spp. & & $\mathrm{Y} /-$ & & $\mathrm{Y}$ & & $\mathrm{Y}^{11}$ & $\mathrm{Y}$ & $\mathrm{Y}^{11}$ & $\mathrm{~N}$ & $\mathrm{Y}$ & & $\mathrm{Y}$ & & $\mathrm{Y}$ & & $\mathrm{Y}$ & & $\mathrm{Y}^{11}$ & $\mathrm{Y}$ & & $\mathrm{Y}^{11}$ & $\mathrm{Y}^{11}$ & $\mathrm{Y}^{11}$ & & $\mathrm{Y} / \mathrm{Y}^{11}$ & $\mathrm{Y}^{11}$ & $\mathrm{Y}^{11}$ & & $Y^{11}$ \\
\hline Washingtonia robusta & & & & $\mathrm{Y}$ & & $\mathrm{Y}$ & & $\mathrm{Y}$ & & & & & & & & $\mathrm{Y}$ & & & & & & & & & $\mathrm{Y} / \mathrm{Y}$ & $\mathrm{Y}$ & $\mathrm{Y}$ & & $\mathrm{Y}$ \\
\hline Weigela florida & & $\mathrm{Y} /-$ & $\mathrm{Y}$ & & $\mathrm{Y}$ & & & $\mathrm{Y}$ & & & & $\mathrm{Y}$ & & $\mathrm{Y}$ & $\mathrm{Y}$ & $\mathrm{Y}$ & & & $Y$ & $\mathrm{Y}$ & & $\mathrm{Y}$ & & $\mathrm{Y}$ & $\mathrm{Y} / \mathrm{Y}$ & $\mathrm{Y}$ & $\mathrm{Y}$ & & $\mathrm{Y}$ \\
\hline Xylosma congestum & $\mathrm{Y}$ & & & $\mathrm{Y}$ & & $\mathrm{Y}$ & & $\mathrm{Y}$ & & & & $\mathrm{Y}$ & & $\mathrm{Y}$ & $\mathrm{Y}$ & & & $\mathrm{Y}$ & & $\mathrm{Y}$ & $\mathrm{Y}$ & $\mathrm{Y}$ & $\mathrm{Y}$ & & $\mathrm{Y} / \mathrm{Y}$ & $\mathrm{Y}$ & $\mathrm{Y}$ & & $\mathrm{Y}$ \\
\hline \multicolumn{30}{|c|}{ GROUNDCOVERS AND VINING PLANTS } \\
\hline Aquilegia spp. \& cultivars & & & $\mathrm{Y}$ & & & $\mathrm{Y}$ & & $\mathrm{Y}^{11}$ & & & & & & & $\mathrm{Y}^{11}$ & $\mathrm{Y}$ & & & $\mathrm{Y}$ & & & & & & & & & & \\
\hline Aspidistra elatior & & & & & & & & $\mathrm{Y}$ & & & & & & & & & & & & & & & & & & & & & \\
\hline Calluna vulgaris & & & & $\mathrm{Y}$ & & & & $\mathrm{Y}$ & & & & & & & & & & & $\mathrm{Y}$ & & $\mathrm{Y}$ & & & & & $\mathrm{Y}$ & $\mathrm{Y}$ & & \\
\hline Cortaderia selloana. & & & & & & $\mathrm{Y}$ & & $\mathrm{Y}$ & & & & $\mathrm{Y}$ & & & $\mathrm{Y}$ & $\mathrm{Y}$ & & & $\mathrm{Y}$ & $\mathrm{Y}$ & & & & & $\mathrm{Y} / \mathrm{Y}$ & $\mathrm{Y}$ & $\mathrm{Y}$ & & $\mathrm{Y}$ \\
\hline
\end{tabular}




\begin{tabular}{|c|c|c|c|c|c|c|c|c|c|c|c|c|c|c|c|c|c|c|c|c|c|c|c|c|c|c|c|c|c|}
\hline Scientific name & 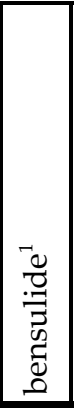 & 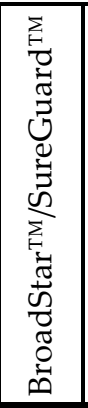 & 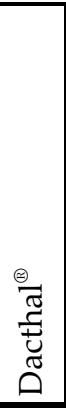 & 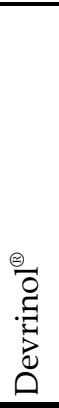 & 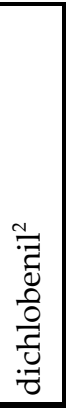 & 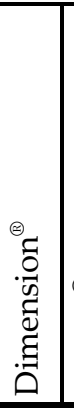 & 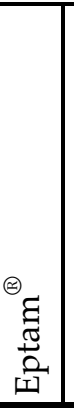 & 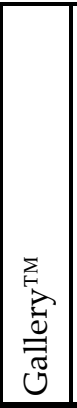 & $\begin{array}{l}\Theta_{0} \\
\infty \\
\Xi \\
\Xi\end{array}$ & 焉 & 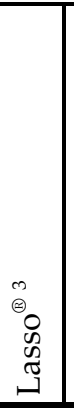 & 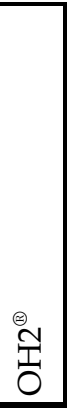 & 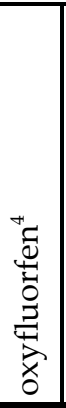 & 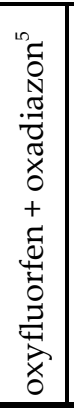 & 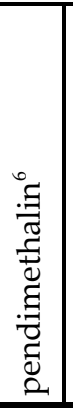 & 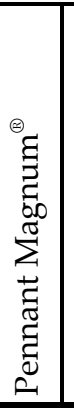 & 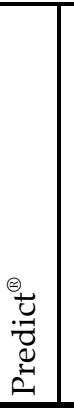 & 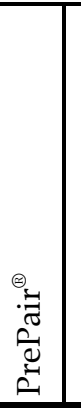 & 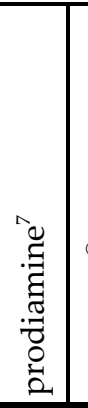 & 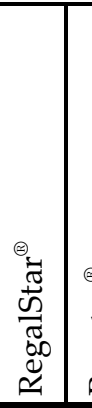 & 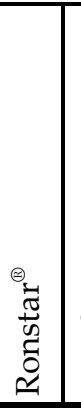 & 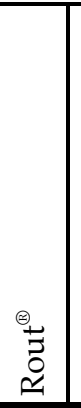 & \begin{tabular}{|c|} 
\\
$\tilde{z}$ \\
$\tilde{U}$ \\
3 \\
0 \\
ऊ \\
\end{tabular} & 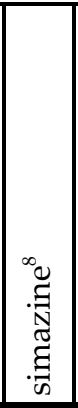 & 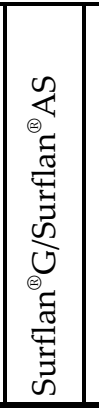 & 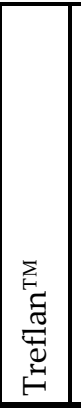 & 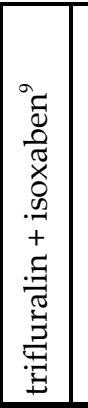 & 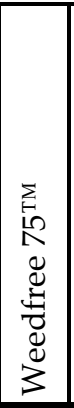 & \begin{tabular}{|l||} 
\\
$\stackrel{\ominus}{X}_{\times}$ \\
\end{tabular} \\
\hline $\begin{array}{l}\text { Dietes iridioides (synonym } \\
\text { Dietes vegeta) }\end{array}$ & & & & & & & & Y & & & & & & & & & & & & & & & & & $-/ Y$ & & & & \\
\hline Gelsemium sempervirens & & & & & & $\mathrm{Y}$ & & $\mathrm{Y}$ & & & & & & & & $\mathrm{Y}$ & & & & & & $\mathrm{Y}$ & $\mathrm{Y}$ & & & $\mathrm{Y}$ & $\mathrm{Y}$ & & \\
\hline Hedera canariensis & $Y$ & & & $\mathrm{Y}$ & & & & $Y$ & & & & & & & & & & $\mathrm{Y}$ & & & $\mathrm{Y}$ & & & & $\mathrm{Y} / \mathrm{Y}$ & $\mathrm{Y}$ & $\mathrm{Y}$ & & $\mathrm{Y}$ \\
\hline Hedera helix & $\mathrm{Y}$ & $\mathrm{Y} /-$ & $\mathrm{Y}$ & $\mathrm{Y}$ & $\mathrm{Y}$ & $\mathrm{Y}$ & & $Y$ & & & & & & & $\mathrm{Y}$ & $\mathrm{Y}$ & & $\mathrm{Y}$ & $\mathrm{Y}$ & & $\mathrm{Y}$ & & & & $\mathrm{Y} / \mathrm{Y}$ & $\mathrm{Y}$ & $\mathrm{Y}$ & & $\mathrm{Y}$ \\
\hline Hemerocallis spp. & & & & & & & & $\mathrm{Y}$ & & & & & & & $\mathrm{Y}$ & $\mathrm{Y}$ & & & $Y^{11}$ & & & & & & $\mathrm{Y} / \mathrm{Y}$ & $\mathrm{Y}$ & $\mathrm{Y}$ & & $\mathrm{Y}$ \\
\hline Hypericum spp. & $\mathrm{Y}$ & & & $\mathrm{Y}$ & & & $\mathrm{Y}$ & Y & & & & $\mathrm{Y}$ & & $\mathrm{Y}$ & $\mathrm{Y}$ & $\mathrm{Y}$ & & & & & $\mathrm{Y}$ & Y & & & $\mathrm{Y} / \mathrm{Y}$ & Y & Y & & $\mathrm{Y}$ \\
\hline Jasminum spp. & & & & & & & & $\mathrm{Y}^{11}$ & & & & $\mathrm{Y}$ & & $\mathrm{Y}$ & & $\mathrm{Y}$ & & & & & $\mathrm{Y}^{11}$ & & & & & & & & \\
\hline Jasminum mesnyi & & & & & & & & & & & & & & & $\mathrm{Y}$ & & & $\mathrm{Y}$ & & & $\mathrm{Y}$ & & & & & & & & \\
\hline Jasminum nitidum & & & & & & & & $\mathrm{Y}$ & & & & & & & & & & & & & & $\mathrm{Y}$ & & & & $\mathrm{Y}$ & $\mathrm{Y}$ & & \\
\hline Jasminum nudiflorum & & & & & & & & & & & & & & & & & & & $\bar{Y}$ & $\mathrm{Y}$ & & & & & & & & & \\
\hline Lantana camara & & & $\mathrm{Y}$ & $\mathrm{Y}$ & & & & $\mathrm{Y}$ & & & & & & & & & & & & & & & & & & $\mathrm{Y}$ & $\mathrm{Y}$ & & \\
\hline $\begin{array}{l}\text { Lantana sellowiana or } L \text {. } \\
\text { montevidensis }\end{array}$ & & & $\mathrm{Y}$ & $\mathrm{Y}$ & & & & Y & & & & & & & & Y & & Y & Y & & Y & Y & & & & Y & Y & & \\
\hline Lantana spp. & & $\mathrm{Y} /-$ & & & & & & & & & & & & & & & & & & & & & & & & & & & \\
\hline Liriope muscari & & & & $\mathrm{Y}$ & & $\mathrm{Y}$ & & $\mathrm{Y}^{11}$ & $\mathrm{Y}$ & & & & & & $\mathrm{Y}$ & $\mathrm{Y}$ & & & $Y^{11}$ & & & & & $\mathrm{Y}^{11}$ & $Y / Y^{11}$ & $\mathrm{Y}$ & $\mathrm{Y}$ & & $Y^{11}$ \\
\hline Liriope spicata & & & & $\mathrm{Y}$ & & $\mathrm{Y}$ & & $\mathrm{Y}$ & & & & & & & $\mathrm{Y}$ & $\mathrm{Y}$ & & & $\mathrm{Y}$ & & & & & & & $\mathrm{Y}$ & $\mathrm{Y}$ & & \\
\hline Lonicera japonica & & & & & & $\mathrm{Y}$ & & $\mathrm{Y}$ & & & & & & & & & & & & & & & & & $\mathrm{Y} / \mathrm{Y}$ & & & & $\mathrm{Y}$ \\
\hline Lonicera spp. & & $\mathrm{Y} /-$ & $\mathrm{Y}$ & & $\mathrm{Y}$ & & & $\mathrm{Y}$ & & & & $\mathrm{Y}$ & & $\mathrm{Y}$ & & $\mathrm{Y}$ & & & $\mathrm{Y}^{11}$ & & $\mathrm{Y}$ & $Y^{11}$ & $\mathrm{Y}^{11}$ & & $\mathrm{Y} / \mathrm{Y}^{11}$ & $Y^{11}$ & $\mathrm{Y}^{11}$ & & $Y^{11}$ \\
\hline Ophiopogon japonicus & & & & & & $\mathrm{Y}$ & & $\mathrm{Y}$ & $\mathrm{Y}$ & & & & & & $\mathrm{Y}$ & $\mathrm{Y}$ & & & $\mathrm{Y}$ & & & & & & $\mathrm{Y} / \mathrm{Y}$ & $\mathrm{Y}$ & $\mathrm{Y}$ & & $\mathrm{Y}$ \\
\hline Pachysandra terminalis & $\mathrm{Y}$ & $\mathrm{Y} /-$ & $\mathrm{Y}$ & $\mathrm{Y}$ & $\mathrm{Y}$ & $\mathrm{Y}$ & & $\mathrm{Y}$ & $\mathrm{Y}$ & & & $\mathrm{Y}$ & & $\mathrm{Y}$ & $\mathrm{Y}$ & $\mathrm{Y}$ & & & & \begin{tabular}{l|l}
$\mathrm{Y}$ & $\mathrm{Y}$ \\
\end{tabular} & $\mathrm{Y}$ & & & & $-/ Y$ & $\mathrm{Y}$ & $\mathrm{Y}$ & & $\mathrm{Y}$ \\
\hline Pennisetum spp. & & & & & & $\mathrm{Y}^{11}$ & & $Y^{11}$ & & & & & & & $Y^{11}$ & & & & $Y^{11}$ & & & & & & $-/ Y^{11}$ & $\mathrm{Y}^{11}$ & $\mathrm{Y}^{11}$ & & \\
\hline Rumohra adiantiformis & & & & & & & & & & & & & & & $\mathrm{Y}$ & $\mathrm{Y}$ & & & $\mathrm{Y}$ & & & & & & & & & & \\
\hline Ruscus hypophyllum & & & & & & & & $\mathrm{Y}$ & & & & & & & & & & & & & & & & & & & & & \\
\hline
\end{tabular}

\section{Preemergence Herbicides for Use in Ornamentals}




\begin{tabular}{|c|c|c|c|c|c|c|c|c|c|c|c|c|c|c|c|c|c|c|c|c|c|c|c|c|c|c|c|c|c|}
\hline Scientific name & 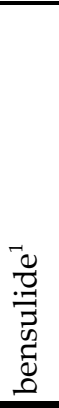 & 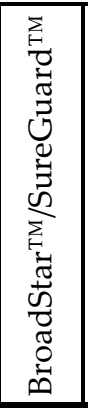 & 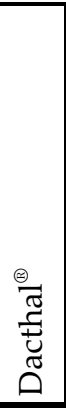 & 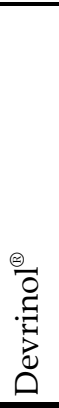 & 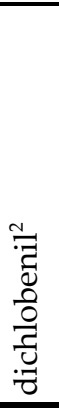 & 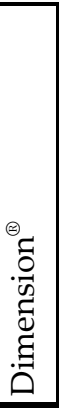 & 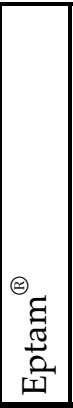 & 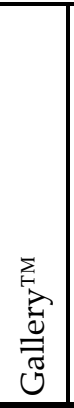 & 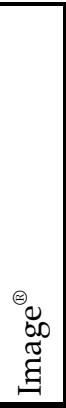 & 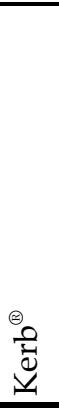 & 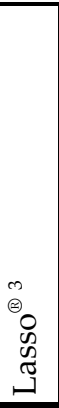 & ֻै & 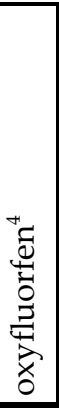 & 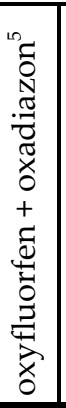 & 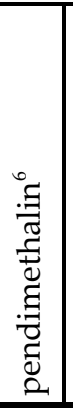 & 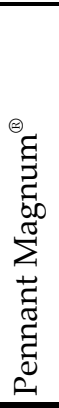 & 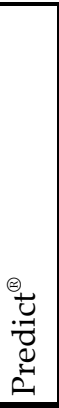 & 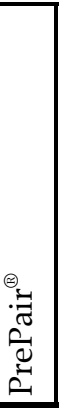 & 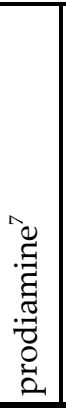 & 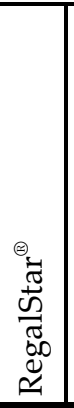 & 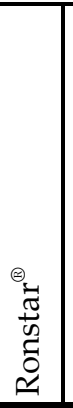 & 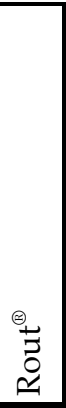 & $\begin{array}{l}\tilde{y} \\
\tilde{J} \\
3 \\
0 \\
\text { ஸे }\end{array}$ & 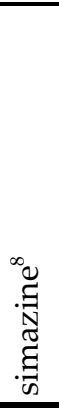 & 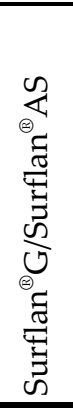 & 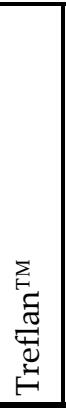 & 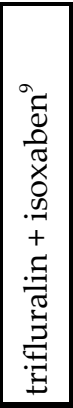 & 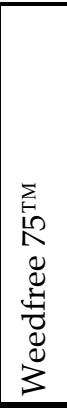 & $\stackrel{\Theta}{P}_{\vec{X}}$ \\
\hline $\begin{array}{l}\text { Trachelospermum } \\
\text { jasminoides }\end{array}$ & $\mathrm{Y}$ & & & $\mathrm{Y}$ & & & & & & & & & & & & & & Y & & & $\mathrm{Y}$ & $\mathrm{Y}$ & & & $\mathrm{Y} / \mathrm{Y}$ & & & & $\mathrm{Y}$ \\
\hline Trachelospermum asiaticum & $\mathrm{Y}$ & & & & & $\mathrm{Y}$ & & $\mathrm{Y}$ & $\mathrm{Y}$ & & & & & & $\mathrm{Y}^{11}$ & & & & $\mathrm{Y}$ & & & $\mathrm{Y}^{11}$ & & & & $\mathrm{Y}$ & $\mathrm{Y}$ & & \\
\hline Tulbaghia violacea & & & & & & & & $\mathrm{Y}$ & & & & & & & & & & & & & & & & & $-/ Y$ & & $\mathrm{Y}$ & & \\
\hline Wedelia trilobata & & & & & & & & & & & & & & & & & & & & & $\mathrm{Y}$ & & & & & & & & \\
\hline Wisteria sinensis & & & & & & & & & & & & & & & $\mathrm{Y}$ & $\mathrm{Y}$ & & & & & $\mathrm{Y}$ & & & & & & & & \\
\hline \multicolumn{30}{|c|}{ ANNUALS AND PERENNIALS } \\
\hline Achillea spp. & & & $\mathrm{Y}$ & & & $\mathrm{Y}$ & & & & & & & & & $\mathrm{Y}$ & $\mathrm{Y}$ & & & $\mathrm{Y}$ & & & & & & $\mathrm{Y} / \mathrm{Y}$ & $\mathrm{Y}$ & $\mathrm{Y}^{11}$ & & $\mathrm{Y}$ \\
\hline Agapanthus africanus & & & & $\mathrm{Y}$ & & $Y^{11}$ & & $\mathrm{Y}$ & & & & & & & & $\mathrm{Y}$ & & $\mathrm{Y}$ & $\mathrm{Y}$ & & $\mathrm{Y}$ & & & & $\mathrm{Y} / \mathrm{Y}$ & $\mathrm{Y}$ & $\mathrm{Y}$ & & $\mathrm{Y}$ \\
\hline Agapanthus orientalis & & & & & & & & & & & & & & & & & & & $\mathrm{Y}$ & & & & & & & & & & \\
\hline Ageratum spp. & & & $\mathrm{Y}$ & $\mathrm{Y}$ & & & & & & & & & & & $\mathrm{Y}$ & $\mathrm{Y}$ & & & & & & & & & & $\mathrm{Y}^{11}$ & & & \\
\hline Ajuga reptans & $\mathrm{Y}$ & & & $\mathrm{Y}$ & & $\mathrm{Y}$ & & $\mathrm{N}$ & & & & & & & $\mathrm{Y}$ & $\mathrm{Y}$ & & $\mathrm{Y}$ & & & $\mathrm{Y}$ & & & & $\mathrm{Y} / \mathrm{Y}$ & & $\mathrm{N}$ & & $\mathrm{Y}$ \\
\hline Allamanda cathartica & & & & & & & & & & & & & & & & & & & & & $\mathrm{Y}$ & & & & & & & & \\
\hline Allium spp. & & & & & & & & & & & & & & & & $\mathrm{Y}$ & & & $\mathrm{Y}^{11}$ & & & & & & & & & & \\
\hline \multicolumn{30}{|l|}{ Alstroemeria cultivars } \\
\hline Alyssum spp. & $\mathrm{Y}$ & & $\mathrm{Y}$ & & & & & & & & & & & & $\mathrm{Y}$ & $\mathrm{Y}$ & & & & & & & & & & $\mathrm{Y}$ & & & \\
\hline Amaranthus cultivars & & $\mathrm{N} /-$ & & & & $\mathrm{N}$ & & & & & & & & & & & & & & & & & & & & & & & \\
\hline \multicolumn{30}{|l|}{ Ammi majus } \\
\hline Anemone coronaria & & & & & & & & & & & & & & & $\mathrm{Y}$ & & & & $\mathrm{Y}^{11}$ & & & & & & & & & & \\
\hline Anthurium andraeanum & & & & & & & & & & & & & & & & & & & & & & $\mathrm{Y}$ & & & & & & & \\
\hline Antirrhinum majus & & & $\mathrm{Y}$ & & & $\mathrm{Y}$ & & & & & & & & & $\mathrm{Y}$ & $\mathrm{Y}$ & & & & & & & & & $\mathrm{Y} / \mathrm{Y}$ & $\mathrm{Y}$ & & & $\mathrm{Y}$ \\
\hline Aquilegia spp. \& cultivars & & & $\mathrm{Y}$ & & & $\mathrm{Y}$ & & $\mathrm{Y}^{11}$ & & & & & & & $\mathrm{Y}^{11}$ & $\mathrm{Y}$ & & & $\mathrm{Y}$ & & & & & & & & & & \\
\hline Ascelepias tuberosa & & & & & & & & & & & & & & & $\mathrm{Y}$ & $\mathrm{Y}$ & & & & & & & & & & & & & \\
\hline Asparagus spp. & & & & $\mathrm{Y}$ & & & & $\mathrm{Y}^{11}$ & & & & & & & $\mathrm{Y}$ & & & & & & & & & & $-/ Y$ & $\mathrm{Y}^{11}$ & $\mathrm{Y}^{11}$ & & \\
\hline
\end{tabular}




\begin{tabular}{|c|c|c|c|c|c|c|c|c|c|c|c|c|c|c|c|c|c|c|c|c|c|c|c|c|c|c|c|c|c|}
\hline Scientific name & 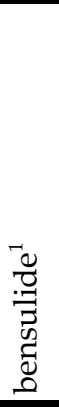 & 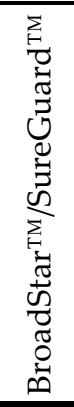 & 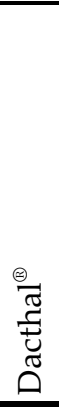 & 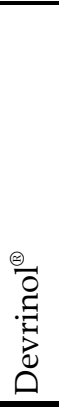 & 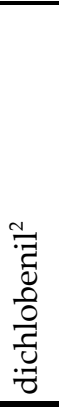 & 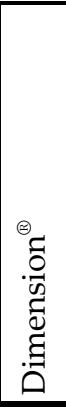 & 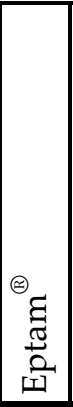 & 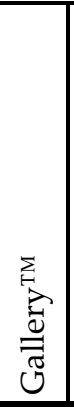 & $\begin{array}{l}\Theta_{0} \\
\stackrel{\infty}{\infty} \\
\Xi \\
\Xi\end{array}$ & 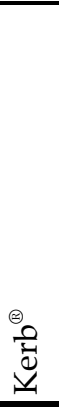 & 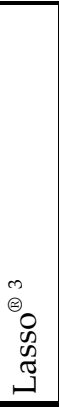 & $\stackrel{\oplus}{\stackrel{0}{1}}$ & 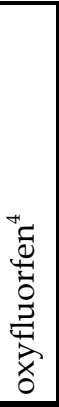 & 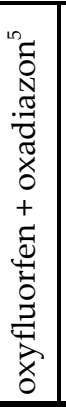 & 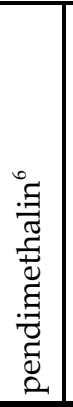 & 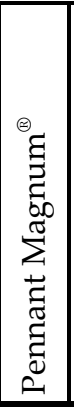 & 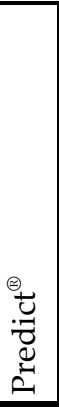 & 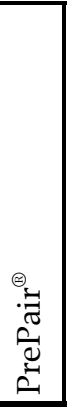 & 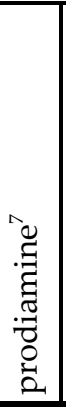 & 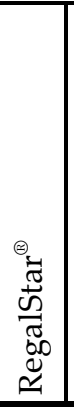 & 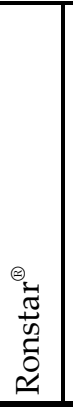 & $\begin{array}{l}\Theta_{1} \\
\vec{Z} \\
\stackrel{0}{a} \\
\end{array}$ & $\begin{array}{l}0 \\
0 \\
\tilde{J} \\
3 \\
0 \\
0 \\
\omega\end{array}$ & 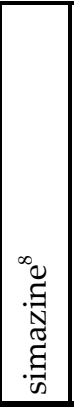 & 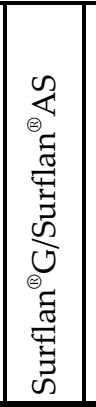 & 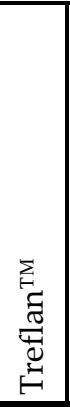 & 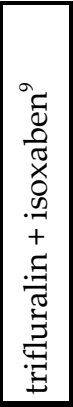 & 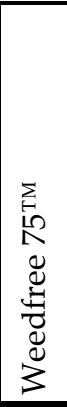 & $\stackrel{\oplus}{\times}$ \\
\hline Asparagus virgatus & & & & & & $\mathrm{Y}$ & & $\mathrm{Y}$ & & & & & & & $\mathrm{Y}$ & & & & & & & & & & & & $\mathrm{Y}$ & & \\
\hline Aspidistra elatior & & & & & & & & & & & & & & & $\mathrm{Y}$ & & & & & & & & & & & & & & \\
\hline Aster spp. & $\mathrm{Y}$ & & $\mathrm{Y}$ & $\mathrm{Y}$ & & & & $\mathrm{Y}^{11}$ & & & & & & & $\mathrm{Y}^{11}$ & $\mathrm{Y}$ & & & $\mathrm{Y}$ & & & & & & $-/ Y$ & $\mathrm{Y}$ & $\mathrm{Y}^{11}$ & & \\
\hline Astilbe cultivars & & & & & & & & & & & & & & & & & & & & & & & & & $\mathrm{Y} / \mathrm{Y}^{11}$ & $\mathrm{Y}^{11}$ & $\mathrm{Y}^{11}$ & & $\mathrm{Y}^{11}$ \\
\hline Athyrium nipponicum & & & & & & & & & & & & & & & & & & & & & & & & & $-/ Y$ & $\mathrm{Y}$ & & & \\
\hline Banksia menziesii & & & & & & & & & & & & & & & & & & & & & & $\mathrm{Y}$ & & & & & & & \\
\hline Begonia spp. & & & & & & $\mathrm{Y}$ & & & & & & & & & $\mathrm{Y}$ & & & & $\mathrm{Y}$ & & & & & & $\mathrm{N} / \mathrm{N}$ & $\mathrm{Y}$ & & & $\mathrm{N}$ \\
\hline \multicolumn{30}{|l|}{ Bouvardia hybrids } \\
\hline \multicolumn{30}{|l|}{$\begin{array}{l}\text { Bracteantha bracteata (see } \\
\text { Helichrysum bracteatum) }\end{array}$} \\
\hline Buddleia davidii & & $\mathrm{Y} /-$ & & & & & & & & & & & & & & & & & $\mathrm{Y}$ & & $\mathrm{Y}$ & & & & & & & & \\
\hline bulbs, ornamental & & & & & & & & $\mathrm{Y}$ & & & & & & & $\mathrm{Y}^{11}$ & $\mathrm{Y}^{11}$ & & & $\mathrm{Y}^{11}$ & & & & & & $\mathrm{Y} / \mathrm{Y}$ & $\mathrm{Y}^{11}$ & $\mathrm{Y}^{11}$ & & $\mathrm{Y}$ \\
\hline Caladium bicolor & & & & & & & & & & & & & & & $\mathrm{Y}$ & & & & & & & & & & $\mathrm{Y} / \mathrm{Y}$ & & & & $\mathrm{Y}$ \\
\hline Calendula officinalis & $\mathrm{Y}$ & & & & & & & & & & & & & & & & & & & & & & & & & $\mathrm{Y}$ & & & \\
\hline Campanula spp. & $\mathrm{Y}$ & & $\mathrm{Y}$ & & & & & & & & & & & & $\mathrm{Y}$ & $\mathrm{Y}^{11}$ & & & $\mathrm{Y}^{11}$ & & & & & & $\mathrm{Y} / \mathrm{Y}$ & $\mathrm{Y}$ & & & $\mathrm{Y}^{11}$ \\
\hline \multicolumn{30}{|l|}{ Carthamus tinctorius } \\
\hline \multicolumn{30}{|l|}{ Caryopteris incana } \\
\hline Catharanthus roseus & $\mathrm{Y}$ & & & & & & & & & & & & & & $\mathrm{Y}$ & & & & & & & & & & & & & & \\
\hline \multicolumn{30}{|l|}{ Cattleya cultivars } \\
\hline Celosia cristata & & & & & & $\mathrm{Y}$ & & & & & & & & & $\mathrm{Y}$ & & & & & & & & & & & & & & \\
\hline Chrysanthemum spp. & & & $\mathrm{Y}$ & $\mathrm{Y}$ & & & & $\mathrm{Y}$ & & & & & & & $\mathrm{Y}^{11}$ & $\mathrm{Y}$ & & & $\mathrm{Y}^{11}$ & & $\mathrm{Y}$ & & & & $\mathrm{Y} / \mathrm{Y}^{11}$ & $\mathrm{Y}$ & $\mathrm{Y}$ & & $Y^{11}$ \\
\hline Chrysanthemum superbum & $\mathrm{Y}$ & & & $\mathrm{Y}$ & & & & $\mathrm{Y}$ & & & & & & & $\mathrm{Y}$ & $\mathrm{Y}$ & & & & & $\mathrm{Y}$ & & & & & $\mathrm{Y}$ & $\mathrm{Y}$ & & $\mathrm{Y}$ \\
\hline \multicolumn{30}{|l|}{ Cirsium japonicum } \\
\hline Coleus spp. & & & $\mathrm{Y}$ & & & $\mathrm{Y}^{11}$ & & & & & & & & & & & & & & & & & & & $\mathrm{~N} / \mathrm{N}$ & & & & $\mathrm{N}$ \\
\hline
\end{tabular}




\begin{tabular}{|c|c|c|c|c|c|c|c|c|c|c|c|c|c|c|c|c|c|c|c|c|c|c|c|c|c|c|c|c|c|}
\hline Scientific name & 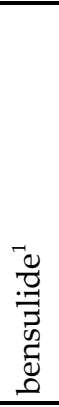 & 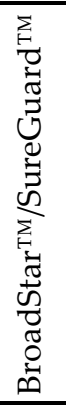 & 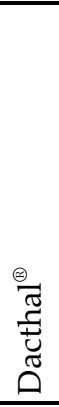 & 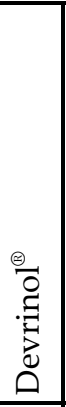 & 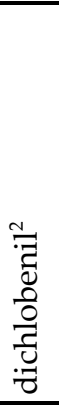 & 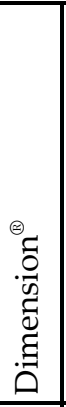 & ${ }^{\circledR} \approx$ & 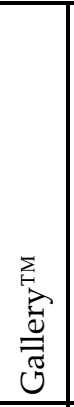 & $\begin{array}{l}\Theta_{0} \\
\infty \\
\Xi \\
\Xi\end{array}$ & 节 & $\begin{array}{l}0 \\
\Theta_{0} \\
\vdots \\
\tilde{O} \\
\Xi \\
\end{array}$ & 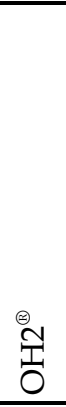 & 泀 & 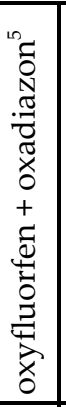 & 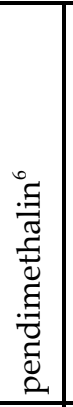 & 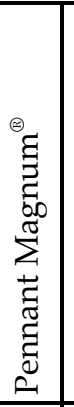 & 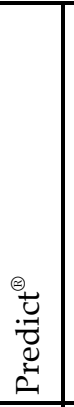 & 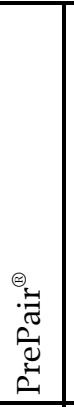 & 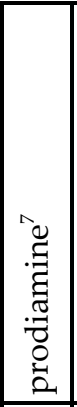 & 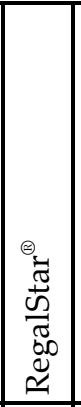 & 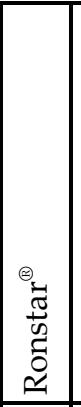 & 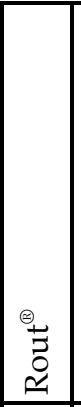 & 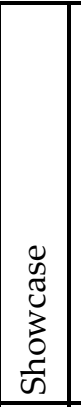 & 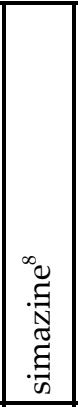 & 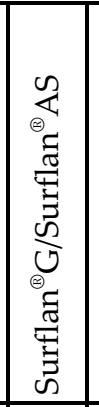 & 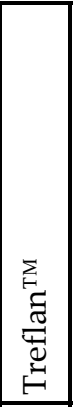 & 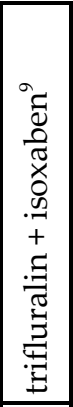 & 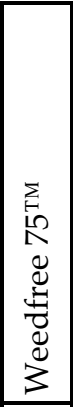 & $\stackrel{\oplus}{\stackrel{\oplus}{\times}}$ \\
\hline Coreopsis spp. & & & $\mathrm{Y}$ & & & $\mathrm{Y}$ & & $\mathrm{Y}$ & & & & & & & $Y^{11}$ & $\mathrm{Y}$ & & & $\bar{Y}$ & & $\bar{Y}$ & & & & $\mathrm{Y} / \mathrm{Y}^{11}$ & $\bar{Y}$ & $Y^{11}$ & & $Y^{11}$ \\
\hline Cosmos spp. & & & Y & & & & & & & & & & & & & & & & & & & & & & & Y & & & \\
\hline Costus spp. & & & & & & & & & & & & & & & & & & & & & & & & & & & & & \\
\hline Cuphea hyssopifolia & & & $\mathrm{Y}$ & & & & & $\mathrm{Y}$ & & & & & & & & & & & & & & & & & $-/ Y$ & & $\mathrm{Y}$ & & \\
\hline Cyathea australis & & & & & & & & & & & & & & & & & & & & & & & & & & & & & \\
\hline Cyclamen persicum & & & & & & & & & & & & & & & & & & & & & & & & & & & & & \\
\hline Cymbidium cultivars & & & & & & & & & & & & & & & & & & & & & & & & & & & & & \\
\hline Dahlia spp. & $\mathrm{Y}$ & & $\mathrm{Y}$ & $\mathrm{Y}$ & & & & & & & & & & & $\mathrm{Y}$ & & & & & & $\bar{Y}$ & & & & & $\bar{Y}$ & & & \\
\hline Delphinium spp. & & & $\mathrm{Y}$ & & & $\mathrm{Y}$ & & & & & & & & & & $\mathrm{Y}$ & & & $\mathrm{Y}$ & & & & & & & & & & \\
\hline $\begin{array}{l}\text { Dendranthema } \times \\
\text { grandiflorum (synonym } \\
\text { Chrysanthemum moriforium) }\end{array}$ & & & Y & $\mathrm{Y}$ & & & & $\mathrm{Y}$ & & & & & & & Y & Y & & & & & Y & & & & $\mathrm{Y} / \mathrm{Y}^{11}$ & Y & Y & & $\mathrm{Y}$ \\
\hline Dendrobium cultivars & & & & & & & & & & & & & & & & & & & & & & & & & & & & & \\
\hline Dianthus barbatus & & & $\mathrm{N}$ & & & & & $\mathrm{Y}^{11}$ & & & & & & & $\mathrm{Y}$ & $\mathrm{Y}$ & & & $\mathrm{Y}^{11}$ & & & & & & $\mathrm{Y} / \mathrm{Y}^{11}$ & $\bar{Y}$ & & & $\mathrm{Y}$ \\
\hline Dianthus caryophyllus & & & $\mathrm{N}$ & & & & & & & & & & 11 & & & $\mathrm{Y}^{11}$ & & & & & $\mathrm{Y}$ & & & & & $\bar{Y}$ & & & \\
\hline Dianthus plumarius & & & $\mathrm{N}$ & & & $\mathrm{Y}$ & & $\mathrm{Y}$ & & & & & & & $\mathrm{Y}^{11}$ & & & & $\mathrm{Y}^{11}$ & & & & & & & $\mathrm{Y}$ & & & \\
\hline Digitalis spp. & & & $\mathrm{Y}$ & & & & & & & & & & & & $\mathrm{Y}$ & & & & & & & & & & & $\mathrm{Y}^{11}$ & & & \\
\hline Dracaena spp. & & & & & & & & & & & & & & & & & & & & & & $\mathrm{Y}^{11}$ & & & & & & & \\
\hline Echinacea purpurea & & & & & & & & $\mathrm{N}$ & & & & & & & $\mathrm{Y}$ & & & & $\mathrm{Y}$ & & & & & & $\mathrm{Y} / \mathrm{Y}$ & $\mathrm{Y}$ & $\mathrm{Y}$ & & $\mathrm{Y}$ \\
\hline Erigeron cultivars & & & & & & & & & & & & & & & & & & & & & & & & & & & & & \\
\hline Eustoma grandiflorum & & & & & & & & & & & & & & & & & & & & & & & & & & & & & \\
\hline Freesia spp. & $\mathrm{Y}$ & & & & & & & & & & & & & & & & & & & & & & & & & & & & \\
\hline Gaillardia spp. & & & $\mathrm{Y}$ & & & & & & & & & & & & $\mathrm{Y}$ & $\mathrm{Y}$ & & & $\mathrm{Y}$ & & & & & & & $\bar{Y}$ & $\mathrm{Y}^{11}$ & & \\
\hline Gerbera jamesonii & & & & & & & & & & & & & & & & & & & & & & & & & & & & & \\
\hline
\end{tabular}




\begin{tabular}{|c|c|c|c|c|c|c|c|c|c|c|c|c|c|c|c|c|c|c|c|c|c|c|c|c|c|c|c|c|c|}
\hline Scientific name & 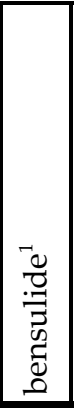 & 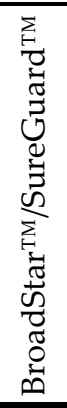 & 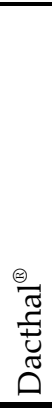 & 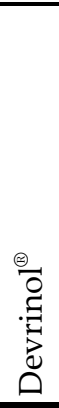 & 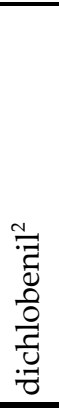 & 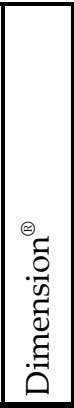 & 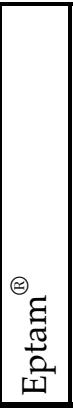 & 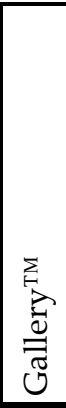 & $\begin{array}{l}\Theta_{0} \\
\infty \\
\Xi \\
\Xi\end{array}$ & $\begin{array}{l}\oplus_{0} \\
\stackrel{0}{\vec{u}} \\
\stackrel{y}{1}\end{array}$ & 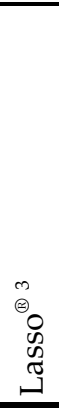 & 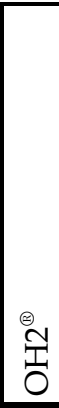 & 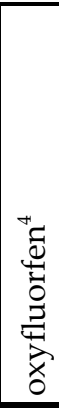 & 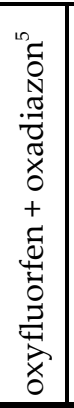 & 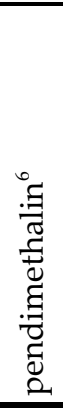 & 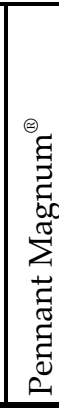 & 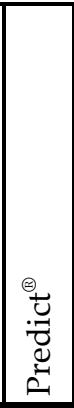 & 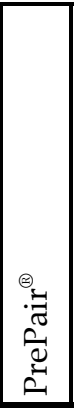 & 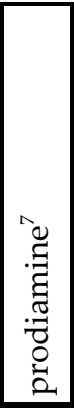 & 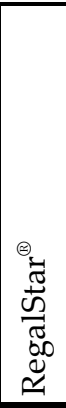 & 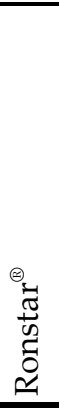 & $\begin{array}{l}\stackrel{\oplus}{*} \\
\stackrel{2}{\simeq} \\
\stackrel{1}{*}\end{array}$ & $\begin{array}{l}\tilde{y} \\
\text { J } \\
\vdots \\
3 \\
0 \\
\text { ऊ }\end{array}$ & 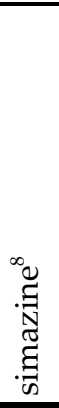 & 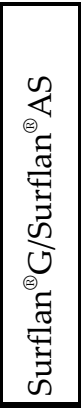 & 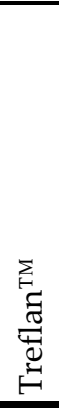 & 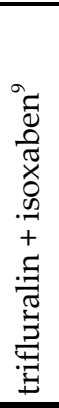 & 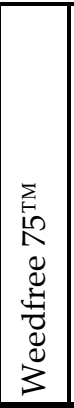 & $\stackrel{\oplus}{\times}$ \\
\hline Gladiolus spp. & $\mathrm{Y}^{11}$ & & $\mathrm{Y}$ & $\mathrm{Y}$ & & & & & & & & & & & $\mathrm{Y}$ & $\mathrm{Y}$ & & & $\mathrm{Y}$ & & $\mathrm{Y}$ & & & & $Y / Y^{11}$ & $\mathrm{Y}$ & & & $Y^{11}$ \\
\hline \multicolumn{30}{|l|}{ Gloriosa superba } \\
\hline Gomphrena cultivars & $\mathrm{Y}$ & & & & & & & & & & & & & & & & & & & & & & & & & & & & \\
\hline Gypsophila paniculata & & & $\mathrm{Y}$ & & & & & $\mathrm{Y}$ & & & & & & & $\mathrm{Y}$ & & & & $\mathrm{Y}^{11}$ & & $\mathrm{Y}$ & $\mathrm{Y}$ & & & $\mathrm{Y} / \mathrm{Y}$ & $\mathrm{Y}$ & $\mathrm{N}$ & & $\mathrm{Y}$ \\
\hline Helianthus spp. & & & $\mathrm{Y}$ & & & & & & & & & & & & & & & & & & & & & & & $\mathrm{Y}$ & & & \\
\hline Helichrysum bracteatum & & & $\mathrm{Y}$ & & & & & & & & & & & & & & & & & & & & & & & & & & \\
\hline Heliconia species & & & $\mathrm{Y}$ & & & & & & & & & & & & & & & & & & & & & & & & & & \\
\hline Hemerocallis spp. & & & & & & & & $\mathrm{Y}$ & & & & & & & $\mathrm{Y}$ & $\bar{Y}$ & & & $\mathrm{Y}$ & & & & & & $\mathrm{Y} / \mathrm{Y}$ & $\mathrm{Y}$ & $\mathrm{Y}$ & & $\mathrm{Y}$ \\
\hline Heuchera sanguinea & $\mathrm{Y}$ & & $\mathrm{Y}$ & & & & & $\mathrm{Y}$ & & & & & & & & & & & $\mathrm{Y}$ & & & & & & & $\mathrm{Y}$ & & & \\
\hline \multicolumn{30}{|l|}{ Hippeastrum cultivars } \\
\hline Hosta spp. & & & & $\mathrm{Y}$ & & $\mathrm{Y}$ & & $\mathrm{Y}^{11}$ & & & & & & & $\mathrm{Y}$ & $\mathrm{Y}^{11}$ & & & $\mathrm{Y}^{11}$ & & & & & & $\mathrm{Y} / \mathrm{Y}$ & $Y^{11}$ & $\mathrm{Y}^{11}$ & & $\mathrm{Y}$ \\
\hline Hyacinthus spp. & & & & & & & & & & & & & & & & $\mathrm{Y}$ & & & & & & & & & & & & & \\
\hline Iberis sempervirens & $\mathrm{Y}$ & & $\mathrm{Y}$ & & & $Y^{11}$ & & $\mathrm{~N}$ & & & & & & & & & & & & & $\mathrm{Y}$ & $\mathrm{Y}$ & & & & & $\mathrm{N}$ & & \\
\hline Impatiens walleriana & & & & & & $\mathrm{Y}$ & & & & & & & & & $\mathrm{Y}$ & $Y^{11}$ & & & & & & & & & $\mathrm{Y} / \mathrm{Y}$ & $\mathrm{Y}$ & & & $\mathrm{Y}$ \\
\hline Iris spp. & & & $\mathrm{Y}$ & & & $\mathrm{Y}$ & & $Y^{11}$ & & & & & & & $\mathrm{Y}$ & $\mathrm{Y}$ & & & $Y$ & & & & & & $\mathrm{Y} / \mathrm{Y}$ & $\mathrm{Y}$ & & & $\mathrm{Y}$ \\
\hline \multicolumn{30}{|l|}{ Kalanchoe cultivars } \\
\hline Kniphofia uvaria & & & $\mathrm{Y}$ & & & & & & & & & & & & & & & & & & & & & & & & & & \\
\hline Lathyrus odoratus & $\mathrm{Y}$ & & $\mathrm{Y}$ & & & & & & & & & & & & & & & & & & & & & & & & & & \\
\hline Liatris spicata & & & & & & & & & & & & & & & $\mathrm{Y}$ & & & & & & & & & & $\mathrm{Y} / \mathrm{Y}$ & $\mathrm{Y}$ & & & $\mathrm{Y}$ \\
\hline Lillium spp. & & & $\mathrm{Y}$ & & & & & & & & & & & & $\mathrm{Y}$ & $\mathrm{Y}$ & & & $\mathrm{Y}$ & & & & & & & & & & \\
\hline Limonium perezii & & & & & & & & $\mathrm{Y}$ & & & & & & & $\mathrm{Y}$ & & & & & & $\mathrm{Y}$ & & & & & $\mathrm{Y}$ & $\mathrm{Y}$ & & \\
\hline Lobelia spp. & & & & & & & & & & & & & & & & & & & $\mathrm{Y}^{11}$ & & & & & & & $\mathrm{Y}$ & & & \\
\hline \multicolumn{30}{|l|}{ Lunaria annua } \\
\hline Lupinus spp. & & & $\mathrm{Y}$ & & & & & & & & & & & & Y & $\mathrm{Y}$ & & & & & & & & & & $\mathrm{Y}$ & & & \\
\hline
\end{tabular}




\begin{tabular}{|c|c|c|c|c|c|c|c|c|c|c|c|c|c|c|c|c|c|c|c|c|c|c|c|c|c|c|c|c|c|}
\hline Scientific name & 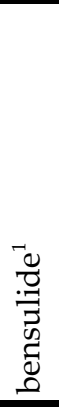 & 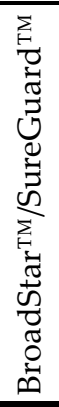 & 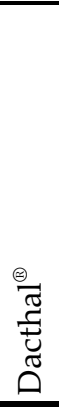 & 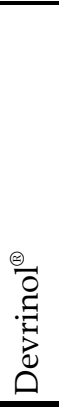 & 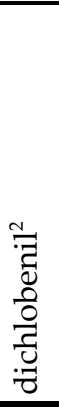 & 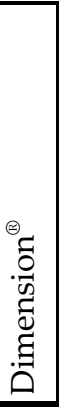 & 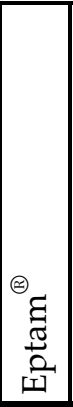 & 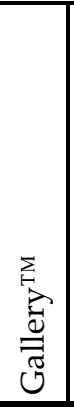 & $\begin{array}{l}\Theta_{0} \\
\infty \\
\Xi \\
\Xi \\
\Xi\end{array}$ & 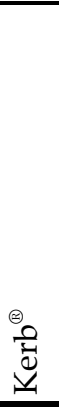 & 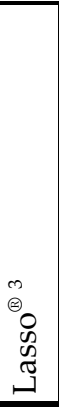 & $\begin{array}{l}\stackrel{\otimes}{2} \\
\stackrel{7}{0}\end{array}$ & 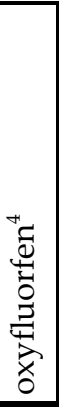 & 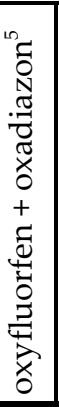 & 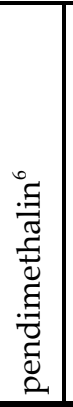 & 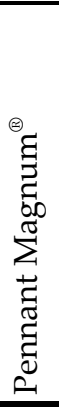 & 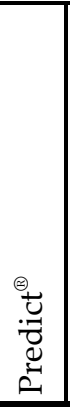 & 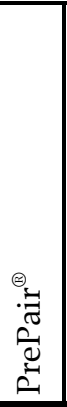 & 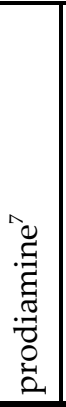 & 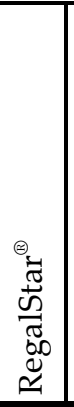 & 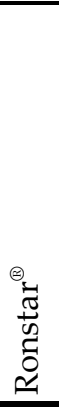 & \begin{tabular}{c}
$\Theta_{1}$ \\
\multirow{2}{*}{} \\
0 \\
$\simeq$
\end{tabular} & $\begin{array}{c}0 \\
\tilde{D} \\
\tilde{U} \\
3 \\
0 \\
0 \\
\tilde{c}\end{array}$ & 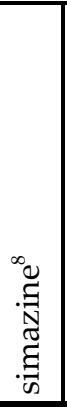 & 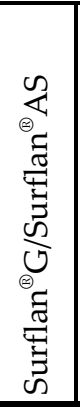 & 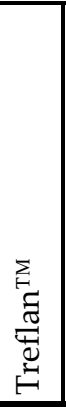 & 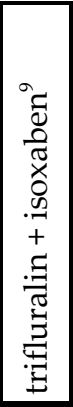 & 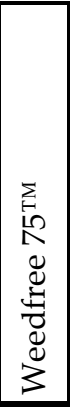 & 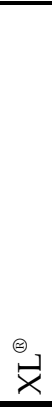 \\
\hline Lysimachia spp. & & & & & & & & $\mathrm{Y}$ & & & & & & & & & & & & & & & & & & & $\mathrm{Y}$ & & \\
\hline Mirabilis jalapa & & & $\mathrm{Y}$ & & & & & & & & & & & & & & & & & & & & & & & $\mathrm{Y}$ & & & \\
\hline Monarda didyma & & & & & & $\mathrm{Y}$ & & & & & & & & & & & & & & & & & & & & & & & \\
\hline Narcissus spp. & $\mathrm{Y}$ & & & $\mathrm{Y}$ & & $\mathrm{Y}$ & & $\mathrm{Y}$ & & & & & & & $\mathrm{Y}$ & $\mathrm{Y}$ & & & $\mathrm{Y}$ & & & & & & & $\mathrm{Y}$ & & & \\
\hline \multicolumn{30}{|l|}{ Nigella damascena } \\
\hline Paeonia cultivars & & & & & & & & & & & & & & & & & & & $\mathrm{Y}^{11}$ & $\mathrm{Y}$ & & $\mathrm{Y}$ & & & & & & & \\
\hline Pachysandra terminalis & $\mathrm{Y}$ & & & $\mathrm{Y}$ & & $\mathrm{Y}$ & & $\mathrm{Y}$ & & & & $\mathrm{Y}$ & & $\mathrm{Y}$ & $\mathrm{Y}$ & $\mathrm{Y}$ & & & & & $\mathrm{Y}$ & & & & $-/ Y$ & $\mathrm{Y}$ & $\mathrm{Y}$ & & \\
\hline Pelargonium hortorum & & & $\mathrm{Y}$ & $\mathrm{Y}$ & & $\mathrm{Y}$ & & & & & & & & & $\mathrm{Y}^{11}$ & $\mathrm{Y}$ & & & & & & & & & $\mathrm{Y} / \mathrm{Y}$ & & & & $\mathrm{Y}$ \\
\hline Pentas lanceolata & & & & & & & & $\mathrm{Y}$ & & & & & & & & & & & & & & & & & & & & & \\
\hline Petunia $\times$ hybrida & & & $\mathrm{Y}$ & $\mathrm{Y}$ & & $\mathrm{Y}$ & & $\mathrm{Y}$ & & & & & & & $\mathrm{Y}$ & $\mathrm{Y}$ & & & & & & & & & $\mathrm{Y} / \mathrm{Y}$ & $\mathrm{Y}$ & $\mathrm{Y}$ & & $\mathrm{Y}$ \\
\hline \multicolumn{30}{|l|}{ Phalaenopsis cultivars } \\
\hline Philodendron spp. & & & & & & & & & & & & $\mathrm{Y}$ & & Y & & & & & & & & & & & & & & & \\
\hline Phlox spp. & & & $\mathrm{N}$ & & & & & $\mathrm{Y}^{11}$ & & & & & & & $\mathrm{Y}$ & $\mathrm{Y}$ & & & & & & & & & & $\mathrm{Y}$ & $\mathrm{N}^{12}$ & & \\
\hline \multicolumn{30}{|l|}{ Platycodon grandiflorus } \\
\hline Portulaca grandiflora & & & $\mathrm{Y}$ & & & $\mathrm{Y}$ & & & & & & & & & $\mathrm{Y}$ & & & & & & & & & & $\mathrm{Y} / \mathrm{Y}$ & $\mathrm{Y}$ & & & $\mathrm{Y}$ \\
\hline Primula spp. & $\mathrm{Y}$ & & & & & & & & & & & & & & & & & & & & & & & & & & & & \\
\hline Protea spp. \& cultivars & & & & & & & & & & & & & & & & & & & & & $\mathrm{Y}$ & $\mathrm{Y}^{11}$ & & & $\mathrm{Y} / \mathrm{Y}^{11}$ & & & & \\
\hline Ranunculus asiaticus & $\mathrm{Y}$ & & & & & & & & & & & & & & & & & & & & & & & & & & & & $\mathrm{Y}$ \\
\hline Rosmarinus officinalis & & & & & & $\mathrm{Y}$ & & $\mathrm{Y}$ & & & & & & & & & & & & & & & & & $\mathrm{Y} / \mathrm{Y}$ & $\mathrm{Y}$ & $\mathrm{Y}$ & & Y \\
\hline Rudbeckia fulgida & & & & & & $\mathrm{Y}^{11}$ & & $\mathrm{Y}$ & & & & & & & $\mathrm{Y}^{11}$ & & & & $\mathrm{Y}^{11}$ & & & & & & $-/ Y$ & $\mathrm{Y}^{11}$ & $\mathrm{Y}^{11}$ & & $\mathrm{Y}$ \\
\hline Rudbeckia hirta & $\mathrm{Y}$ & & & $\mathrm{Y}$ & & & & & & & & & & & $\mathrm{Y}$ & & & & & & & & & & $\mathrm{Y} / \mathrm{Y}$ & $\mathrm{Y}$ & $\mathrm{Y}$ & & $\mathrm{Y}$ \\
\hline Saintpaulia ionantha & & & $\mathrm{Y}$ & & & & & & & & & & & & & & & & & & & & & & & & & & \\
\hline Salvia farinacea & & & & & & $\mathrm{Y}$ & & & & & & & & & $\mathrm{Y}^{11}$ & & & & & & & $\mathrm{Y}$ & & & & $\mathrm{Y}$ & & & $\mathrm{Y}$ \\
\hline Salvia spp. & & & $\mathrm{Y}$ & & & & & & & & & & & & & & & & & & & $\mathrm{Y}$ & & & $\mathrm{Y} / \mathrm{Y}$ & $\mathrm{Y}$ & & & $\mathrm{Y}$ \\
\hline
\end{tabular}




\begin{tabular}{|c|c|c|c|c|c|c|c|c|c|c|c|c|c|c|c|c|c|c|c|c|c|c|c|c|c|c|c|c|c|}
\hline Scientific name & 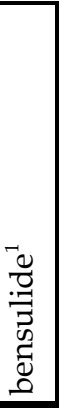 & 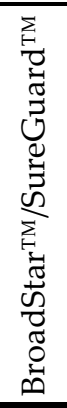 & 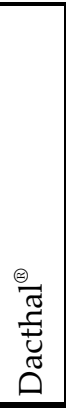 & 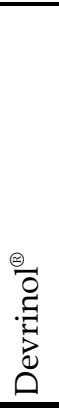 & 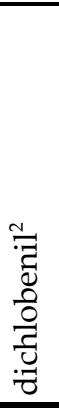 & 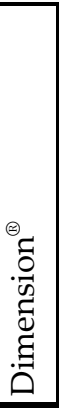 & 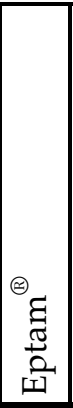 & 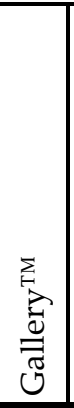 & $\begin{array}{l}\Theta_{0} \\
\stackrel{\infty}{\Xi} \\
\Xi \\
\Xi\end{array}$ & 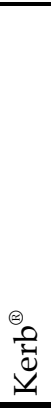 & 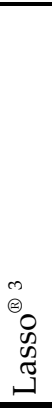 & $\begin{array}{l}\stackrel{\oplus}{1} \\
\stackrel{1}{O}\end{array}$ & 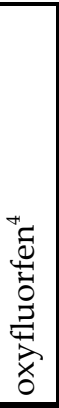 & 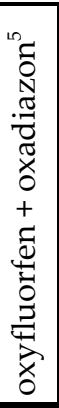 & 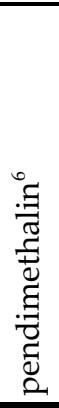 & 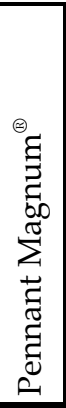 & 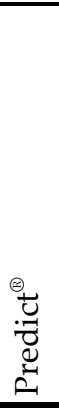 & 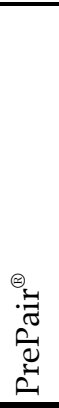 & 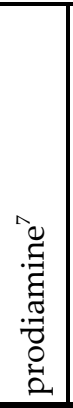 & 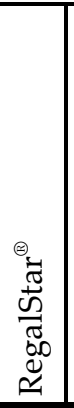 & 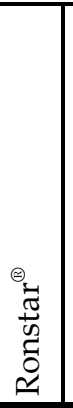 & $\begin{array}{l}\Theta_{1} \\
\vec{z} \\
\stackrel{0}{*}\end{array}$ & 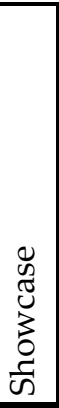 & 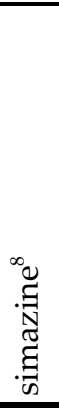 & 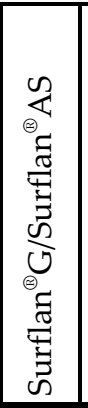 & 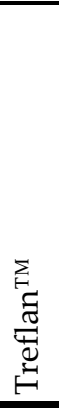 & 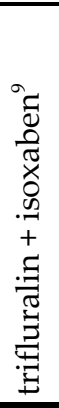 & 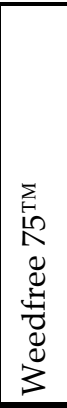 & $\stackrel{\oplus}{\ominus}_{\vec{x}}$ \\
\hline Scabiosa cultivars & & & & & & & & & & & & & & & & & & & & & & & & & & $\mathrm{Y}$ & & & \\
\hline Schefflera arboricola & & & & & & & & & & & & & & & & & & & & & & $\mathrm{Y}$ & & & & & & & \\
\hline \multicolumn{30}{|l|}{ Scilla siberica } \\
\hline Sedum spp. & $\mathrm{Y}$ & & & $\mathrm{Y}$ & & $\mathrm{Y}$ & & $\mathrm{N}$ & & & & & & & $\mathrm{Y}$ & $\mathrm{Y}$ & & $\mathrm{Y}$ & $\mathrm{Y}^{11}$ & & $\mathrm{Y}$ & & & & $\mathrm{Y} / \mathrm{Y}^{11}$ & $\mathrm{Y}$ & $\mathrm{N}$ & & $Y^{11}$ \\
\hline Senecio cineraria & & & & & & $\mathrm{Y}$ & & & & & & & & & $\mathrm{Y}$ & $\mathrm{Y}^{11}$ & & & & & & & & & & & & & \\
\hline \multicolumn{30}{|l|}{ Solidago cultivars } \\
\hline Strelitzia reginae & & & & $\mathrm{Y}$ & & & & & & & & & & & & & & & & & & & & & $\mathrm{Y} / \mathrm{Y}$ & & & & $\mathrm{Y}$ \\
\hline Tagetes spp. & $\mathrm{Y}$ & & $\mathrm{Y}$ & & & $\mathrm{Y}$ & & & & & & & & & $\mathrm{Y}^{11}$ & $\mathrm{Y}$ & & & & & & & & & $\mathrm{Y} / \mathrm{Y}$ & $\mathrm{Y}$ & & & $\mathrm{Y}$ \\
\hline \multicolumn{30}{|l|}{ Trachelium caeruleum } \\
\hline Tradescantia virginiana & & & $\mathrm{Y}$ & & & & & & & & & & & & & & & & & & & & & & & & & & \\
\hline Tulbaghia violacea & & & & & & & & $\mathrm{Y}$ & $\mathrm{Y}$ & & & & & & & & & & & & & & & & $-/ Y$ & & $\mathrm{Y}$ & & \\
\hline Tulipa spp. & $\mathrm{Y}$ & & & & & & & $\mathrm{Y}$ & & & & & & & $\mathrm{Y}$ & $\mathrm{Y}$ & & & $\mathrm{Y}$ & & & & & & $\mathrm{Y} / \mathrm{Y}$ & $\mathrm{Y}$ & & & $\mathrm{Y}$ \\
\hline Verbena spp. & & & & & & & & $\mathrm{Y}$ & & & & & & & $\mathrm{Y}^{11}$ & & & & & & & $Y^{11}$ & & & $-/ Y^{11}$ & $\mathrm{Y}$ & & & \\
\hline Vinca spp. & $\mathrm{Y}$ & & & $\mathrm{Y}$ & & $\mathrm{Y}^{11}$ & & $\mathrm{Y}$ & & & & $\mathrm{Y}$ & & $\mathrm{Y}$ & $\mathrm{Y}^{11}$ & $\mathrm{Y}^{11}$ & & & $\mathrm{Y}$ & $\mathrm{Y}$ & $\mathrm{Y}$ & & & & $\mathrm{Y} / \mathrm{Y}^{11}$ & $\mathrm{Y}$ & $\mathrm{Y}$ & & $\mathrm{Y}$ \\
\hline Viola spp. & $\mathrm{Y}$ & & $\mathrm{N}$ & & & $\mathrm{Y}^{11}$ & & & & & & & & & $\mathrm{Y}^{11}$ & $\mathrm{Y}$ & & & & & & & & & $\mathrm{Y} / \mathrm{Y}^{11}$ & & & & $Y^{11}$ \\
\hline \multicolumn{30}{|l|}{$\begin{array}{l}\text { Xerochrysum bracteatum } \\
\text { (see Helichrysum } \\
\text { bracteatum) }\end{array}$} \\
\hline Yucca filamentosa & & & & & & & & $\mathrm{Y}$ & & & & $\mathrm{N}$ & & & $\mathrm{Y}$ & $\mathrm{Y}^{11}$ & & & $\mathrm{Y}$ & & & & & & & $\mathrm{Y}$ & $\mathrm{Y}$ & & \\
\hline \multicolumn{30}{|l|}{ Zantedeschia cultivars } \\
\hline Zinnia elegans & $\mathrm{Y}$ & & $\mathrm{Y}$ & & & & & & & & & & & & $\mathrm{Y}$ & $\mathrm{Y}^{11}$ & & & & & & & & & $\mathrm{Y} / \mathrm{Y}$ & $\mathrm{Y}$ & & & $\mathrm{Y}$ \\
\hline
\end{tabular}




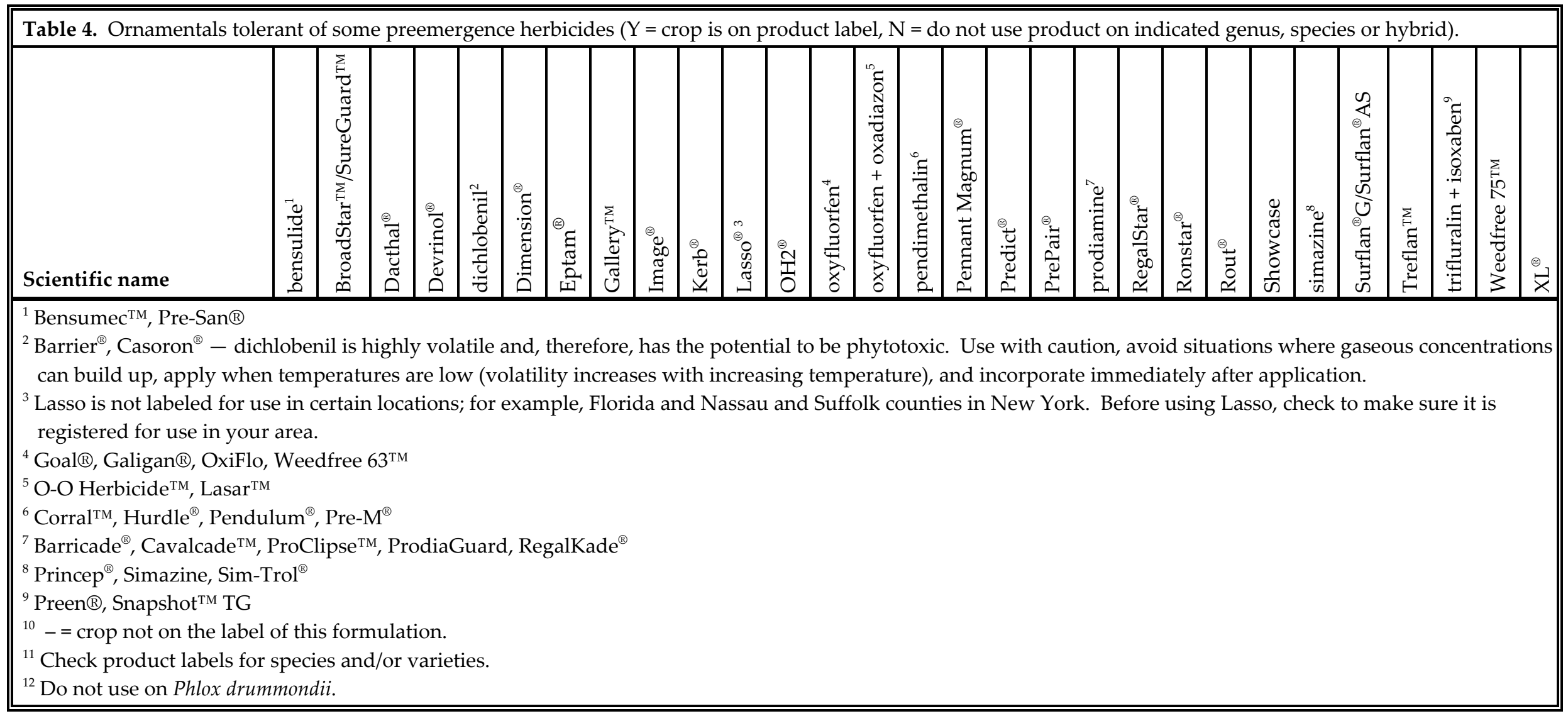




\begin{tabular}{|l|l|}
\hline Table 5. Common name reference list for ornamental crops. \\
\hline Common name & Scientific name \\
\hline abelia, glossy & Abelia grandiflora \\
\hline acacia & Acacia spp. \\
\hline Adam's needle, yucca & Yucca filamentosa \\
\hline African iris & $\begin{array}{l}\text { Dietes iridioides (synonym Dietes } \\
\text { vegeta) }\end{array}$ \\
\hline African violet & Saintpaulia ionantha \\
\hline agapanthus & Agapanthus africanus \\
\hline ageratum & Ageratum spp. \\
\hline ajuga & Ajuga reptans \\
\hline Algerian ivy & Hedera canariensis \\
\hline allamanda & Allamanda cathartica \\
\hline allium & Allium spp. \\
\hline alyssum & Alyssum spp. \\
\hline amaryllis & Hippeastrum cultivars \\
\hline American arborvitae & Thuja occidentalis \\
\hline American dogwood & Cornus stolonifera \\
\hline American holly & Ilex opaca \\
\hline American plane tree & Platanus occidentalis \\
\hline angelwing jasmine & Jasminum nitidum \\
\hline anise, Chinese & Illicium anisatum \\
\hline anise, Florida & Illicium floridanum \\
\hline anise, purple & Illicium floridanum \\
\hline anise, star & Illicium parviflorum \\
\hline anthurium & Anthurium andraeanum \\
\hline aralia, Japanese & Fatsia japonica \\
\hline arborvitae, American & Thuja occidentalis \\
\hline arborvitae, Oriental & $\begin{array}{l}\text { Platycladus orientalis (synonym } \\
\text { Thuja orientalis) }\end{array}$ \\
\hline areca palm & Chrysalidocarpus lutescens \\
\hline Arizona cypress & Cupressus arizonica \\
\hline Preamergence Hericis for Us in \\
\hline
\end{tabular}

Preemergence Herbicides for Use in Ornamentals

\begin{tabular}{|l|l|}
\hline \multicolumn{2}{|l|}{ Table 5. Common name reference list for ornamental crops. } \\
\hline Common name & Scientific name \\
\hline arum lily & Zantedeschia cutlivars \\
\hline ash & Fraxinus spp. \\
\hline asparagus "fern" & Asparagus spp \\
\hline aster & Aster spp. \\
\hline astilbe & Astilbe spp \\
\hline aucuba, gold-dust plant & Aucuba japonica \\
\hline Australian tree fern & Cyathea australis \\
\hline azalea species & Rhododendron spp. \\
\hline baby's breath & Gypsophila paniculata \\
\hline bachelor's button & Gomphrena cultivars \\
\hline bald cypress & Taxodium distichum \\
\hline balloon flower & Platycodon grandiflorus \\
\hline bamboo, heavenly & Nandina domestica \\
\hline bamboo palm & Chamaedorea erumpens \\
\hline bamboo palm & Rhapis excelsa \\
\hline banana & Musa aluminata \\
\hline banana shrub & Michelia figo \\
\hline barberry & Berberis spp. \\
\hline barberry & Berberis $\times$ gladwynensis \\
\hline barberry, Japanese & Berberis thunbergii \\
\hline bee balm & Monarda didyma \\
\hline begonia & Begonia spp. \\
\hline bellflower & Campanula spp. \\
\hline bergamot & Monarda didyma \\
\hline birch, river & Betula nigra \\
\hline bird-of-paradise & Strelitzia reginae \\
\hline bishop's flower & Ammi majus \\
\hline black gum & Nyssa sylvatica \\
\hline black-eyed Susan & \\
\hline
\end{tabular}

Preemergence Herbicides for Use in Ornamentals 


\begin{tabular}{|c|c|}
\hline Common name & Scientific name \\
\hline blanket flower & Gaillardia spp. \\
\hline blazing star & Liatris spicata \\
\hline blue lace flower & Trachelium caeruleum \\
\hline blue Pacific juniper & Juniperus conferta \\
\hline blue star juniper & Juniperus squamata \\
\hline blue throatwort & Trachelium caeruleum \\
\hline bluebeard & Caryopteris incana \\
\hline bottlebrush, lemon & Callistemon citrinus \\
\hline bottlebrush, weeping & Callistemon viminalis \\
\hline bougainvillea & Bougainvillea spp. \\
\hline boxwood & Buxus spp. \\
\hline boxwood, littleleaf & Buxus microphylla \\
\hline Bradford pear & Pyrus calleryana 'Bradford' \\
\hline buckeye, red & Aesculus pavia \\
\hline bush, butterfly & $\begin{array}{l}\text { Buddleja davidii, (synonym Buddleia } \\
\text { davidii) }\end{array}$ \\
\hline bush lantana & Lantana camara \\
\hline butcher's broom & Ruscus hypophyllum \\
\hline buttercup & Ranunculus asiaticus \\
\hline butterfly bush & $\begin{array}{l}\text { Buddleja davidii, (synonym Buddleia } \\
\text { davidii) }\end{array}$ \\
\hline butterfly weed & Asclepias tuberosa \\
\hline caladium & Caladium bicolor \\
\hline calla lily & Zantedeschia cutlivars \\
\hline camellia & Camellia spp. \\
\hline camellia, Japanese & Camellia japonica \\
\hline camellia, sasanqua & Camellia sasanqua \\
\hline Canary Island pine & Pinus canariensis \\
\hline candytuft, evergreen & Iberis sempervirens \\
\hline
\end{tabular}

\begin{tabular}{|c|c|}
\hline Common name & Scientific name \\
\hline cape jasmine or jessamine & $\begin{array}{l}\text { Gardenia augusta (synonym G. } \\
\text { jasminoides) }\end{array}$ \\
\hline carnation & Dianthus caryophyllus \\
\hline Carolina jessamine & Gelsemium sempervirens \\
\hline cast iron plant & Aspidistra elatior \\
\hline cedar, Eastern red & Juniperus virginiana \\
\hline cedar, Southern red & Juniperus silicicola \\
\hline chalcus & Murraya paniculata \\
\hline cherry & Prunus spp. \\
\hline cherry laurel, American & Prunus caroliniana \\
\hline cherry laurel, English & Prunus laurocerasus \\
\hline chestnut & Castanea spp. \\
\hline chestnut oak & Quercus prinus \\
\hline Chinese anise & Illicium anisatum \\
\hline Chinese elm & Ulmus parvifolia \\
\hline Chinese fan palm & Livistona chinensis \\
\hline Chinese holly & Ilex cornuta \\
\hline Chinese juniper & Juniperus chinensis \\
\hline Chinese privet, variegated & Ligustrum sinense 'Variegated' \\
\hline Christmas palm & Veitchia merrillii \\
\hline chrysanthemum & Chrysanthemum spp. \\
\hline chrysanthemum & Dendranthema $\times$ grandiflorum \\
\hline citrus, ornamental & Citrus spp. \\
\hline cleyera & $\begin{array}{l}\text { Cleyera japonica, see also } \\
\text { Ternstroemia gymnanthera }\end{array}$ \\
\hline climbing lily & $\begin{array}{l}\text { Gloriosa rothschildiana, see G. superba } \\
\text { 'Rothschildiana }\end{array}$ \\
\hline coastal leucothoe & Leucothoe axillaris \\
\hline cockscomb, cock's comb & Celosia cristata \\
\hline coleus & Coleus spp. \\
\hline
\end{tabular}

\section{Preemergence Herbicides for Use in Ornamentals}

Preemergence Herbicides for Use in Ornamentals 


\begin{tabular}{|l|l|}
\hline \multicolumn{2}{|l|}{ Table 5. Common name reference list for ornamental crops. } \\
\hline Common name & Scientific name \\
\hline columbine & Aquilegia cultivars \\
\hline coneflower & Echinacea purpurea \\
\hline Confederate jasmine & Trachelospermum jasminoides \\
\hline copperleaf & Acalypha wilkesiana \\
\hline coral bells & Heuchera sanguinea \\
\hline coreopsis & Coreopsis spp. \\
\hline cosmos & Cosmos spp. \\
\hline cotoneaster & Cotoneaster spp. \\
\hline cotoneaster, cranberry & Cotoneaster apiculatus \\
\hline cottonwood, Eastern & Populus deltoides \\
\hline crape myrtle & Lagerstroemia indica \\
\hline creeping juniper & Juniperus horizontalis \\
\hline croton & Codiaeum variegatum \\
\hline cypress, Arizona & Cupressus arizonica \\
\hline cypress, bald & Taxodium distichum \\
\hline cypress, false & Chamaecyparis spp. \\
\hline cypress, Hinoki & Chamaecyparis obtusa \\
\hline cypress, Italian & C. sempervirens \\
\hline cypress, Lawson's & Chamaecyparis lawsoniana \\
\hline cypress, Leyland & $\times$ Cupressocyparis leylandii \\
\hline cypress, Sawara & Chamaecyparis pisifera \\
\hline cypress spp. & Cupressus spp. \\
\hline daffodil & bulbs, ornamental \\
\hline daffodil & Narcissus spp. \\
\hline dahlia & Dahlia spp. \\
\hline daisy, gloriosa & Rudbeckia hirta \\
\hline daisy, shasta & Chrysanthemum superbum \\
\hline date palm, pigmy & Phoenix roebelinii \\
\hline daylily & Hemerocallis spp. \\
\hline
\end{tabular}

\begin{tabular}{|l|l|}
\hline \multicolumn{2}{|l|}{ Table 5. Common name reference list for ornamental crops. } \\
\hline Common name & Scientific name \\
\hline delphinium & Delphinium spp. \\
\hline deutzia & Deutzia spp. \\
\hline dianthus & Dianthus plumarius \\
\hline dogwood, American & Cornus stolonifera \\
\hline dogwood, flowering & Cornus florida \\
\hline dogwood, kousa & Cornus kousa \\
\hline dracaena & Dracaena spp. \\
\hline drake elm & Ulmus parvifolia \\
\hline drooping leucothoe & Leucothoe fontanesiana \\
\hline dusty miller & Senecio cineraria \\
\hline Eastern cottonwood & Populus deltoides \\
\hline Eastern red cedar & Juniperus virginiana \\
\hline Eastern white pine & Pinus strobus \\
\hline elaeagnus, thorny & Elaeagnus pungens \\
\hline elm & Ulmus spp. \\
\hline elm, Chinese & Ulmus parvifolia \\
\hline elm, drake & Ulmus parvifolia \\
\hline English ivy & Hedera helix \\
\hline escallonia & Escallonia spp. \\
\hline eucalyptus & Eucalyptus spp. \\
\hline eucalyptus, silver dollar & Eucalyptus cinerea \\
\hline euonymous & Euonymous spp. \\
\hline euonymous, creeping & Euonymus fortunei \\
\hline euonymus, evergreen & Euonymus japonica \\
\hline euonymus, spreading & Euonymus kiautshovicus \\
\hline European fan palm & Chamaerops humilis \\
\hline false bird of Paradise & Heliconia species \\
\hline false cypress & Chamaecyparis spp. \\
\hline false holly & Osmanthus heterophyllus \\
\hline
\end{tabular}




\begin{tabular}{|l|l|}
\hline \multicolumn{2}{|l|}{ Table 5. Common name reference list for ornamental crops. } \\
\hline Common name & Scientific name \\
\hline false saffron & Carthamus tinctorius \\
\hline false spiraea & Astilbe spp. \\
\hline false spiraea & Astilbe cultivars \\
\hline fan palm, Chinese & Livistona chinensis \\
\hline fan palm, European & Chamaerops humilis \\
\hline fan palm, Mexican & Washingtonia robusta \\
\hline fan palm, Washington & Washingtonia robusta \\
\hline fatshedera & $\times$ Fatshedera lizei \\
\hline “fern", asparagus & Asparagus spp. \\
\hline fern, Australian tree & Cyathea australis \\
\hline fern, Japanese painted & Athyrium nipponicum \\
\hline fern, leatherleaf & Rumohra adiantiformis \\
\hline “fern", tree & Asparagus virgatus \\
\hline ficus & Ficus spp. \\
\hline ficus, mini & Ficus benjamina \\
\hline fiddleleaf fig & Ficus lyrata \\
\hline fig, fiddleleaf & Ficus lyrata \\
\hline fig, weeping & Ficus benjamina \\
\hline firethorn & Pyracantha spp. \\
\hline firethorn, Formosa & Pyracantha koidzumii \\
\hline firethorn, scarlet & Pyracantha coccinea \\
\hline fishtail palm & Caryota spp. \\
\hline flame lily & Gloriosa superba \\
\hline flame maple & Acer ginnala \\
\hline fleabane & Erigeron cultivars \\
\hline Florida anise & Illicium floridanum \\
\hline florists chrysanthemum & Dendranthema $\times$ grandiflorum \\
\hline florists' cyclamen & Cyclamen persicum \\
\hline flowering dogwood & Cornus florida \\
\hline
\end{tabular}

\begin{tabular}{|l|l|}
\hline Table 5. Common name reference list for ornamental crops. \\
\hline Common name & Scientific name \\
\hline forsythia & Forsythia spp. \\
\hline forsythia, border & Forsythia $\times$ intermedia \\
\hline Fortune's osmanthus & Osmanthus $\times$ fortunei \\
\hline fountain grass & Pennisetum spp. \\
\hline four o'clock & Mirabilis jalapa \\
\hline foxglove & Digitalis spp. \\
\hline Fraser's photinia & Photinia $\times$ fraseri \\
\hline freesia & Freesia spp. \\
\hline gaillardia & Gaillardia spp. \\
\hline gallberry & Ilex glabra \\
\hline gardenia & Gardenia jasminoides \\
\hline garlic, society & Tulbaghia violacea \\
\hline gazania & Gazania spp. \\
\hline geranium & Pelargonium hortorum \\
\hline gerbera daisy & Gerbera jamesonii \\
\hline ginko & Ginko biloba \\
\hline gladiolus & Gladiolus spp. \\
\hline globe amaranth & Gomphrena cultivars \\
\hline gloriosa daisy & Rudbeckia hirta \\
\hline gloriosa lily & Gloriosa rothschildiana, see G. superba \\
\hline 'Rothschildiana \\
\hline glory lily & Gloriosa superba \\
\hline glossy abelia & Abelia $\times$ grandiflora \\
\hline glossy privet & Ligustrum lucidum \\
\hline gold-dust plant & Aucuba japonica \\
\hline golden everlasting & Helichrysum bracteatum \\
\hline goldenrod & Solidago species \& cultivars \\
\hline grass, fountain & Pennisetum spp. \\
\hline grass, mondo & Ophiopogon japonicus \\
\hline
\end{tabular}




\begin{tabular}{|l|l|}
\hline \multicolumn{2}{|l|}{ Table 5. Common name reference list for ornamental crops. } \\
\hline Common name & Scientific name \\
\hline grass, pampas & Cortaderia selloana. \\
\hline guava, pineapple & Acca sellowiana \\
\hline guava, strawberry & Psidium littorale var. lucidum \\
\hline gum, black & Nyssa sylvatica \\
\hline gum, lemon scented & Eucalyptus citriodora \\
\hline gum, sweet & Liquidambar styraciflua \\
\hline hawthorn, Indian & Rhaphiolepis umbellata \\
\hline hawthorn, Indian & Rhaphiolepis indica \\
\hline heather, Mexican & Cuphea hyssopifolia \\
\hline heather, Scotch & Calluna vulgaris \\
\hline heavenly bamboo & Nandina domestica \\
\hline hibiscus & Hibiscus rosa-sinensis \\
\hline hickory & Carya spp. \\
\hline Hinoki cypress & Chamaecyparis obtusa \\
\hline holly & Ilex spp. \\
\hline holly, American & Ilex opaca \\
\hline holly, Chinese & Ilex cornuta \\
\hline holly, false & Osmanthus heterophyllus \\
\hline holly, Japanese & Ilex crenata \\
\hline holly, Nellie Stevens & Ilex $\times$ 'Nellie Stevens' \\
\hline holly, Savannah & Ilex attenuata 'Savannah' \\
\hline holly, yaupon & Ilex vomitoria \\
\hline honesty & Lunaria annua \\
\hline honeysuckle & Lonicera spp. \\
\hline honeysuckle, Japanese & Lonicera japonica \\
\hline hosta & Hosta spp. \\
\hline hyacinth & bulbs, ornamental \\
\hline hyacinth & Hyacinthus spp. \\
\hline hydrangea & Hydrangea spp. \\
\hline
\end{tabular}

\begin{tabular}{|l|l|}
\hline Table 5. Common name reference list for ornamental crops. \\
\hline Common name & Scientific name \\
\hline impatiens & Impatiens walleriana \\
\hline Indian hawthorn & Rhaphiolepis indica \\
\hline inkberry & Ilex glabra \\
\hline iris & Iris spp. \\
\hline iris, African & $\begin{array}{l}\text { Dietes iridioides (synonym Dietes } \\
\text { vegeta) }\end{array}$ \\
\hline iris, bulbous & bulbs, ornamental \\
\hline Italian cypress & Cupressus sempervirens \\
\hline ivy, Algerian & Hedera canariensis \\
\hline ivy, English & Hedera helix \\
\hline ixora & Ixora coccinea \\
\hline Jack pine & Pinus banksiana \\
\hline Japanese aralia & Fatsia japonica \\
\hline Japanese barberry & Berberis thunbergii \\
\hline Japanese camellia & Camellia japonica \\
\hline Japanese holly & Ilex crenata \\
\hline Japanese honeysuckle & Lonicera japonica \\
\hline Japanese maple & Acer palmatum \\
\hline Japanese painted fern & Athyrium nipponicum \\
\hline Japanese privet & Ligustrum japonicum \\
\hline Japanese spiraea & Spiraea japonica \\
\hline Japanese star jasmine & Trachelospermum asiaticum \\
\hline Japanese thistle & Cirsium japonicum \\
\hline Japanese viburnum & Viburnum japonicum \\
\hline jasmine & Jasminum spp. \\
\hline jasmine, angelwing & Jasminum nitidum \\
\hline jasmine, Confederate & Trachelospermum jasminoides \\
\hline jasmine, Japanese star & Trachelospermum asiaticum \\
\hline jasmine, orange & Murraya paniculata \\
\hline jasmine, pinwheel & Jasminum nitidum \\
\hline & \\
\hline
\end{tabular}

Preemergence Herbicides for Use in Ornamentals

Preemergence Herbicides for Use in Ornamentals 


\begin{tabular}{|l|l|}
\hline \multicolumn{2}{|l|}{ Table 5. Common name reference list for ornamental crops. } \\
\hline Common name & Scientific name \\
\hline jasmine, primrose & Jasminum mesnyi \\
\hline jasmine, shining & Jasminum nitidum \\
\hline jasmine, small-leaf & Trachelospermum asiaticum \\
\hline jasmine, star & Trachelospermum jasminoides \\
\hline jasmine, winter & Jasminum nudiflorum \\
\hline jessamine, Carolina & Gelsemium sempervirens \\
\hline juniper & Juniperus spp. \\
\hline juniper, blue Pacific & Juniperus conferta \\
\hline juniper, blue star & Juniperus squamata \\
\hline juniper, Chinese & Juniperus chinensis \\
\hline juniper, creeping & Juniperus horizontalis \\
\hline juniper, Japanese garden & Juniperus chinensis \\
\hline juniper, nana-dwarf & Juniperus procumbens \\
\hline juniper, Parson's, & Juniperus squamata \\
\hline juniper, savin & Juniperus sabina \\
\hline juniper, shore & Juniperus conferta \\
\hline kousa dogwood & Cornus kousa \\
\hline lady palm & Rhapis excelsa \\
\hline lantana, bush & Lantana camara \\
\hline lantana, trailing & Lantana sellowiana \\
\hline laurel, American cherry & Prunus caroliniana \\
\hline laurel, English cherry & Prunus laurocerasus \\
\hline laurel oak & Quercus laurifolia \\
\hline Lawson's cypress & Chamaecyparis lawsoniana \\
\hline leatherleaf fern & Rumohra adiantiformis \\
\hline lemon bottlebrush & Callistemon citrinus \\
\hline lemon scented gum & Eucalyptus citriodora \\
\hline leucothoe, coastal & Leucothoe axillaries \\
\hline leucothoe, drooping & $\times$ Cupressocyparis leylandii \\
\hline Leyland cypress & Ormamoe fontanesiana \\
\hline
\end{tabular}

Preemergence Herbicides for Use in Ornamentals

\begin{tabular}{|l|l|}
\hline Table 5. Common name reference list for ornamental crops. \\
\hline Common name & Scientific name \\
\hline liatris & Liatris spicata \\
\hline ligustrum & Ligustrum spp. \\
\hline lily & Lillium spp. \\
\hline lily of the Incas & Alstroemeria cultivars \\
\hline lily turf & Liriope muscari \\
\hline lily turf, creeping & Liriope spicata \\
\hline liroipe & Liriope muscari \\
\hline lisianthus & Eustoma grandiflorum \\
\hline littleleaf boxwood & Buxus microphylla \\
\hline live oak & Quercus virginiana \\
\hline lobelia & Lobelia spp. \\
\hline loblolly pine & Pinus taeda \\
\hline lobster claw & Heliconia species \\
\hline lodgepole pine & Pinus contorta \\
\hline longleaf pine & Pinus palustrus \\
\hline loquat & Eriobotrya japonica \\
\hline love-in-a-mist & Nigella damascena \\
\hline love-lies-bleeding & Amaranthus caudatus \\
\hline lupine & Lupinus spp. \\
\hline magnolia & Magnolia spp. \\
\hline magnolia, saucer & Magnolia $\times$ soulangiana \\
\hline magnolia, Southern & Magnolia grandiflora \\
\hline mahogany & Swietenia mahogani \\
\hline mahonia & Mahonia spp. \\
\hline Manila palm & Veitchia merrillii \\
\hline maple & Acer spp. \\
\hline maple, flame & Acer ginnala \\
\hline maple, Japanese & Acer palmatum \\
\hline maple, red & Acer rubrum \\
\hline maple, silver & Acer saccharinum \\
\hline
\end{tabular}

Preemergence Herbicides for Use in Ornamentals 


\begin{tabular}{|l|l|}
\hline Table 5. Common name reference list for ornamental crops. \\
\hline Common name & Scientific name \\
\hline maple, sugar & Acer saccharum \\
\hline marigold & Tagetes spp. \\
\hline Mexican fan palm & Washingtonia robusta \\
\hline Mexican heather & Cuphea hyssopifolia \\
\hline mondo grass & Ophiopogon japonicus \\
\hline money plant & Lunaria annua \\
\hline moneywort & Lysimachia nummularia \\
\hline moss rose & Portulaca grandiflora \\
\hline moth orchid & Phalaenopsis cultivars \\
\hline mulberry, white & Morus alba \\
\hline myrtle & Myrtus communis \\
\hline myrtle, crape & Lagerstroemia indica \\
\hline myrtle, wax & Myrica cerifera \\
\hline nana-dwarf juniper & Juniperus procumbens \\
\hline nandina & Nandina domestica \\
\hline narcissus & Narcissus spp. \\
\hline natal plum & $\begin{array}{l}\text { Carissa macrocarpa (synonym C. } \\
\text { grandiflora) }\end{array}$ \\
\hline Nellie Stevens holly & Ilex 'Nellie Stevens' $^{\prime}$ \\
\hline no-azami & Cirsium japonicum \\
\hline Norfolk Island pine & Araucaria heterophylla \\
\hline oak & Quercus spp. \\
\hline oak, chestnut & Quercus prinus \\
\hline oak, laurel & Quercus laurifolia \\
\hline oak, live & Quercus virginiana \\
\hline oak, pin & Quercus palustris \\
\hline oak, red & Quercus rubra \\
\hline oak, Shumard & Quercus shumardii \\
\hline oak, water & Quercus nigra \\
\hline oak, willow & Quercus phellos \\
\hline & \\
\hline
\end{tabular}

\begin{tabular}{|l|l|}
\hline \multicolumn{2}{|l|}{ Table 5. Common name reference list for ornamental crops. } \\
\hline Common name & Scientific name \\
\hline oak-leaf hydrangea & Hydrangea quercifolia \\
\hline old-fashioned weigela & Weigela florida \\
\hline oleander & Nerium oleander \\
\hline olive, Russian & Elaeagnus angustifolia \\
\hline orange jasmine & Murraya paniculata \\
\hline orchid tree & Bauhinia galpinii \\
\hline Oriental arborvitae & Platycladus orientalis (synonym \\
\hline osmanthus & Thuja orientalis) \\
\hline osmanthus, Fortune's & Osmanthus fragrans \\
\hline pachysandra & Osmanthus $\times$ fortunei \\
\hline painted fern, Japanese & Pachysandra terminalis \\
\hline palm, areca & Athyrium nipponicum \\
\hline palm, bamboo & Chrysalidocarpus lutescens \\
\hline palm, cat & Rhapis excelsa \\
\hline palm, Chinese fan & Chamaedorea cataractarum \\
\hline palm, Christmas & Livistona chinensis \\
\hline palm, European fan & Veitchia merrillii \\
\hline palm, fishtail & Chamaerops humilis \\
\hline palm, lady & Caryota spp. \\
\hline palm, Manila & Rhapis excelsa \\
\hline palm, Mexican fan & Veitchia merrillii \\
\hline palm, parlor & Washingtonia robusta \\
\hline palm, pigmy date & Chamaedorea elegans \\
\hline palm, queen & Phoenix roebelinii \\
\hline palm, sago & Syagrus romanzoffianum \\
\hline palm, windmill & Cycas revoluta \\
\hline palm, Washington fan & Trachycarpus fortunei \\
\hline pampas grass & Washingtonia robusta \\
\hline pansy & Cortaderia selloana. \\
\hline & Viola spp. \\
\hline
\end{tabular}

Preemergence Herbicides for Use in Ornamentals

Preemergence Herbicides for Use in Ornamentals 


\begin{tabular}{|l|l|}
\hline \multicolumn{2}{|l|}{ Table 5. Common name reference list for ornamental crops. } \\
\hline Common name & Scientific name \\
\hline parlor palm & Chamaedorea elegans \\
\hline Parson's juniper & Juniperus squamata \\
\hline pear, Bradford & Pyrus calleryana 'Bradford' \\
\hline pentas & Pentas lanceolata \\
\hline peony & Paeonia cultivars \\
\hline periwinkle, common & Vinca spp. \\
\hline periwinkle, Madagascar & Catharanthus roseus \\
\hline Persian buttercup & Ranunculus asiaticus \\
\hline Persian violet & Cyclamen persicum \\
\hline Peruvian lily & Alstroemeria cultivars \\
\hline petunia & Petunia $\times$ hybrida \\
\hline philodendron & Philodendron spp. \\
\hline phlox & Phlox spp. \\
\hline photinia, Fraser's & Photinia $\times$ fraseri \\
\hline pigmy date palm & Phoenix roebelinii \\
\hline pin oak & Quercus palustris \\
\hline pincushion flower & Scabiosa cultivars \\
\hline pine & Pinus spp \\
\hline pine, Canary Island & Pinus canariensis \\
\hline pine, Eastern white & Pinus strobus \\
\hline pine, Jack & Pinus banksiana \\
\hline pine, loblolly & Pinus taeda \\
\hline pine, lodgepole & Pinus contorta \\
\hline pine, longleaf & Pinus palustrus \\
\hline pine, Norfolk Island & Araucaria heterophylla \\
\hline pine, sand & Pinus clausa \\
\hline pine, slash & Pinus elliotii \\
\hline pineapple guava & Acca sellowiana \\
\hline pinks & Dianthus plumarius \\
\hline pinwheel jasmine & Jasminum nitidum \\
\hline Preamergence Hebicies for Us in & Ornaments \\
\hline
\end{tabular}

\begin{tabular}{|l|l|}
\hline \multicolumn{2}{|l|}{ Table 5. Common name reference list for ornamental crops. } \\
\hline Common name & Scientific name \\
\hline pittosporum & Pittosporum spp. \\
\hline pittosporum & Pittosporum tobira \\
\hline plane tree, American & Platanus occidentalis \\
\hline plum, natal & $\begin{array}{l}\text { Carissa macrocarpa (synonym C. } \\
\text { grandiflora) }\end{array}$ \\
\hline plumbago & Plumbago auriculata \\
\hline podocarpus & Podocarpus macrophyllus \\
\hline podocarpus & Podocarpus spp. \\
\hline podocarpus, weeping & Podocarpus gracilior \\
\hline portulaca & Portulaca grandiflora \\
\hline pot marigold & Calendula officinalis \\
\hline prairie gentian & Eustoma grandiflorum \\
\hline primrose & Primula spp. \\
\hline primrose jasmine & Jasminum mesnyi \\
\hline princes flower & Tibouchina urvilleana \\
\hline privet & Ligustrum spp. \\
\hline privet, glossy & Ligustrum lucidum \\
\hline privet, Japanese & Ligustrum japonicum \\
\hline privet, variegated Chinese & L. sinense 'Variegated' \\
\hline privet, Vicaryi & Ligustrum $\times$ vicaryi \\
\hline purple anise & Illicium floridanum \\
\hline pyracantha & Pyracantha spp. \\
\hline pyracantha & $\begin{array}{l}\text { Pyracantha. crenatoserrata (synonym } \\
\text { P. fortuneana) }\end{array}$ \\
\hline queen palm & Syagrus romanzoffianum \\
\hline Queen Anne's lace & Ammi majus \\
\hline red buckeye & Aesculus pavia \\
\hline red cedar, Eastern & Juniperus virginiana \\
\hline red cedar, Southern & Juniperus silicicola \\
\hline red maple & Acer rubrum \\
\hline & \\
\hline
\end{tabular}




\begin{tabular}{|l|l|}
\hline \multicolumn{2}{|l|}{ Table 5. Common name reference list for ornamental crops. } \\
\hline Common name & Scientific name \\
\hline red oak & Quercus rubra \\
\hline red-hot poker & Kniphofia uvaria \\
\hline redbud & Cercis canadensis \\
\hline rhaphiolepis & Rhaphiolepis umbellata \\
\hline river birch & Betula nigra \\
\hline rose & Rosa spp. \\
\hline rose, moss & Portulaca grandiflora \\
\hline rose of China & Hibiscus rosa-sinensis \\
\hline rose of Sharon & Hibiscus syriacus \\
\hline rosemary & Rosmarinus officinalis \\
\hline ruscus & Ruscus hypophyllum \\
\hline Russian olive & Elaeagnus angustifolia \\
\hline safflower & Carthamus tinctorius \\
\hline sage & Salvia spp. \\
\hline sage, Texas & Leucophyllum frutescens \\
\hline sago palm & Cycas revoluta \\
\hline salvia & Salvia farinacea \\
\hline sandankwa viburnum & Viburnum suspensum \\
\hline sasanqua camellia & Camellia sasanqua \\
\hline saucer magnolia & Magnolia $\times$ soulangiana \\
\hline Savannah holly & Ilex attenuata 'Savannah' \\
\hline savin juniper & Juniperus sabina \\
\hline Sawara cypress & Chamaecyparis pisifera \\
\hline schefflera & Schefflera arboricola \\
\hline Scotch heather & Calluna vulgaris \\
\hline sea lavender & Limonium perezii \\
\hline sedum & Sedum spp. \\
\hline shasta daisy & Chrysanthemum superbum \\
\hline shining jasmine & Jasminum nitidum \\
\hline shore juniper & Juniperus conferta \\
\hline
\end{tabular}

\begin{tabular}{|l|l|}
\hline \multicolumn{2}{|l|}{ Table 5. Common name reference list for ornamental crops. } \\
\hline Common name & Scientific name \\
\hline shrub, banana & Michelia figo \\
\hline Shumard oak & Quercus shumardii \\
\hline Siberian squill & Scilla siberica \\
\hline silver dollar & Lunaria annua \\
\hline silver dollar tree & Eucalyptus cinerea \\
\hline silver maple & Acer saccharinum \\
\hline slash pine & Pinus elliotii \\
\hline small-leaf jasmine & Trachelospermum asiaticum \\
\hline snapdragon & Antirrhinum majus \\
\hline society garlic & Tulbaghia violacea \\
\hline Southern magnolia & Magnolia grandiflora \\
\hline Southern red cedar & Juniperus silicicola \\
\hline spiderwort & Tradescantia virginiana \\
\hline spiraea, false & Astilbe spp. \\
\hline spiral flag & Costus species \\
\hline spiraea & Spiraea cantoniensis \\
\hline spiraea, Japanese & Spiraea japonica \\
\hline spiraea, Van Houtte & Spiraea $\times$ vanhouttei \\
\hline St. John's wort & Hypericum spp. \\
\hline star anise & Illicium parviflorum \\
\hline star jasmine & Trachelospermum jasminoides \\
\hline statice & Limonium perezii \\
\hline strawberry guava & Psidium littorale var. lucidum \\
\hline strawflower & Xerochrysum bracteatum (see \\
& Helichrysum bracteatum) \\
\hline strawflower & Bracteantha bracteata (see \\
\hline Helichrysum bracteatum) \\
\hline strawflower & Helichrysum bracteatum \\
\hline summer lilac & Acer saccharum \\
\hline & \\
\hline
\end{tabular}

Preemergence Herbicides for Use in Ornamentals

Preemergence Herbicides for Use in Ornamentals 


\begin{tabular}{|l|l|}
\hline \multicolumn{2}{|l|}{ Table 5. Common name reference list for ornamental crops. } \\
\hline Common name & Scientific name \\
\hline sunflower & Helianthus spp. \\
\hline sweet gum & Liquidambar styraciflua \\
\hline sweet tea & Osmanthus fragrans \\
\hline sweet William & Dianthus barbatus \\
\hline sweetpea & Lathyrus odoratus \\
\hline sweetspire & Itea ilicifolia \\
\hline sycamore & Platanus occidentalis \\
\hline tabebuia & Tabebuia caraiba \\
\hline tea, sweet & Osmanthus fragrans \\
\hline tea tree & Leptospermum scoparium \\
\hline ternstroemia & Ternstroemia gymnanthera \\
\hline Texas sage & Leucophyllum frutescens \\
\hline trailing lantana & Lantana sellowiana \\
\hline tree “fern" & Asparagus virgatus \\
\hline tree fern, Australian & Cyathea australis \\
\hline tree, orchid & Bauhinia galpinii \\
\hline tree, tea & Leptospermum scoparium \\
\hline tree, tulip & Liriodendron tulipifera \\
\hline tulip & bulbs, ornamental \\
\hline tulip & Tulipa spp. \\
\hline tulip tree & Liriodendron tulipifera \\
\hline Van Houtte spiraea & Spiraea $\times$ vanhouttei \\
\hline variegated Chinese privet & Ligustrum sinense 'Variegated' \\
\hline verbena & Verbena spp. \\
\hline viburnum & Viburnum spp. \\
\hline viburnum, Japanese & Viburnum japonicum \\
\hline viburnum, sandankwa & Viburnum suspensum \\
\hline Vicaryi privet & Ligustrum $\times$ vicaryi \\
\hline vinca & Vinca spp. \\
\hline voilet, African & Saintpaulia ionantha \\
\hline
\end{tabular}

\begin{tabular}{|l|l|}
\hline \multicolumn{2}{|l|}{ Table 5. Common name reference list for ornamental crops. } \\
\hline Common name & Scientific name \\
\hline Washington fan palm & Washingtonia robusta \\
\hline water oak & Quercus nigra \\
\hline wax myrtle & Myrica cerifera \\
\hline wedelia & Wedelia trilobata \\
\hline weeping bottlebrush & Callistemon viminalis \\
\hline weeping fig & Ficus benjamina \\
\hline weeping podocarpus & Podocarpus gracilior \\
\hline weeping willow & Salix babylonica \\
\hline weigela, old-fashioned & Weigela florida \\
\hline willow & Salix spp. \\
\hline willow oak & Quercus phellos \\
\hline willow, weeping & Salix babylonica \\
\hline wind flower & Anemone coronaria \\
\hline windmill palm & Trqchycarpus fortunei \\
\hline winter jasmine & Jasminum nudiflorum \\
\hline wintercreeper & Euonymus fortunei \\
\hline wintergreen barberry & Berberis julianae \\
\hline wisteria & Wisteria sinensis \\
\hline wort, St. John's & Hypericum spp. \\
\hline xylosma & Xylosma congestum \\
\hline yarrow & Achillea spp. \\
\hline yaupon holly & Ilex vomitoria \\
\hline yucca, Adam's needle & Yucca filamentosa \\
\hline zinnia & Zinnia elegan \\
\hline
\end{tabular}

